\title{
Ces experts qui venaient du froid Comment des Canadiens deviennent experts en développement de l'Afrique subsaharienne
}

\author{
par \\ Laurent Paradis-Charette
}

Département des sciences sociales

Mémoire présenté à l'Université du Québec en Outaouais comme exigence partielle du programme de maîtrise ès arts en développement territorial profil développement international

Février 2017

(C) Laurent Paradis-Charette, 2017 


\section{REMERCIEMENTS}

J'aimerais tout d'abord remercier gracieusement Audrey, Carl, Frank, Gérald, Leonard, Marie et Maurice (noms fictifs) soit les sept personnes anonymes qui ont gentiment et courageusement accepté de participer à cette recherche. Sans leur ouverture, leur temps et leur disponibilité, cette recherche n'aurait pas eu lieu.

J'aimerais également remercier mon directeur Dimitri della Faille. Les nombreuses expériences de recherche, de rédaction et de présentation auxquelles tu m'as initié m'ont permis d'activement développer mes compétences. Ensemble nous avons énormément appris, ce qui fera de nous de meilleurs chercheurs et de meilleurs enseignants.

Je remercie aussi mes réviseuses, Marjolaine Villeneuve et Valérie La France-Moreau pour leurs commentaires et révisions linguistiques indispensables. Stéphanie Demers, je n'aurais jamais terminé sans ton soutien indéfectible, tes encouragements incessants, ton optimisme, ton engagement débordant auprès de moi et de mon travail, tes conseils et ton temps pour un étudiant qui n'est même pas dans ton département ou tout simplement sous ta responsabilité. Pour te paraphraser : « je suis sans mot. »

Je remercie également Thibault Martin, qui a su me transmettre son amour de la recherche. Par delà de tes compétences, c'est surtout ta disponibilité, ton ouverture, ton humilité, ta technique unique de gestion de tâche par boîte courriel et ton humour sans bornes qui m'ont aidé à retrouver l'humanité 
et le plaisir dans le travail universitaire.

Merci, aux collègues du département, De Anarchist Mob et tous ses collaborateur.e.s : «Pra, Pra ». Parmi tous ces gens, trois personnes méritent une ligne, tout d'abord Shawn Goodman et Antonin Bourret, camarades de lutte et de cynisme libérateur, sans oublier Valérie La France-Moreau qui, bien qu'elle fasse partie de la première catégorie, mérite qu'on souligne sa ténacité hors du commun. Tu es un exemple de détermination et tu m'as énormément appris et inspiré à terminer ce mémoire. Bien sûr, ces remerciements se doivent d'inclure les travailleuses de l'ombre du département de sciences sociales, Céline Morisset et Nancy Béliveau. Merci pour votre travail acharné et votre bonne humeur. J'espère que vous savourerez la lecture de ces remerciements autant que ma jasette!

Par ailleurs, j'aimerais remercier Jean, Denis, Luc, Yves et André qui ont su expertement repousser les limites de la médiocrité en matière de gestion universitaire. Votre imperméabilité de gestionnaire, votre insuffisance vis-à-vis la mission universitaire d'accessibilité, votre infantilisation des étudiants.e.s, votre misogynie et votre inaptitude à agir en dehors des cadres ridicules dans lesquels vous vous êtes encarcanés m'ont aidé à comprendre comment on pouvait subir une bureaucratie. Grâce à tout cela, je me suis fait un tas de camarades qui comme comme moi ont réalisé qu'on ne vous doit rien!

J'aimerais remercier mes beaux-parents Michel et Josée qui nous ont épaulés moi et Marie-Pier dans cette aventure qui n'en finissait plus. Vous nous avez donné le souffle et les petites détentes nécessaires pour passer au travers de ce marathon. 
Je tiens aussi à remercier mon père de m'avoir transmis l'esprit critique et scientifique. Je tiens à remercier ma mère et ma soeur de m'avoir transmis cet intérêt pour les humains et l'empathie. Désolé papa, il faudra que tu l'admettes un jour, maman et Nini ont raison : les gens et les arts sont beaucoup plus intéressants que les cochons et les poulets. J'aimerais remercier mes enfants Dimitri, Danahé et Éthelle pour leur soutien, leur patience, leurs fous rires, leurs encouragements, leur amour indéfectible et les œuvres qui ensoleillent mon bureau.

En dernier lieu, j'aimerais remercier ma meilleure amie, amoureuse et compagne pour son soutien de tous les instants. Marie-Pier, tu es coauteure dans mon cœur. Tu es la femme la plus extraordinaire du monde. Je t'aime $>\infty$. 
Ce mémoire a été entièrement produit à l'aide des logiciels libres $\underline{\text { Zotero, }}$ Docear et LibreOffice. 


\title{
RÉSUMÉ
}

Chaque année, des centaines d'experts canadiens offrent leurs avis, leurs compétences, leurs connaissances ou leurs savoirs-faire, afin de mettre en place, de suivre et d'évaluer des projets et des programmes destinés à développer des communautés d'Afrique subsaharienne. Le Canada étant un pays nordique situé au minimum à plus de 5000 kilomètres des côtes africaines, il devient logique de se demander comment des étrangers venus de si loin sont devenus experts du développement des

communautés africaines. À partir de la théorie du champ de Bourdieu, l'analyse des entretiens menés dans le cadre de cette recherche nous indique que, pour devenir expert, il faut être en mesure d'accumuler, de mobiliser, d'échanger ou de transformer des capitaux technologiques, organisationnels, sociaux et d'expertises spécifiques à l'occident. En ce sens, le champ de l'expertise en développement international se trouve en lutte constante pour sauvegarder son autonomie vis-à-vis des champs qui dominent la société occidentale, notamment le champ économique et le champ du pouvoir.

\section{Mots-clés}

Expertise, expert, savoir-faire, développement international, coopération internationale.

\begin{abstract}
Each year, hundreds of Canadian experts offer their opinions, their skills, their knowledge or knowhow to implement, monitor and evaluate projects and programs aimed at developing communities in the Sub-Saharan African region. Canada is a northern country located roughly $5000 \mathrm{~km}$ from the nearest African shore, therefore it becomes logical to ask how foreigners can become experts in the development of African communities. Using Bourdieu's field theory, we analyze the interviews gathered in this research to show that experts must be able to accumulate, mobilize, trade or transform technological, organizational, social and expertise capitals specific to the western world. Because of that, the field of expertise in international development is in a constant struggle to reaffirm its autonomy next to the dominant fields of the western world notably the political and economical fields.
\end{abstract}

\section{Keywords}

Expertise, Expert, Know-how, International development, International cooperation. 


\section{TABLE DES MATIÈRES}

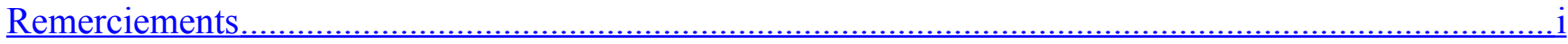

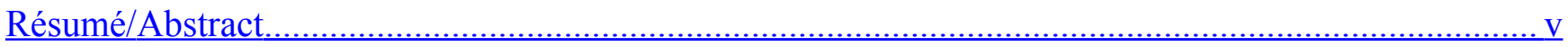

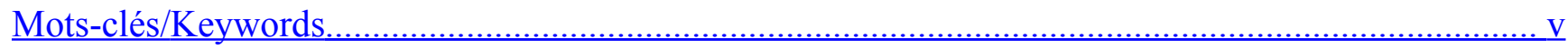

Table des matières....................................................................................................................... vi

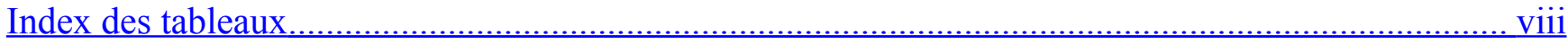

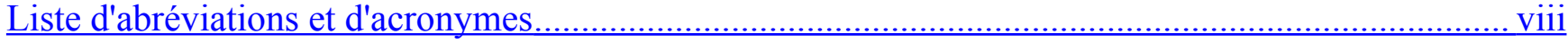

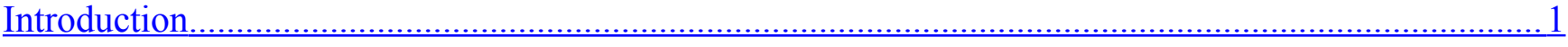

Chapitre I - Problématique et contexte .................................................................................................... 4

I.1 - Mise en contexte : L'expertise et sa relation avec le développement international...........................4

I.2 - L'expertise une préoccupation sociale, politique et théorique ……………….............................

I.3 - Questions de recherche ......................................................................................................... 19

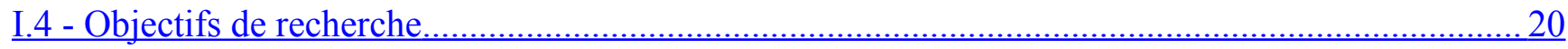

Chapitre II - Cadre Théorique - La Théorie du Champ..........................................................................23

II.1 - Émergence d'une théorie du champ. ………………..............................................................24

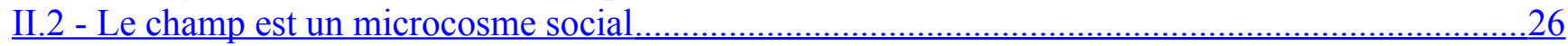

II.3 - Revue critique ................................................................................................................ 34

II.4 - Ce qu'on en retient : le Champ de l'expertise en développement international..............................36

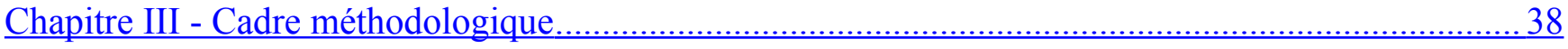

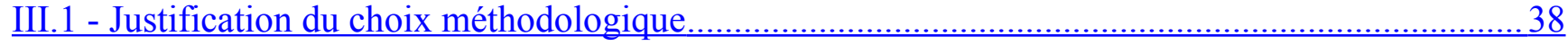

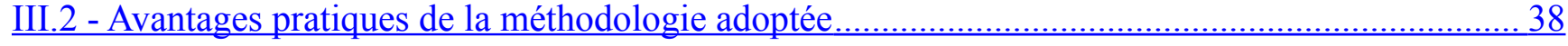

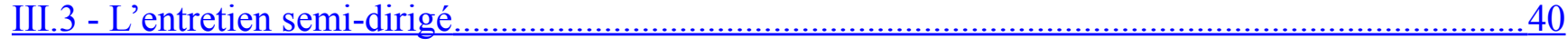

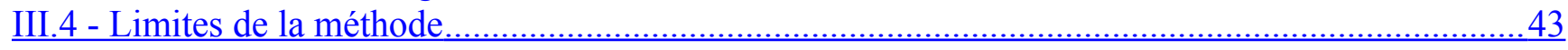

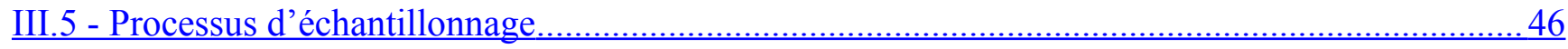

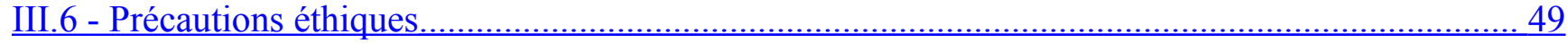

III.7 - Description de l'échantillon.............................................................................................. 51

Chapitre IV - Présentation des résultats de l'enquête .......................................................................57

IV.1 - Les impératifs de l'expertise en développement international ........................................................ 57

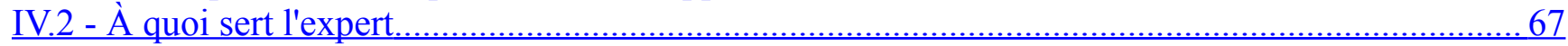

IV.3 - Ambiance de pratique de l'expertise en développement international ........................................ 73

IV.4 - Les situations d'expertise ................................................................................................. 84

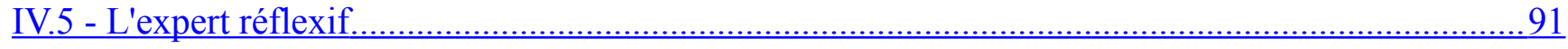

Chapitre V - Analyse - Le champ de l'expertise en développement international................................115

V.1 - Structure des capitaux spécifiques.........................................................................................115

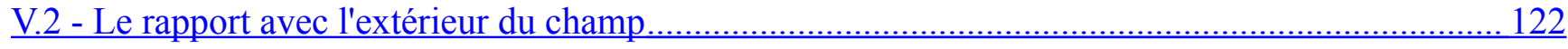

V.3 - Le champ de l'expertise comme microcosme social................................................................ 126

V.4 - Résultats de l'analyse .................................................................................................. 134

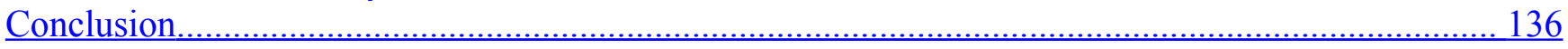

Le champ de l'expertise : changement de régime et enjeux de lutte ................................................. 137

Limites de la recherche ....................................................................................................... 144

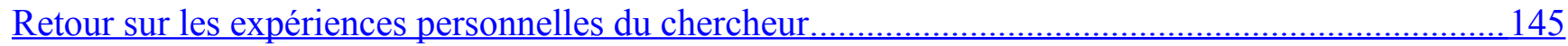

Bibliographie ..................................................................................................................... 148

Annexe 1-Copie du texte du premier contact téléphonique ............................................................. 162

Annexe 2-Copie du texte du premier contact postal ou par courrier électronique ...............................164

Annexe 3 - Guide d'entretien..................................................................................................... 166

Annexe 4-Formulaire de consentement ........................................................................................ 168 


\section{INDEX DES TABLEAUX}

Tableau A - Frontières de champ et composition d'un espace social...............................................22

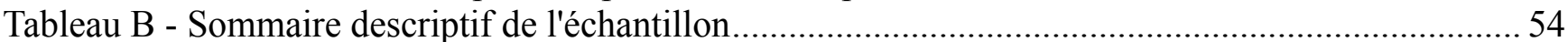

\section{LISTE D'ABRÉVIATIONS ET D'ACRONYMES}

ACDI : Agence canadienne de développement international

AQOCI : Association québécoise des organismes de Coopération Internationale

$\mathrm{BM}$ : Banque mondiale

Banco del Sur : Banque du Sud

CCCI : Conseil canadien pour la coopération internationale

FMI : Fonds monétaire international

MAÉCD : Ministère des Affaires étrangères du Commerce et du Développement

OCDE : Organisation de coopération et de développement économiques

OI : Organisations internationales

ONG : Organisme nongouvernementaux

ONU : Organisation des Nations Unies

OXFAM : Contraction transformée en nom propre issu du nom original « Oxford Committee for Relief Famine »

PLC : Parti libéral du Canada

PNUD : Programme des Nations Unies pour le développement

SACO : Service d'assistance canadienne aux organismes 


\section{INTRODUCTION}

Depuis plus de 66 ans, le Canada possède un service d'aide au développement destiné à aider les communautés de certains pays à se développer. D'abord fourni par le Bureau d'aide extérieur, puis par l'Agence canadienne de développement international (ACDI), l'aide au développement est aujourd'hui offerte par le ministère des affaires étrangères, du commerce et du développement (MAÉCD). Au cours de ces soixante-six ans, des générations d'experts se sont succédé afin d'offrir leurs avis, leurs compétences ou encore leurs savoirs afin de mettre en place, de suivre et d'évaluer des projets et des programmes destinés à développer des communautés d'Afrique subsaharienne. En effet, le Canada, à l'instar de nombreux pays occidentaux et riches, a envoyé de nombreux savants, techniciens et spécialistes de l'aide au développement. Le Canada étant un pays nordique situé au minimum à plus de 5000 kilomètres des côtes africaines, il devient logique de se demander comment des étrangers venus de si loin sont devenus experts du développement des communautés africaines.

Dans ce mémoire, nous nous proposons d'examiner comment des Canadiens sont devenus experts

en développement international. À partir de la rencontre de sept experts canadiens, nous souhaitons déterminer comment des Canadiens deviennent experts en développement de l'Afrique subsaharienne.

Dans le premier chapitre, nous avons ancré la problématique dans son contexte et examiné les préoccupations sociales, politiques et théoriques qu'elle soulève. Ensuite nous avons cerné plus 
précisément les questions et des objectifs de recherche spécifiques qui nous permettront de répondre au mieux à nos questionnements.

Au chapitre II, nous avons mobilisé un cadre d'analyse inspiré de l'œuvre que Pierre Bourdieu a développée autour du concept de champ. Grâce à ce cadre, nous serons en mesure d'identifier non seulement les frontières et les structures que ces personnes doivent surmonter pour devenir expertes, mais aussi les règles avec lesquelles elles doivent composer et les capitaux particuliers qui leur permettent de devenir expertes en développement international.

Au chapitre III, nous explicitons la démarche méthodologique choisie dans le but d'accumuler une quantité de données suffisante à l'analyse. Pour ce faire nous avons choisi de procéder à des entretiens dirigés et décrit la procédure utilisée tout en restant conscient de la portée et des limites des outils choisis. De même, nous avons procédé à un examen similaire pour ce qui est de l'échantillon choisi. En dernier lieu, nous avons fait un examen des dilemmes éthiques posés par nos choix et fait état des précautions prises pour en limiter les effets négatifs.

$\mathrm{Au}$ chapitre IV, nous présentons les résultats issus des entretiens semi-dirigés que nous avons menés selon les principales thématiques qu'ont abordées les personnes rencontrées, soit (1) les impératifs de l'expertise, (2) son utilité, (3) l'ambiance dans laquelle les experts estiment pratiquer et (4) les situations dans lesquelles on fait appel aux experts.

$\mathrm{Au}$ chapitre $\mathrm{V}$, nous analysons les données ainsi produites. Pour ce faire, nous établissons des liens entre la problématique que nous avons élaborée, les éléments du cadre théorique mobilisé et les propos recueillis auprès des gens que nous avons rencontrés dans le cadre des entretiens semi-dirigés. 
Cette analyse nous aidera à mieux comprendre comment des Canadiens deviennent experts en développement international.

Enfin, la conclusion procède à un résumé des constats issus de ce mémoire et des enseignements que nous pouvons en tirer. 


\section{ChaPITRe I - ProblématiQue et CONTeXte}

Dans ce chapitre, nous présenterons d'abord une mise en contexte de notre problématique de recherche. Pour ce faire nous avons procédé à une revue critique de la littérature scientifique afin d'identifier les connaissances manquantes relatives à l'expertise en développement international. Par la suite, nous exposerons en quoi elle constitue un enjeu politique, social et théorique. Pour terminer, nous présenterons les questions et les objectifs de la recherche qui nous permettront d'améliorer l'état des connaissances actuelles de celle-ci.

\section{I.1 - Mise en contexte : L'expertise et sa relation avec le développement international}

L'expert en développement international agit depuis longtemps dans le monde des institutions du développement sous diverses formes et divers mandats. Il provient de différentes disciplines, principalement des sciences sociales et de la nature. Malgré la diversité d'activité, d'orientation et de disciplines de l'expertise, certains remettent en question l'organisation de l'encadrement du développement à travers le prisme de l'expertise. Dès 1965, le sociologue Jacques Berque établissait les bases d'une réflexion critique sur l'expertise technique dans le contexte de la décolonisation de l'Afrique (Berque 1965). Alors qu'il réfléchissait au fonctionnement de l'assistance technique, Berque tâchait de lier expertise, politique étrangère et impérialisme, tout en faisant preuve de certaines ruptures avec le régime colonial. Encore aujourd'hui, l'expertise technique reste au centre de l'action de développement, mais sa pratique échoue (Latouche 1986; Rist 2007; Easterly 2013). Du triomphe de la 
modernisation, en passant par le « consensus de Washington $»^{1}$ et les Objectifs du millénaire pour le développement (OMD), les experts continuent d'avoir un rôle attesté dans la mise en place des objectifs du développement et du choix des moyens pour les atteindre. Malgré cet usage de l'expertise censée être un gage de réussite, une majorité d'acteurs du milieu du développement international et de chercheurs en développement international s'entendent pour dire que beaucoup de difficultés freinent l'atteinte des objectifs fixés par les politiques de développement, voir, que le développement international est un échec (Escobar 1995; Cassen 1994; Barnett et Finnemore 2003; Ovaska 2003; Mosse 2005; North 2005; Sachs 2005; Rist 2007; Williamson 2009). La mise en place des programmes par les institutions internationales ne mènerait pas à une amélioration des niveaux de santé, d'éducation et de nourriture, comparativement à ceux promis par les institutions. Si certains atteignent leurs objectifs par hasard (Ferguson 1994; Swidler et Watkins 2009), d'autres aboutissent à des effets contraires (Andersen 2000). Or, si le développement ne semble pas remplir les objectifs ciblés ou encore ne pas être contrôlé par ceux qui décident de son application, il devient important de se pencher sur les mécanismes du développement qui permettraient de comprendre comment sont prises les décisions dans ce domaine pour mener systématiquement à ce constat.

Le discours d'inauguration du président étasunien Harry S. Truman en 1949 est célèbre. ${ }^{2}$ On dit qu'il consacre le début de l'ère du développement. Depuis ce discours, l'idée de sous-développement et la nécessité inhérente de développer les nations dites plus pauvres occupent des agences étatiques, des organismes non gouvernementaux et des organisations internationales dans une lutte pour extraire le

1 Voir section I.2.a (p.8)

2 « [...] Il nous faut lancer un nouveau programme qui soit audacieux et qui mette les avantages de notre avance scientifique et de notre progrès industriel au service de l'amélioration et de la croissance des régions sous-développées. [...] Leur vie économique est primitive et stationnaire. Leur pauvreté constitue un handicap et une menace, tant pour eux que pour les régions les plus prospères. [...] Je crois que nous devrions mettre à la disposition des peuples pacifiques les avantages de notre réserve de connaissances techniques afin de les aider à réaliser la vie meilleure à laquelle ils aspirent. Et, en collaboration avec d'autres nations, nous devrions encourager l'investissement de capitaux dans les régions où le développement fait défaut. [...] Une production plus grande est la clef de la prospérité et de la paix. "Discours d'investiture d'Harry Truman, président des États-Unis, le 20 janvier 1949 cité dans (Rist, 2007) 
monde « pauvre» de la nécessité par le biais d'une assistance technique et financière (Rist 2007). Depuis les premiers constats d'échecs de ce modèle de développement vers la fin des années 1960, une course entre « développeurs » pour « s'ajuster », « revoir ses pratiques » « s'adapter aux nouvelles normes » et « atteindre nos objectifs » donne naissance à une panoplie d'expressions pour faire référence à un développement « bon » et « vrai » par rapport à son essence (Legouté 2001).

Le recours à l'expertise et aux experts est ainsi devenu une pratique constante non seulement pour réduire la pauvreté afin d'améliorer le sort des moins nantis, mais aussi nécessaire pour démêler, à travers l'évaluation, le « vrai » développement du développement « fantasque ». Ainsi, certains auteurs regrettent que le manque d'accès à la connaissance ait comme effet la répétition constante des mêmes erreurs (Easterly 2006; Djankov, Montalvo et Reynal-Querol 2008; Doucouliagos et Paldam 2009). Pour d'autres, la pauvreté perdure parce que les bénéficiaires des politiques de développement ne sont pas en mesure de mobiliser adéquatement la connaissance afin de donner un point de vue multidimensionnel sur leur situation sociale et économique (Collier 2006; Banerjee et Duflo 2011). C'est à ce titre que des organismes nongouvernementaux (ONG), des agences étatiques, des organisations internationales $^{3}(\mathrm{OI})$, des entreprises privées ou encore des scientifiques souhaitent que l'expertise occupe une place plus importante dans les processus de prise de décision en développement international afin de mettre fin au cycle de « crises » qui l'affecte depuis son institutionnalisation.

Suivant cette pratique, l'utilisation de l'expertise est devenue inhérente à l'idée de faire du développement. Ainsi, l'arrivée de l'« expert» en développement devient alors une chose courante et habituelle pour qui participe à une activité de développement dans les pays dits « en voie de

3 Notamment, l'Organisation des Nations Unies (ONU), le Programme des Nations Unies pour le Développement (PNUD), la Banque mondiale (BM), le Fond Monétaire International (FMI), la Banque du Sud (Banco del sur), pour ne nommer que celles-là. 
développement », particulièrement en Afrique subsaharienne. L'expertise s'est spécialisée depuis les premières mesures de développement pour se présenter sous différentes catégories ou formes et dans

des contextes variés. À tel point, qu'au cours des dernières décennies, on a assisté à l'émergence des consultants comme une catégorie d'experts en développement international à part entière (Stirrat 2000; Subramaniam 2007), plus particulièrement avec le retrait progressif du financement du secteur public de l'aide au développement peu à peu remplacé par des donateurs privés (Li 2005; Li 2007). Les experts consultants sont rattachés à une institution et surtout à des fonds de financement de projet de développement pour une période déterminée, sur un sujet précis à propos duquel ils doivent apporter une expertise qui servira à mettre en place les volontés de son employeur. Souvent constituée d'experts contractuels, cette catégorie d'experts est par la suite libre d'offrir son expertise à tous. C'est principalement à cette catégorie d'experts qu'on demande de « conceptualiser », « orienter », « évaluer » des « besoins », « recommander» des «projets », des «mesures» de développement adaptés aux communautés visées par un projet de développement.

\section{I.2 - L'expertise une préoccupation sociale, politique et théorique}

Comme nous venons de le démontrer, depuis la décolonisation, l'expertise fait l'objet de profond débat tant dans la société et au sein des sciences sociales et humaines que dans le monde du développement international. En effet, l'apport d'expertise est souvent considéré par les récipiendaires de l'aide au développement comme l'un des principaux moyens utilisés pour aider au développement d'une communauté. Cet apport peut consister en l'envoi de personnes avec des compétences reconnues chargées d'évaluer ou de mettre en place une nouvelle pratique de développement. Par exemple, un économiste et un anthropologue peuvent être envoyés pour évaluer un projet de transformation de balle de riz au Burkina Faso et déterminer quels éléments peuvent être généralisables et transposables à 
d'autres communautés ou pays.

Sur le plan social et politique, les transformations actuelles dans les politiques canadiennes de développement international font l'objet de sérieux débats à propos de la provenance, de la forme, du financement et des motifs invoqués pour justifier de l'aide appelé à mobiliser l'expertise en développement international (Mackrael 2012; Samantha Nutt 2012; Brender 2012; Brown 2012; Kindornay et al. 2013; MiningWatch Canada 2013; Conseil canadien pour la coopération internationale 2014; Gilmore 2014). Nous entendons démontrer dans les paragraphes suivants comment l'expertise constitue un enjeu théorique en sciences sociales et en développement international constituant parallèlement un enjeu politique et social pour la société canadienne ainsi qu'un enjeu pour les récipiendaires de l'aide canadienne au développement.

\section{I.2.a - L'expertise et le néolibéralisme}

Comme nous allons tenter de le démontrer dans cette section, l'expertise contemporaine tient sa forme en grande partie de son inscription dans une manière particulière d'envisager l'économie, la société et la politique. Le néolibéralisme est cette manière dominante de penser les rapports sociaux. Le néolibéralisme a une importante prégnance au sein des institutions internationales et nationales. Pour les raisons que nous allons exposer, cette manière de penser les rapports sociaux consacre le recours à l'expertise.

Sur le plan historique, le terme de néolibéralisme voit le jour afin de désigner un courant de théories économiques, morales et sociales inspiré par les travaux d'universitaires allemands des années 1930 et 1940 (Boas et Gans-Morse 2009). L'usage de ce terme décline dans les années suivantes, puis 
selon certains auteurs celui-ci resurgit à partir des années 1970 -1980 pour désigner péjorativement les idées et politiques économiques associées à des universitaires ou intellectuels tels que Frederick von Hayek, Ludwig von Mises, Milton Friedman et Ayn Rand (Boas et Gans-Morse 2009; Brenner, Peck et Theodore 2010). On associe alors ces idées à des politiques publiques que les critiques qualifient d'idéologie mise en place par Augusto Pinochet au Chili, Margaret Thatcher au Royaume-Uni ou Ronald Reagan aux États-Unis d'Amérique (Boas et Gans-Morse 2009) et plus tard pour désigner les politiques de développement prescrites par les programmes d'ajustement structurels mis en place par les institutions dîtes du « consensus de Washington » (Williamson 1990; Peck et Tickell 2002).

Le concept est devenu un référent pour des politiques publiques qui se rapportent à cette idéologie, à une tendance dans la transformation des institutions, à une forme émergente de subjectivité et à une réflexion sur le réalignement idéologique ou à une combinaison de ces idées (Brenner, Peck et Theodore 2010, 183). Dans cette optique, au tournant des années 2000, le terme fait référence à un projet idéologique basé sur une conceptualisation fondamentaliste de l'économie de marché qui favorise la réduction du contrôle de l'État, une moralité autoritaire avec une famille idéalisée en son centre, des principes de libremarché internationaux (appliqués de façon très inconsistante) et une intolérance sévère des mouvements syndicaux (Bourdieu et Wacquant 1998; Brenner et Theodore 2002). Le concept fait l'objet d'une utilisation sans cesse croissante depuis les années 1980 (Boas et Gans-Morse 2009) et nourrit un important débat théorique et conceptuel qui fait fructifier plusieurs courants.

Sur le plan conceptuel, un examen de la littérature actuelle nous indique que plusieurs points de vue s'affrontent. Certains auteurs conceptualisent le néolibéralisme comme une idéologie hégémonique (Jessop 2002; Hoffman, DeHart et Collier 2006; Richland 2009). D'autres au contraire s'inspirent de la 
notion de «gouvernementalité » (Foucault 2004) de Michel Foucault et conceptualisent le néolibéralisme comme une rationalité normative, sans véritable idéologie et instable (Boltanski et Chiapello 1999; Laval et Dardot 2009) qui s'applique à toutes les sphères de la société (Larner 2000; Ferguson 2006; Ong 2007). D'autres le considèrent comme un concept qui se résume à la critique négative unilatérale du capitalisme (Ferguson 2009; Collier 2012). Entre ces deux pôles, d'autres préfèrent s'inscrire à mi-chemin de ces deux conceptualisations. Ces auteurs entrevoient ainsi le néolibéralisme comme un projet idéologique construit par un corps institutionnel (l'État) qui comporte une spatialité distincte, c'est-à-dire que d'un lieu, d'un contexte, d'une culture ou d'un espace à l'autre où le néolibéralisme sera pratiqué, ce dernier prendra diverses formes, se manifestera de manières dissemblables et se matérialisera par l'insertion de mécanismes qui favoriseront une logique économique de la répartition des capitaux (Brenner, Peck et Theodore 2010; Hilgers 2012; Wacquant 2012). Cette logique produit des conditions particulières donnant lieu au renforcement des fonctions étatiques de contrôle et de coercition, où l'État a la particularité de déléguer invariablement sa mission à des compagnies privées ou encore la privatise informellement (Hilgers 2012). Pour d'autres encore, ces conceptualisations sont trop abstraites, car elles ne parviennent pas à systématiser les mécanismes d'interaction avec les éléments comme la culture des groupes sociaux (Kingfisher et Maskovsky 2008). Pour les besoins de ce mémoire, nous retiendrons la définition du néolibéralisme issue des travaux d'Hilgers.

À ce titre, étudier l'expertise en développement international au Canada, c'est s'intéresser au néolibéralisme; car dans l'aide canadienne, l'exécution est déléguée principalement à des sous-traitants chargés de la mise en œuvre de projets et programmes de développement. Les futurs «Agents d'exécution - Partenaires $-»^{4}$ sont des ONG, des associations, des compagnies privées, des

4 Pour utiliser le terme mis de l'avant par l'ACDI et que le MAECD continue d'utiliser. (MAÉCD 2015) 
établissements d'enseignement, des instituts, des ministères, etc., désirant participer aux programmes d'aide au développement de l'État canadien, appelés à soumettre une offre que celui-ci choisira ou non. À tout moment dans ce processus, l'État canadien contrôle le financement des projets et exige de ces acteurs privés qu'ils rendent des comptes, des évaluations prenant la forme de rapports périodiques et ayant pour effet de privatiser informellement une vaste majorité des activités canadiennes d'aide au développement.

Maintenant que nous avons présenté le contexte général qui donne forme, selon nous, aux rapports économiques, politiques et sociaux orientant les politiques de développement et le recours aux experts, nous allons expliquer pourquoi l'expertise est un enjeu de préoccupations théoriques pour les sciences sociales ainsi qu'un enjeu social et politique.

\section{I.2.b - Un enjeu de préoccupations théoriques des sciences sociales}

L'étude de l'expertise est un champ des sciences sociales et humaines qui est investi par différentes disciplines. On y retrouve, par exemple, des politologues, des sociologues, des anthropologues, des historiens et aussi des philosophes. Les domaines de recherche en étude de l'expertise sont, eux aussi, très variés. On examine ainsi des implications de l'expertise dans des domaines aussi diversifiés que la gestion des ressources naturelles et de l'environnement, les organisations bureaucratiques, le domaine économique et les finances, le développement social et économique, le domaine criminel et juridique, la gouvernance urbaine, les sciences et technologies, la psychologie de l'apprentissage ainsi que l'étude du langage et des messages médiatiques. Comme cette diversité de disciplines et de domaines de recherche peut le suggérer, l'expertise est envisagée depuis une diversité d'approches, de points de vue et de conception, ce qui rend difficile toute tentative de 
définition de l'expertise à partir d'une synthèse de ces mêmes travaux. Ainsi, encore aujourd'hui, la communauté scientifique continue d'appeler à l'étude de l'expertise et des experts afin de mettre en lumière la façon dont les experts influencent ou sont influencés dans les processus de décisions (Dumoulin et al. 2005; Berrebi-Hoffmann et Lallement 2009; Boltanski 2009; Barbier et al. 2013).

Nous sommes d'avis que l'étude de l'expertise et des experts en développement international est un excellent sujet dont l'étude permet de mettre en lumière les conditions de production et de reproduction des discours à propos du développement. Dans un contexte où les dépenses en matière d'aide au développement sont en croissance depuis plus de 15 ans (OCDE 2015), ces discours ont un impact croissant sur la façon dont sont abordées les problématiques de développement social, économique, environnemental et politique. Les discours issus de l'expertise posent souvent les limites de ce qui devient une connaissance acceptable et mobilisable pour faire face à un problème. Les experts en développement influencés par ceux-ci sont appelés eux même à problématiser les situations rencontrées, à répertorier ce qu'ils sont appelés à qualifier de "problème » de développement ou non. En ce sens, l'étude de l'expertise et des experts permet donc d'identifier les mécanismes faisant en sorte que certaines problématisations sont valorisées plutôt que d'autres en développement international.

De plus, en mettant en lumière ces mécanismes, nous croyons être en mesure d'offrir aux experts une occasion de prendre du recul sur leur pratique. Notre travail devrait leur permettre de tenir compte de la relation entre leurs situations personnelles et professionnelles dans leurs analyses et problématisations du développement. 


\section{I.2.c - Un enjeu social du développement international}

En matière de développement international, la majorité des travaux sur l'utilisation des experts et de l'expertise se concentrent sur les institutions et organismes internationaux de développement comme la Banque mondiale, l'Organisation des Nations Unies et le Fonds monétaire international (Goulet 1980; George et Sabelli 1994; Wade 1996; Bøås et McNeill 2003; Wilson 2006; Broad 2006; 2007; St. Clair 2006; Easterly 2013). Si la présence récurrente des experts dans le paysage du développement nous semble normale, leurs échecs le semblent tout autant : plusieurs programmes, projets et politiques de développement continuent à échouer ou à réussir par hasard et pourtant, de multiples institutions continuent à leur faire confiance. L'expert et l'expertise en développement international possèdent des caractéristiques qui font que l'on retourne constamment les consulter. Pour cette raison, certaines personnes choisissent une carrière professionnelle dans ce champ. Les conditions dans lesquelles ces personnes agissent varient grandement d'un expert à l'autre. Face à ces constats, plusieurs discours sur l'expertise trouvent écho dans la littérature scientifique. Ainsi, certains auteurs en arrivent à la conclusion que les institutions font appel à l'expertise et l'utilisent pour légitimer leurs propres fins (Le Naëlou 1994; Brint 1996; Scott 1998; Mehta 2001; Wilks et Lefrançois 2002; Cornwall et Brock 2005; Kothari 2005; St. Clair 2006; Broad 2007), alors que d'autres perçoivent que l'expertise est responsable de cette situation parce qu'elle s'efforce de maintenir un monopole sur la connaissance à destination du développement (Rayner 2003a; Rayner 2003b; Wilson 2006; Owakah et Aswani 2009).

Pour d'autres auteurs, l'expertise n'est que le simple médium qui interprète les décisions de décideurs ou encore celle de la société civile pour les décideurs (Kennedy 2005; Prevost 2005; Reynolds 2008; Bourguignon 2012), alors que certains estiment qu'elle est un agent de normativité du développement international par l'entremise de multiples réseaux qui prennent forme à travers les 
institutions telles que les ONG, les OI et les firmes de consultants ou encore qu'elle n'est qu'une simple diversion (Carapico 2002; Kennedy 2005; Atlani-Duault 2006; Green 2012; Ziai 2013). Pour d'autres encore, bien que l'on traite publiquement de ses réussites et de ses échecs, le monde du développement lui-même est un monde fermé, quasiment hermétique et hautement ritualisé dans le but de maintenir son emprise sur les sociétés visées (Andersen 2000; Mitchell 2002; Griffith 2003; Niane 2011). C'est un monde où règnent les relations de travail basées sur des contrats à durée limitée, sur des clauses de confidentialités, des cercles restreints de décideurs et où la critique est contrôlée par la peur éprouvée par les experts de perdre leur emploi ou de manquer de futures opportunités (Sogge 2003; Griffith 2003).

Bien que les avis et les perspectives sur l'expertise en développement international divergent, on peut noter que selon les contextes, ces positions ont en commun d'évoquer l'importance de l'effet de cadrage de l'expertise qui établit ce qui est acceptable comme pratiques discursives de l'expertise en développement à tous les niveaux (Della Faille, Lafrance-Moreau et Paradis-Charette 2016). La littérature expose ainsi un contexte tendu dans le milieu du développement où les dirigeants (Broad 2006), les experts (Teichman 1997) et les autres acteurs du développement (Reynolds 2008) s'accusent mutuellement d'imposer un cadre aux pratiques et une hiérarchisation aux types d'expertise, empêchant ainsi tout échange et intégration de connaissances pour le développement. Alors que telles recherches s'intéressent principalement au contexte de la réalité des institutions internationales plutôt qu'à celui de la réalité des experts sur le terrain, il nous apparaît nécessaire d'exposer le point de vue de ces experts. Au cours des dernières années, quelques recherches se sont penchées sur certaines réalités spécifiques. Certains l'ont approché en décrivant « ce que fait » l'expert (Stirrat 2000), d'autres en abordant ce qu'il fait sur le «terrain » des grandes institutions (Griffith 2003; Niane 2011) ou encore à propos de l'influence de l'expertise sur un projet particulier (Subramaniam 2007), mais, les recherches qui 
souhaitent traiter de ces différentes réalités en une même analyse sont peu nombreuses.

\section{I.2.d - Un enjeu social pour l'aide canadienne au développement}

Les experts canadiens qui souhaitent participer à l'édification d'un monde meilleur n'échappent pas à cette réalité. En effet, le monde du développement, sa structure et son rapport avec les pays dits « en voie de développement » traversent une profonde mutation (Harman et Williams 2014) à laquelle l'univers et les institutions canadiennes du développement international n'échappent pas (Essex 2012). Parallèlement, l'idéologie néolibérale qui est dominante dans le domaine du développement international depuis les années 1980 est « en crise » perpétuelle. Elle tente de se refaire une beauté et d'être plus « humaine » par des ajustements constants (Gore 2000; Peck et Tickell 2002; Sandbrook 2007; Brenner, Peck et Theodore 2010; Duménil et Lévy 2011; Hilgers 2012; Wacquant 2012). Il s'agit d'une tendance lourde à laquelle le Canada n'échappe pas, à l'instar de plusieurs pays tels la Norvège, le Danemark et le Royaume-Uni (ACDI 2002).

Ainsi, le Canada multiplie les actions destinées à accroître «l'efficacité » de son aide au développement, et se donne pour objectif de la rationaliser, de concentrer ses activités dans des secteurs et des pays précis (ACDI 2002). Par ce processus, le Canada s'est efforcé de « délier » son aide au développement à travers des «appels d'offres internationaux assujettis à des règles d'approvisionnement libéralisées » (CCCI 2001) dans le but de la rendre plus efficace (OCDE 2001). On a ainsi permis aux entreprises commerciales privées et aux firmes de consultants de soumissionner pour les appels d'offres de l'ACDI afin de mettre en place des projets dans les pays en développement. Par cette logique, le gouvernement canadien rend disponibles les deniers publics de l'aide au développement à la logique économique de l'entreprise privée alors qu'en principe, ils étaient 
auparavant attribués dans une logique «désintéressée » à des Organismes sans but lucratif. Le gouvernement conservateur, entré en fonction suite aux élections de 2006, poursuit sur cette lancée et procède à la réduction du nombre de pays bénéficiaires de l'aide, au déploiement de plus de personnel sur le terrain et exige une prodution accrue d'évaluations dites « indépendantes » et de rapports sur l'aide au développement (Canada, ministère des Finances 2007).

Au nom de cette recherche de « l'efficacité de l'aide »(MAÉCD 2009), ces politiques ont pour effet de créer une explosion du financement et du recours à l'expertise canadienne en développement international. En 2013, toujours dans un souci d'efficacité, et à la recherche de nouveaux partenaires financiers pour le développement, le gouvernement conservateur du Canada s'estime « résolu à renforcer la collaboration avec les acteurs du secteur privé, qu'ils soient canadiens ou étrangers, comme partenaires dans l'obtention de résultats de développement liés à ses priorités thématiques »(Canada 2013). Pour ce faire, le gouvernement conservateur procède à l'intégration de l'ACDI jusque-là responsable des programmes de développement international, à son ministère des Affaires étrangères et du commerce. Ainsi, l'aide canadienne au développement et les organismes qui y participent n'échappent pas à cette transformation du secteur de l'aide au développement. L'arrivée en masse des capitaux privés (CCCI 2001, OCDE 2015), la concurrence grandissante pour l'obtention des fonds publics d'aide au développement affecte particulièrement sa composition et la stagnation de la contribution publique canadienne à l'aide au développement (OCDE 2015), ce qui transforme la façon dont l'aide se traduit sur le terrain et ce, particulièrement en Afrique (Black 2009; Brown 2013), principal récipiendaire de l'aide publique au développement du Canada (Bhushan 2013). La plupart des experts canadiens en développement international œuvrent dans un contexte grandement influencé par les discours et les outils techniques de développement émanant des institutions de Bretton Woods (BM, FMI), de l'organisation de l'aide canadienne (ACDI, MAÉCI) et de la politique étrangère de l'État 
canadien en matière de développement qui est influencé par les politiques adoptées à l'OCDE.

En effet, plusieurs politiques, programmes et orientations de l'ACDI énoncés plus haut sont directement inspirés de travaux ou de recommandations de la Banque mondiale ou encore d'accords conclus dans le cadre de réunions de l'OCDE. En ce sens, l'expertise en développement international et les experts canadiens de diverses spécialisations venus régler les problèmes des «populations vulnérables » se trouvent à être un enjeu passé (Parpart 2003) et actuel important de l'aide canadienne au développement, soit une situation en constante évolution. Ainsi, s'intéresser aux experts canadiens dans le cadre de ce mémoire nous offre l'occasion d'examiner le contemporain de ces derniers, c'est-àdire la perception de leur présent, la perception de leur passé tel qu'ils se le remémorent aujourd'hui et celle de leur avenir tel qu'ils l'anticipent (Hilgers et Mangez 2014) dans un monde du développement en profonde crise et aux prises avec des changements radicaux de son mode de fonctionnement habituel (Essex 2012) sans pour autant laisser en plan sa relation avec le passé.

\section{I.2.e - Un enjeu pour les communautés d'Afrique subsaharienne}

Le Canada entretient depuis plusieurs années une importante relation d'aide au développement avec les pays d'Afrique subsaharienne. En effet, dans les dernières années, la proportion la plus importante $(38 \%)$ du budget de l'aide canadienne au développement est consacrée à l'Afrique subsaharienne (OCDE 2014). Or, si l'on en croit les récipiendaires de l'aide au développement autour du monde, la nature et la forme de l'expertise mobilisée pour le développement est un élément clé du déploiement de ce que certains qualifient de « système de distribution du développement » (Anderson, Brown et Isabella 2012) voir dans une perspective plus cynique et critique, d' " industrie du développement » (Hancock 1992; Van Rooy 1998; de Haan 2009). Les récipiendaires de l'aide au 
développement et les effets d'un projet ou d'un programme d'aide au développement sont souvent le centre d'intérêt de la recherche en développement. Des études sur les effets attendus et inattendus de l'aide sur le récipiendaire existent (Swidler et Watkins 2009; Watkins et Swidler 2013); elles permettent de dire que cette relation d'aide au développement continue et grandit. C'est particulièrement vrai dans le cas de l'Afrique subsaharienne qui offre l'occasion d'observer comment se déploie l'expertise canadienne en développement international en contexte africain. Peu de travaux se sont penchés sur la diversité et la nature des experts et expertises mobilisés dans ce contexte. Suite à notre recherche, nous croyons être en mesure de produire des informations qui peuvent être utiles pour les experts canadiens et les planificateurs de l'aide canadienne, mais qui peuvent aussi aider les récipiendaires à identifier les genres d'experts et d'expertise auxquels ils ont affaire. Enfin, de telles informations permettront aux partenaires africains sur place de déterminer leurs besoins en expertise et les formes de profits qu'ils peuvent tirer de la présence d'un expert canadien.

Si l'expertise en développement international est le lieu commun de polémique dans les grands cercles de discussion internationaux, tels les Sommets de la Terre ${ }^{5}$ ou encore les forums de haut niveau sur l'efficacité de l'aide ${ }^{6}$, et que cette question semble à tout le moins très étudiée sur le plan théorique, il importe de mettre en lumière autant le contexte de l'acquisition de l'expertise, que les conditions de pratique pour pouvoir mieux comprendre la mise en cause des institutions, des experts et autres acteurs du développement dans la relation entre l'expertise, le développement international et son échec. Bien qu'il soit un acteur de second ordre en matière d'aide publique au développement, le Canada présente les caractéristiques typiques d'un acteur occidental « sans passé colonial ». ${ }^{7}$ Mais, l'aide du Canada est

5 À tous les dix ans, les membres de l'ONU se rencontrent pour faire un bilan de environnementale de la planète. Historiquement on retiens le sommet initial de Stockohlm (1972), puis ceux de Nairobi (1982), Rio de Janeiro (1992), Johannesburg (2002) et de nouveau Rio de Janeiro (2012).

6 C'est notamment dans ces rencontres internationales que des ententes internationales tels que la Déclaration de Rome (2003), la Déclaration de Paris (2005), le programme d'action d'Accra (2008) et le Partenariat de Busan pour une coopération efficace au service du développement (2011) ont été conclue.

7 Nous aimerions souligner que le Canada se présente souvent sur la scène internationale comme une ancienne colonie 
assez typique de l'industrie du développement avec laquelle les organismes et les acteurs africains doivent composer. La littérature sur l'expertise en général nous enseigne que l'expertise en développement international doit être envisagée en prenant en compte son propre contexte. Ainsi, il devient pertinent de s'intéresser aux particularités du cheminement des experts canadiens, soit à l'impact du contexte sur l'acquisition des compétences et les conditions de pratiques des experts en développement sur le «terrain ». C'est à partir de ces enjeux que nous établirons nos questions de recherche.

\section{I.3 - Questions de recherche}

Dans ce mémoire, nous proposons pour bien comprendre l'expertise dans le contexte des institutions du développement international, de prendre du recul et de s'interroger sur la nature et le contexte du travail des experts. Pour ce faire, nous nous demanderons « comment devient-on expert?» Le processus qui mène à devenir expert est intéressant, car son étude permet d'identifier comment on en vient à être inclus dans le champ de l'expertise, comment on parvient à s'y maintenir, mais aussi comment on pourrait en être exclu.

Pour mettre en contexte ce « devenir expert », nous nous poserons des questions additionnelles. Comment est-ce que les experts pratiquent l'expertise en développement? Comment les experts interagissent-ils entre eux et avec les communautés visées par le développement? Comment s'établit la « légitimité » d'une expertise en développement international? Dans quelles situations fait-on appel à l'expert?

européenne émancipée qui n'a pas pratiqué le colonialisme. Nous sommes conscients qu'à l'image de plusieurs autres de ces pays «sans colonie » cette image est fausse et occulte plusieurs réalités notamment la réalité des peuples autochtones du Canada et son implication dans les conflits coloniaux pour le compte de la Grande-Bretagne. 
Nous sommes d'avis que ces questions nous aideront à mieux comprendre comment on devient expert en développement international de même qu'à mettre en lumière l'impact du parcours de l'expert sur la lecture du contexte lorsqu'il est appelé à utiliser son expertise pour faire des choix d'intervention et d'interprétation des situations rencontrées par les experts en développement international.

\section{I.4 - Objectifs de recherche}

Cette recherche a plusieurs objectifs. Tout d'abord, nous souhaitons mieux comprendre les processus de décisions qui poussent les agences nationales de développement international à faire appel à l'expertise. L'expertise semble encore avoir la faveur des organisations de développement, et ce, même s'il a été démontré que l'apport d'expertise peut entrainer du développement seulement dans certains cas, et encore sans qu'on puisse l'expliquer. Ainsi, on peut se demander si le recours à l'expertise n'est pas, en fait, un appel à l'autorité destiné à justifier les opérations de développement. En nous penchant spécifiquement sur la catégorie des « experts consultants », nous souhaitons comprendre comment ces acteurs imaginent leur rôle dans la représentation qu'ils se font de la réalisation, la réussite ou l'échec d'un projet de développement international et comment ils arrivent à cette conclusion. C'est à ce titre que nous nous demanderons comment ils se représentent le parcours qui les a menés à occuper des fonctions d'expertise.

Sur un plan plus général, cette recherche nous permettra d'identifier pourquoi certains schèmes de problématisation du développement ont tendance à revenir auprès d'une catégorie d'experts plutôt qu'auprès d'une autre. En effet, en développement comme dans d'autres domaines, l'expertise est essentiellement mobilisée par un expert pour identifier un problème de développement ou étoffer la 
problématisation d'une situation perçue comme problématique par d'autres acteurs et ce faisant, cadre les solutions envisagées. Or, ces choix sont faits à l'aide de critères bien spécifiques selon le type de connaissances mobilisées pour faire face aux situations problématiques. Ainsi, nous souhaitons comprendre dans quelles mesures les cadres d'analyse choisis consciemment ou non par les experts influencent leur capacité à envisager d'autres problématisations possibles. De cette façon, nous pourrons les aider à élargir leurs horizons analytiques à travers une meilleure compréhension des éléments qui limitent leurs analyses.

En dernier lieu, l'étude de l'expertise canadienne en développement international nous permet de rendre compte de la diversité des scènes affrontées par l'expertise canadienne en développement international en Afrique subsaharienne ainsi que la diversité des activités engagées et des stratégies adoptées par les acteurs pour y faire face en employant les différentes ressources à leur disposition. De cette façon, nous croyons être en mesure de donner la parole aux « experts consultants » pour qu'ils s'expriment à propos des défis rencontrés et de leur travail d'expert. En effet, nous sommes d'avis que les experts sont conscients des transformations de leur milieu et qu'ils sont en mesure d'identifier leurs forces et leurs limites pour collaborer à l'enrichissement de leurs outils analytiques.

Notre mémoire vise à contribuer à la fois aux sciences sociales et aux transformations de la société. Notre recherche nous permettra, nous l'espérons, de contribuer à l'exercice de critique et nécessaire du développement international pour ainsi parvenir à l'élaboration de politiques de développement plus démocratiques et de prises de décisions plus transparentes. Dans ce mémoire, nous souhaitons participer au développement des connaissances sur le rôle joué par l'expertise dans le développement international tel que perçu par les experts consultants canadiens dans ce domaine. Dans une perspective plus large, nous espérons ainsi contribuer modestement à l'édifice de la sociologie de l'expertise. 


\section{Chapitre II - CAdRe ThÉORIQUe - LA ThÉORIE DU CHAMP}

Comme nous l'avons vu dans le chapitre précédent, la problématique de l'expertise comporte plusieurs niveaux de relations politiques, économiques et sociales dans lesquelles s'imbriquent des relations interétatiques, des relations interinstitutionnelles, des relations professionnelles et des relations interpersonnelles. En sociologie, la théorie du champ de Pierre Bourdieu propose des outils destinés à identifier la structure et la nature des relations entre des individus ou des groupes d'individus selon leurs différentes activités sociales. En effet, sur le plan macrosociologique, la théorie du champ permet à la fois d'identifier les relations de pouvoir qui ont cours dans un champ d'activité particulier et ce qui lui donne son caractère distinctif. Sur le plan microsociologique, la notion de champ permet d'apprécier le parcours social d'un individu en vertu des règles et principes en vigueur, qu'elles soient explicites ou implicites. Grâce à cette double propriété, la théorie du champ rend possible le dépassement la dichotomie entre structure et agentivité. Elle s'inscrit dans une mésosociologie qui tient compte à la fois du contexte social et de l'expérience individuelle. Grâce à ses qualités, la théorie du sociologue français nous semble la mieux adaptée pour remplir nos objectifs de recherche et répondre à notre question de recherche.

Ce chapitre présente les principaux aspects de la théorie du champ de Bourdieu. Nous exposerons d'abord les différents éléments de la théorie de Bourdieu qui nous serviront à décrire et analyser le champ de l'expertise en développement international. En conclusion, nous présenterons une brève revue des critiques de la théorie du champ. 


\section{II.1 - Émergence d'une théorie du champ}

Au début de sa carrière de chercheur, Pierre Bourdieu s'intéresse principalement aux logiques de domination et à la reproduction des dominations dans le système éducatif français. Tout en rejetant l'existentialisme de Sartre et en élargissant les concepts marxistes de lutte des classes et des capitaux, Bourdieu développe ainsi une série d'outils conceptuels et théoriques pour appuyer ses thèses : logique de domination, capital culturel, violence symbolique (Bourdieu et Passeron 1964; Bourdieu et Passeron 1970) et habitus (Bourdieu 1979a; Bourdieu 1980a) qu'il peaufine et précise au fil de ses travaux. Sans pour autant faire l'objet d'un ouvrage générique comme les concepts précédents, le champ émerge dans la tentative de Bourdieu de généraliser les principes mis au jour dans ses travaux sur l'éducation. Sur les traces d'Émile Durkheim, Bourdieu mobilise des outils conceptuels et méthodologiques développés par la science physique pour décrire les interactions sociales et les perturbations sociales. Par analogie au concept de champ développé en physique quantique, mais surtout inspirer de son application en psychologie (Hilgers et Mangez 2014), Bourdieu développe l'idée de champs de forces distinctes propres à l'espace du social. À l'image d'un champ magnétique par exemple, les champs de forces de Bourdieu ont une influence sur les objets sociaux en fonction de leurs propriétés individuelles. L'appel au champ permet ainsi d'expliquer que la logique de domination suit les principes généraux évoqués précédemment, mais aboutit à des manifestations particulières et spécifiques comme en font foi son application dans une multitude d'études de cas qui traitent tantôt des artistes (Bourdieu et Delsaut 1975; Bourdieu 1991; Bourdieu 1992), des intellectuels (Bourdieu 1984a), des élites (Bourdieu 1979b), des bureaucrates (Bourdieu 1993a), du monde des affaires (Bourdieu 1997a), du patronat (Bourdieu et Martin 1978), du droit (Bourdieu 1986) et des politiciens (Bourdieu 1981; Bourdieu 1984b; Bourdieu 2011). 
Nous retiendrons des principaux ouvrages qui traitent du champ que ce concept permet d'identifier les structures particulières, les logiques de domination et les capitaux à l'enjeu d'un espace spécifique, dans lesquels interagissent quelques-uns des principaux autres concepts (habitus, violence symbolique, capital culturel, social, économique et symbolique) de la sociologie bourdieusienne. Le concept de champ permet ainsi à la fois d'appliquer les outils d'analyse des logiques de domination à l'ensemble de la société, mais aussi d'identifier l'enjeu des luttes d'une activité sociale spécifique. Ainsi, pour Bourdieu, les propriétés à la fois statique et dynamique du concept de champ permettent de réconcilier deux dimensions autrement opposées dans les sciences sociales, c'est-à-dire à la fois reconnaître les structures dans une perspective de relatif déterminisme social et l'agentivité des individus dans une perspective de liberté d'action, de capacités individuelles et d'autodétermination.

\section{II.2 - Le champ est un microcosme social}

Le champ est un concept central des théories de Bourdieu qu'il définit comme « un microcosme à l'intérieur d'un macrocosme social » (Bourdieu 2000) où évoluent des agents qui se disputent un capital spécifique (Bourdieu 2002). Le monde est ainsi divisé et subdivisé en une multitude de champs, qui ont une structure commune dans lesquels il y a des dominants et des dominés qui sont déterminés selon une logique particulière propre à chaque champ (Bourdieu 1992; Bourdieu et Wacquant 1992). Le microcosme constitue ici le « terrain » d'un espace de « jeu » qui possède des éléments « invariants »; soit le fait d'être délimité par des frontières et déterminé par une structure particulière qui a ses propres règles, ses enjeux, ses luttes et un sens du jeu particulier qui favorisent l'utilisation de stratégies propres à la position occupée dans le champ et aux formes de capitaux à l'enjeu (Bourdieu 2013).

Pour faire l'étude d'un champ, il faut procéder à un travail d'inventaire de l'utilisation de la notion 
à l'étude lors de laquelle on recherche les « invariants ». Ces champs jouissent d'une certaine autonomie et sont en mesure de se soustraire par moment aux intérêts extérieurs, cependant, ils ne sont pas immunisés et subissent à différents degrés l'influence des luttes économiques, politiques et sociales externes qui caractérisent l'espace social observé (Bourdieu 1993b). Lorsqu'on construit un champ particulier, la méthode bourdieusienne place comme priorité de définir le concept choisi en tenant compte de la notion de variation de définitions qui peuvent exister ainsi que des différents champs et structures dans lesquels on les retrouve (Bourdieu 2013). C'est par la rencontre d'agents qui évoluent dans le champ observé que l'on peut identifier la nature des « invariants ».

\section{II.2.a - Les frontières du champ}

Lorsqu'on délimite un champ, Bourdieu mobilise le concept de frontière dans le sens où les limites du champ sont mobiles dans le temps et parfois diffuses, car elles sont l'enjeu des luttes (Bourdieu 2013). Pour clarifier cette délimitation, il faut clarifier le « dedans » et le « dehors » du champ. De manière invariable, les frontières d'un champ ont une forme unique et mathématique, c'està-dire qu'elles ne sont pas binaires, mais plutôt qu'elles tendent vers l'infini. Tel un jeu de poupées russes dont les frontières sont diffuses, un champ s'inscrit d'abord dans un espace social particulier qu'il convient de délimiter. C'est à partir de cet élément qu'on trace les frontières géographiques du champ observé. 


\section{Espace social}

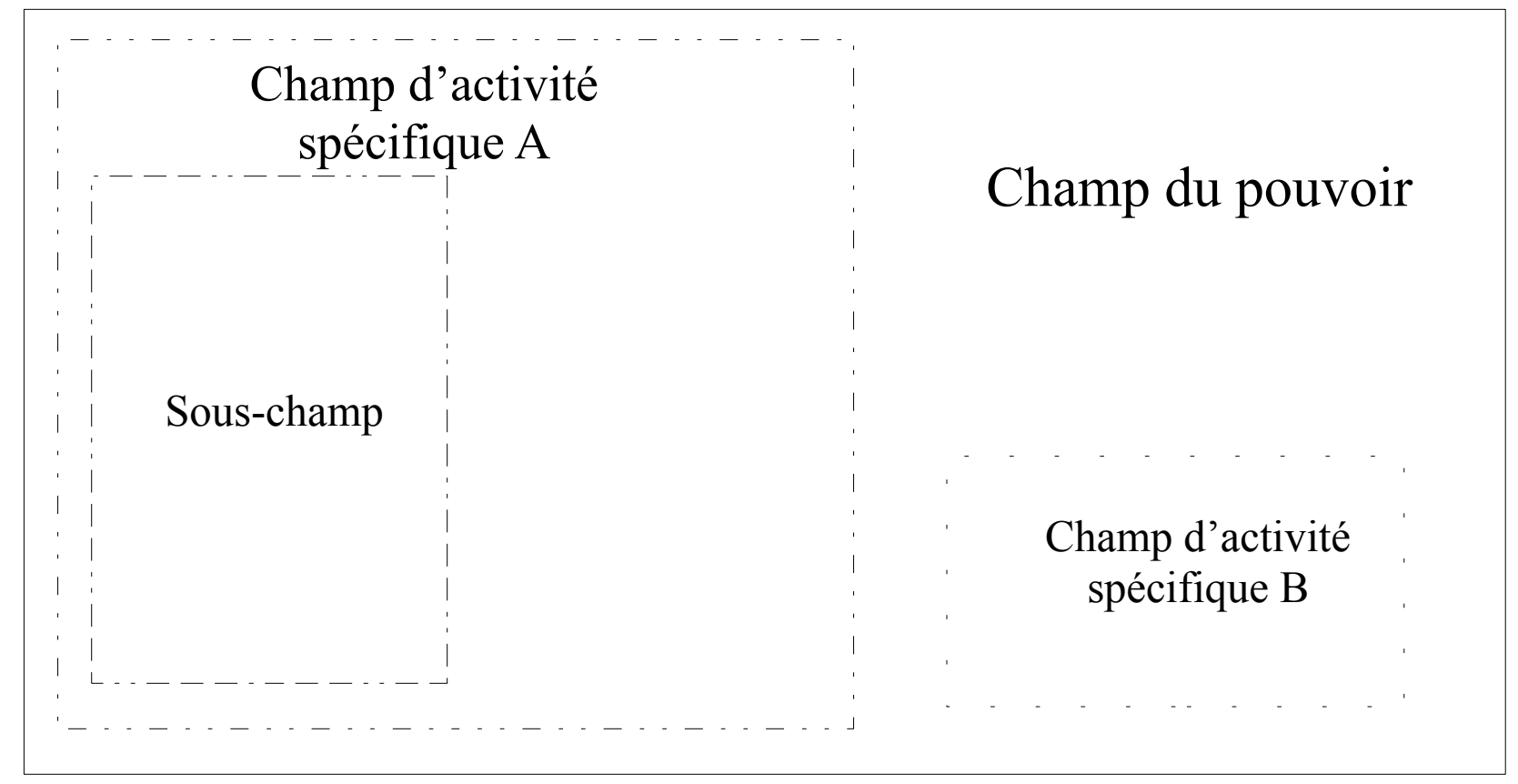

Légende

Frontière de l'espace social

Frontière du champ du pouvoir

Frontière du champ d'activité spécifique $\mathrm{A}$

Frontière du champ d'activité spécifique $B$

Frontière du sous-champ

\section{II.2.b - La structure du champ}

La structure d'un champ est un état du rapport de force, d'une lutte entre des agents et institutions engagés dans l'accumulation d'un capital spécifique. C'est le lieu d'une lutte qui sur le plan général a pour enjeu le monopole de la violence légitime et pour capacité celle de modeler la structure de distribution du capital spécifique au champ (Bourdieu 2002, 113-120). « Où [i]1 y a des lois générales

8 Librement inspiré de Bourdieu 1991 p.12 
des champs [...] dans tout champ on trouvera une lutte, dont il faut à chaque fois rechercher les formes spécifiques, entre le nouveau entrant qui essaie de faire sauter les verrous du droit d'entrée et le dominant qui essaie de défendre son monopole et d'exclure la concurrence » (Bourdieu 2002, 113). Ainsi, différentes combinaisons de capitaux économiques, culturels et symboliques sont utilisées ou convoitées en fonction de la structure du champ et de la lutte qui structure ce dernier. Ces capitaux composés de biens matériels, de relations sociales, de connaissances ou de sanctions officielles telles des diplômes sont ensuite transformés en différents capitaux de légitimité donnant accès au capital spécifique valorisé du champ et ultimement à du pouvoir. Invariablement, un champ a une forme d'homologie structurale avec les champs ou les espaces sociaux dont il fait partie. C'est-à-dire qu'un champ comporte des dominants et des dominés qui ne sont pas les mêmes d'un champ à l'autre, mais qui correspondent au champ plus large duquel il fait partie. Cela rend possible l'influence de l'extérieur, mais permet aussi à un champ de garder un certain degré d'autonomie. C'est-à-dire sa capacité à résister à certaines dominations. Plus cette autonomie est marquée, plus fréquente est possible l'observation du paradoxe suivant :

[...] l'existence de champs relativement autonomes, fonctionnant selon les mécanismes rigoureux et capables d'imposer aux agents leur nécessité, qui fait que les détenteurs de moyens de maîtriser ces mécanismes et de s'approprier les profits matériels ou symboliques produits par leur fonctionnement peuvent faire l'économie des stratégies orientées expressément et directement vers la domination des personnes. (Bourdieu 1980a, 226)

Ce sont ces règles, ces luttes et le degré d'autonomie qui donnent une structure au champ. Ainsi, l'étude d'un champ permet d'en analyser la structure interne, soit les structures de relation entre les groupes placés en situation de concurrence pour la légitimité (Bourdieu 1991). Ces luttes et ces règles produisent un registre d'agents qui usent de stratégies générales selon la position occupée dans le champ. En règle générale, ceux qui sont dans un état déterminé du rapport de force monopolisent le capital spécifique et sont inclinés à des stratégies de conservation, alors que ceux qui en sont dépourvus 
ont recours à des stratégies de subversion (Bourdieu 2002, 199). Ces stratégies se trouvent utilisées, mobilisées et modulées selon un principe inconscient d'action: l'habitus.

\section{II.2.c - Les règles du champ}

Le champ est structuré et uni par trois règles de base qui sont 1) le droit d'entrée, 2) la doxa et 3) l'illusio. L'habileté à naviguer dans l'esprit de ces règles est déterminée par un sens du jeu généré par l'habitus.

\section{Le droit d'entrée : valider son accès au champ}

Pour accéder à un champ, il y a un droit d'entrée fixe qui se « paie » sous forme de possession de capital spécifique reconnue par le champ et de l'acceptation de certains principes propre au champ. À la différence du sport, un agent n'entre pas nécessairement dans le « jeu » de façon consciente. En effet, ce sont les règles du champ qui sélectionnent les agents possédant les habitus nécessaires pour assurer, tel un prix d'entrée, le bon fonctionnement du champ (Bourdieu 1980a). Ces règles structurent à leur tour la production, la transformation et la consommation de capital spécifique à chaque champ.

\section{La doxa : l'acceptation de la règle}

L'entrée dans un champ suppose l'acceptation commune de tous les agents en lutte d'une valeur ou d'un régime de valeur auquel tous adhèrent, peu importe la quantité de capital possédé par chacun, sous peine d'expulsion. C'est un « ensemble de croyances fondamentales qui n'ont pas besoin de s'affirmer sous la forme d'un dogme explicite et conscient de lui-même » (Bourdieu 1997b). La doxa c'est l'acceptation tacite des hiérarchies du champ et de sa manière de répartir le capital entre les 
agents.

\section{L'illusio : croire dans le « jeu»"}

Formée de la rencontre de la doxa et de l'habitus, l'illusio permet de s'investir dans le « jeu ». Cette rencontre des règles de base et de l'habitus génère le comportement qui permet de produire un intérêt à « jouer» le jeu et à croire en ce dernier. L'illusio, est une croyance que le « jeu » d'un champ vaut la peine d'être joué. C'est le critère d'entrée minimal d'un champ, ce que Bourdieu qualifie de « foi pratique ». Il s'acquiert ou se transmet souvent par des rîtes de passage, des examens et s'accorde aux présupposés - la doxa - d'un champ et qui suppose un investissement en temps dans le champ et pour celui-ci (Bourdieu 1980a). Par-dessus tout, « Dire en public la vérité du champ et ses mécanismes [...] représente le sacrilège par excellence » (Bourdieu 1991, 48). La plupart des agents sont conscients de cette illusio, mais préfère prendre plaisir dans l'inconscience de produire le principe même qui produit l'illusio.

\section{L'habitus : le sens du jeu}

La notion d'habitus développée par Pierre Bourdieu emprunte aux philosophies d'Aristote, d'Edmund Husserl et de Thomas d'Aquin (Wacquant 2010) et aux sociologies d'Émile Durkheim, de Marcel Mauss et de Norbert Elias (Gonthier 2014) . En utilisant cette notion, Bourdieu a comme objectif de fonder « une théorie de l'action posant que les agents sociaux ne sont pas des êtres passifs manipulés par des forces externes, mais des créatures agiles et habiles qui construisent activement la réalité sociale par le biais de catégories de perception, d'appréciation et d'action » (Wacquant 2010, 115). Cette théorie pose les jalons d' 
[...] un conditionnement associé à une classe particulière de conditions d'existence produisent des habitus, systèmes de dispositions durables et transposables, structures structurées prédisposées à fonctionner comme structures structurantes, c'est-à-dire en tant que principes générateurs et organisateurs de pratiques et de représentations qui peuvent être objectivement adaptées à leur but sans supposer la visée consciente de fins et la maîtrise expresse des opérations nécessaires pour les atteindre, objectivement « réglées » et " régulières 》 sans être en rien le produit d'obéissance à des règles et, étant tout cela, collectivement orchestrées sans être le produit de l'action organisatrice d'un chef d'orchestre.(Bourdieu 1980a, 88-89)

Ainsi, « l'habitus est un ensemble de dispositions acquises [qui] pose que la maîtrise pratique fonctionne en deçà de la conscience et du discours [dont] les structures conatives et cognitives socialement constituées qui composent l'habitus sont malléables et transmissibles parce qu'elles résultent d'un travail pédagogique. [Cet] habitus indique que les ensembles de dispositions varient en fonction de la position et de la trajectoire sociale » (Wacquant 2010, 115) d'un individu. Il s'agit en clair du «lieu » où se construit la culture d'un groupe et l'identité des individus (Boltanski 2005). L'habitus se trouve ainsi à avoir une propriété double à la fois individuelle et collective, qui permet à un individu de s'intégrer dans un champ ou une communauté de pratique et de s'en approprier la culture dans un processus d'apprentissage. L'habitus est le produit de l'accès à différentes formes de capitaux et donne lui-même accès à différentes communautés et à du capital social.

\section{II.2.d - Le capital: enjeux ultimes du champ}

Bourdieu s'inspire de la notion de capital économique marxiste (Marx 1976) et élargit la notion à toutes les activités sociales. Il ajoute au capital économique de Marx trois types de capital supplémentaires : le capital social, culturel et symbolique.

\section{Le capital économique}

En premier lieu, la sociologie bourdieusienne reprend la définition de capital comme définie par 
les théoriciens marxistes, soit un capital composé de biens matériels; tels une maison ou une terre, ou des revenus; tels un salaire, une rente, etc. Au terme capital, on associe le qualificatif d'économique pour le distinguer des autres formes de capital.

\section{Le capital social}

En second lieu, Bourdieu développe le concept de capital social soit « l'ensemble des ressources actuelles ou potentielles qui sont liées à la possession d'un réseau durable de relations plus ou moins institutionnalisées » (Bourdieu 1980a) par un agent. Le volume de ce capital dépend de l'étendue du réseau de liaison que l'agent peut mobiliser et du volume de capital possédé en propre par chacun de ceux auxquels l'agent est lié (Bourdieu 1980a; Bourdieu 1980b). Bourdieu réfère alors à l'appartenance à un groupe dont les relations sont mobilisables pour multiplier son propre capital. Cela peut, par exemple, être un club social ou une association professionnelle, un groupe d'anciens diplômés, des gens fréquentant une même conférence chaque année.

\section{Le capital culturel}

Ensuite, Bourdieu élabore le concept de capital culturel qui lui possède une forme plus complexe et se présente sous trois états. Premièrement, le capital culturel se présente sous la forme de «[...] $\underline{l^{\prime} e ́ t a t}$ $\underline{\text { incorporé }}$ c'est-à-dire sous la forme de dispositions durables de l'organisme [...] » (Bourdieu 1979a, 3), le capital culturel est dans cet état acquis à partir d'apprentissages, qui permettent d'intégrer des dispositions dans l'habitus de celui qui y investit le temps nécessaire. Deuxièmement, il est présent sous la forme d'un « [...] état objectivé, sous la forme de biens culturels, tableaux, livres, dictionnaires, instruments, machines qui sont la trace des réalisations de théories ou critiques de ces théories, problématiques, etc. » autrement dit des objets qui ont un caractère culturel spécifique qui leur sont 
associé. Troisièmement, il est également présent sous la forme d'un « [...] état institutionnalisé, forme d'objectivation qu'il faut mettre à part parce que comme on le voit avec le titre scolaire, elle confère au capital culturel qu'elle est censée garantir des propriétés tout à fait originales » (Bourdieu 1979a, 3) par exemple un diplôme, un certificat ou toute autre reconnaissance institutionnelle sanctionnant la satisfaction des critères reconnus.

\section{Le capital symbolique}

La quatrième et dernière forme de capital possède quant à elle un statut particulier parce qu'elle n'existe que dans un champ donné ou en lien avec celui-ci. Soit,

n'importe quelle espèce de capital (économique, culturel, scolaire ou social) lorsqu'elle est perçue selon des catégories de perception, des principes de vision et de division, des systèmes de classement, des schèmes classificatoires, des schèmes cognitifs, qui sont, au moins pour une part, le produit de l'incorporation des structures objectives du champ considéré, c'est-à-dire de la structure de la distribution du capital dans le champ considéré. (Bourdieu 1994, 160-161)

Le capital symbolique regroupe ainsi toutes les formes de capital qui, lorsqu'associées aux critères et principes d'un champ particulier, se trouvent valorisées. Son détenteur se voit presque automatiquement conférer une reconnaissance tels la légitimité, l'autorité, ou encore le prestige.

Cette subdivision du capital en de multiples formes permet à la théorie bourdieusienne d'expliquer deux paradoxes : la multitude d'endroits où l'accumulation de capital économique n'est pas le principal enjeu de la lutte d'un champ et la multitude d'endroits où la possession de capital autre qu'économique permet l'accumulation de capital économique. 


\section{II.3 - Revue critique}

L'habitus est certainement l'un des concepts les plus controversés et les plus critiqués de l'œuvre de Bourdieu. Son caractère à la fois individuel et collectif (Boltanski 2005) est son aspect le plus critiqué.

Pour les tenants de la rationalité économique, l'habitus constitue une rationalité trop déterministe. Les choix stratégiques d'agents seraient attribuables à des automatismes. Les tenants de la rationalité délimitée (Crozier 1971; Crozier et Friedberg 1977; Simon 1991; Kahneman 2003), voir la rationalité pure (Elster 1979; Becker et Murphy 1988; Coleman 1988) préfèrent expliquer le choix stratégique en ayant recourt au concept d'acteur individuel qui agit en fonction de ses intérêts et objectifs dans une économie de marché avec à l'enjeu, essentiellement un capital économique.

À l'opposé, les auteurs expliquant le choix individuel par sa détermination collective préfèrent utiliser la notion de réseau où les relations nouées par les acteurs entre eux, voire avec des institutions, jouent un rôle prépondérant dans leur réussite et leur quête de légitimité (Collins et Evans 2002; Rip 2003; Akrich, Callon et Latour 2006; Collins et Evans 2008; Evans 2008).

Pour d'autres, ce sont davantage les définitions différenciées des capitaux à l'enjeu qui ne sont pas hiérarchisés adéquatement et qui viennent jeter un flou sur l'exploitation qui a cours et qui aliène les travailleurs. Ainsi, pour les partisans du matérialisme historique, le seul capital qui prime au niveau de l'analyse de l'exploitation, c'est le capital économique (Marx 1976; Engels et Marx 1974; Debord 2006; Lukács 1960). De cette façon, Bourdieu, élargit la notion de capital en fractionnant le concept global en catégories et fait de même avec le concept de classe. Cette complexification du cadre d'analyse se 
trouve alors à nier la réalité matérielle de production du travail d'expertise. En conséquence de quoi, la lutte de classes s'estompe au profit de la lutte de champ qui met en exergue la dénotation des contradictions internes et contextuelles du champ plutôt que de dénoncer la structure contradictoire de la société elle-même.

À l'opposé d'autres considèrent le capital culturel et symbolique comme la principale source de pouvoir dans une société et considèrent que le champ de Bourdieu ne lui accorde pas l'importance qu'il mérite. Ils avancent que la construction sociale de l'ethnicité et des cultures varie dans le temps en fonction des contextes historiques et occupe un rôle plus important que le capital économique ou social dans la domination (Gilroy 1991; Hall 1993; Bhabha 1994; Roediger 2002; Della Faille 2012). En effet, déjà au début de la décolonisation de l'espace géographique de l'Afrique, la construction de l'identité coloniale et postcoloniale de plusieurs « ex-colonies » incorpore une importante part de la représentation, de l'imaginaire et de l'accentuation des différences culturelles (Said 1979; Fanon 2002; Césaire 2004), ce qui met en lumière l'importance du capital symbolique. Ainsi pour ces auteurs et ceux des cultural studies, c'est en se réappropriant son identité propre, et en dénonçant le symbolisme du colonialisme qu'il est possible d'activer le capital social et de se réapproprier le capital économique nécessaire à l'émancipation des peuples opprimés.

\section{II.4 - Ce qu'on en retient : le Champ de l'expertise en développement international}

Si l'on s'inspire des travaux de Pierre Bourdieu sur le champ, le monde des institutions du développement peut être considéré comme un espace social structuré où des luttes ont cours. Ces luttes ont pour enjeux l'accumulation de capital sous différentes formes : culturel, économique, social, symbolique. Ce monde est composé de plusieurs champs, dans lesquels il y a des dominants et des 
dominés qui sont déterminés selon une logique particulière propre à chaque champ (Bourdieu 1992; Bourdieu et Wacquant 1992). On trouve parmi ces champs, celui de l'expertise en développement international. Les logiques internes des champs et ces rapports entre dominants et dominés se trouvent conditionnés par un régime acquis qui détermine l'action des agents œuvrant dans le champ : l'habitus. Ces champs jouissent d'une certaine autonomie et sont en mesure de se soustraire par moment aux intérêts extérieurs. Cependant, ils ne sont pas immunisés et subissent, à différents degrés, l'influence des luttes économiques, politiques et sociales externes, notamment l'influence de l'État, sa bureaucratie (Bourdieu 1993a) et du néolibéralisme (Collier 2012; Hilgers 2012).

\section{II.4.a - La théorie du champ pour notre recherche}

Dans la logique que nous venons de présenter, l'expertise en développement international forme un champ distinct possédant ses propres logiques de domination et dont les individus et les groupes le composant possèdent leur propre habitus particulier.

En conséquence, dans ce mémoire, en nous inspirant de la théorie du champ de Pierre Bourdieu, nous allons d'abord définir succinctement l'espace social dans lequel nous comptons ancrer un champ de l'expertise en développement international. Il conviendra ensuite d'en identifier les règles de base et leur usage lors de la construction du champ que nous souhaitons observer. En paraphrasant Bourdieu (Bourdieu 2013), nous définirons l'expertise en développement international en tenant compte de la notion de variation de définitions qui peuvent exister, des différents champs et des structures dans lesquelles on les retrouve. Pour ce faire, nous procéderons à un travail d'inventaire de l'utilisation de la notion à l'étude dans lequel on doit être à la recherche des constantes dans le champ de cette expertise en développement international. 


\section{Chapitre III - CAdRe MÉThOdologiQue}

Ce chapitre présente les différents aspects méthodologiques de notre recherche. Nous aborderons d'abord les justifications de notre choix méthodologique, une description des entretiens administrés et de leurs limites, une description de l'échantillon et de ses limites.

Nous avons arrêté notre choix méthodologique sur l'entretien de recherche semi-dirigé. Notre recrutement a été effectué par échantillonnage dit « boule de neige ». Le guide d'entretien utilisé a été affiné au fil des entretiens avec les experts consultants afin de refléter leur représentation du monde.

\section{III.1 - Justification du choix méthodologique}

En mobilisant un cadre théorique s'inscrivant dans la théorie du champ de Bourdieu et en raison de la flexibilité méthodologique du cadre théorique une foule de méthodes aurait pu être envisagée. Afin de mieux saisir quels sont les modes de fonctionnement de la mobilisation de l'expertise pour l'organisme contractant du point de vue des experts, l'analyse qualitative des discours produits par les experts consultants nous semblait la mieux adaptée pour préparer l'esquisse du champ de l'expertise en développement international sur les plans scientifiques et pratiques.

\section{III.2 - Avantages pratiques de la méthodologie adoptée}

D'un point de vue pratique, l'éventail de moyen et de temps à notre disposition étant limité, nous 
avons privilégié l'entretien de recherche. Cette méthodologie nous semblait pouvoir offrir le meilleur accès aux savoirs produits tout en considérant les investissements nécessaires en matériel et en temps de la part du chercheur (Blanchet 2005). L'entretien semi-dirigé nous a permis de tirer profit du réflexe de politesse des personnes rencontrées lors des entretiens, soit cet effort de courtoisie qu'enjoint la culture occidentale de répondre aux questions d'une personne intéressée à eux ou leur passion de leur désir d'influencer le monde ou encore de leur besoin de parler qui ont été assouvis par l'entretien (Grawitz 2001, 660). En tirant profit de ces caractéristiques, on peut ainsi aborder les sujets plus sensibles (Blanchet 2005) dans la communauté restreinte de ceux qui pratiquent le développement. Le choix de l'entretien semi-dirigé permet aussi une adaptabilité de la recherche au fil de sa conduite. Cette caractéristique nous a permis d'adapter notre questionnaire en fonction du déroulement des entretiens afin de tenir compte du discours de la personne rencontrée et de bien comprendre la perspective (Savoie-Zajc 2009) des consultants experts sur le champ des experts en développement international.

\section{III.2.a - Avantage scientifique de la méthodologie adoptée}

Du point de vue scientifique, nous avons souhaité mettre l'accent sur les expériences professionnelles et personnelles des consultants experts parce qu'eux seuls sont à même de nous éclairer sur leurs perceptions à propos de ce qu'ils estiment que l'ACDI, le MAÉCD et les décideurs font de leur travail. À cette fin, l'entretien semi-dirigé nous permet de profiter d'une foule d'avantages du point de vue de la qualité des informations produites : il fait ressortir un ou plusieurs discours chez les personnes rencontrées concernant les sujets que l'on souhaite aborder de même qu'il fait ressortir de l'information que l'on n'aurait pas envisagée grâce à la liberté qu'il permet (Grawitz 2001, 645), tout en permettant de traiter des sujets plus sensibles (Blanchet 2005). À cette fin, l'administration 
d'entretiens de recherche semi-dirigés pour stimuler les discours sur le développement nous permet de tirer profit de la richesse qualitative et du plus important détail des informations recueillies. Les qualités descriptives et la complexité des discours produits serviront à une co-construction des réalités des experts. L'entretien semi-dirigé, tout en identifiant les opinions, les conceptions de l'expertise, la perception du rôle de l'expert, les positions idéologiques et les stratégies utilisées par les experts (par exemple pour se faire valoir, faire passer des messages, contrer certains blocages, etc.) permet de saisir les discours afin d'élaborer une esquisse des réalités existantes.. Nous avons dressé un paysage du monde des professionnels du développement à partir de la comparaison de ces différentes constructions du réel. La méthode nous autorise ainsi à poser plusieurs questions en lien avec notre sujet qu'il serait difficile de traiter par d'autres que celle-ci. Effectivement, l'entretien procure un accès direct à l'expérience (Savoie-Zajc 2009, 356), des consultants experts. De plus, sur le plan éthique, l'entretien confère une certaine légitimité à l'enquêteur, car son « sens est négocié entre les interlocuteurs [pour tenter] de bien comprendre la perspective de l'autre grâce à une relation interpersonnelle établie » (Savoie-Zajc 2009, 356). Nous avons ainsi négocié la tenue et la teneur de chacun des entretiens évoqués dans ce mémoire.

\section{III.3 - L'entretien semi-dirigé}

L'entretien sollicité a pris la forme d'un « ... entretien entre deux personnes, un interviewer et un interviewé, conduit et enregistré par l'interviewé; ce dernier ayant pour objectif de favoriser la production d'un discours linéaire de l'interviewé sur un thème défini dans le cadre d'une recherche » (Blanchet 2005). Ainsi, nous avons rencontré ces personnes dans le cadre d'un entretien de recherche sur le thème de l'utilisation de l'expertise en développement international dans le but d'aider à produire les données nécessaires à une étude des représentations sociales des experts en cours actuellement dans 
le monde du développement. Cette sollicitation permet l'élaboration d'un « savoir socialement communicable et disputable » (Blanchet 2005, 84) que nous souhaitons interpréter. Ce dernier relate les réalités des pratiques expertes en développement international à travers la construction d'un discours sur l'expertise et le développement international.

\section{III.3.a - Description du guide d'entretien}

Les entretiens semi-dirigés, envisagés comme outil de collecte de données principales, ont été menés avec un guide d'entretien'. Le guide d'entretien a comme utilité de permettre au chercheur d'aborder les éléments qu'il souhaite examiner et de garder un certain contrôle sur l'entretien tout en donnant assez de liberté à la personne rencontrée pour aborder des sujets que le chercheur n'avait pas envisagés. Ci-dessous, nous décrivons les différentes sections de ce guide d'entretien. Aux fins de notre recherche, nous avons divisé le questionnaire en quatre sections que nous avons intitulées : «Quelques questions sur vous», «Parlons des experts», « Parlons de votre travail avec les agences de développement international» et «Parlons de l'aide au développement». Nous expliquons dans les paragraphes qui suivent la structure et le processus d'administration du guide.

La section «Quelques questions sur vous » du guide d'entretien a d'abord été mise en place dans le but de mettre les personnes rencontrées en confiance. Avant de débuter le questionnaire nous avons présenté la procédure à la personne recontrée,

« [assurer] l'anonymat de l'entretien, indiquer que l'enquêté a été déterminer par échantillonnage, éclairer l'enquêté sur ce que l'on attend de lui ; insister sur le fait qu'il ne s'agit pas d'un examen comportant de bonnes ou de mauvaises réponses, mais [que] tout ce que pense l'enquêté est intéressant [et le] rassurer sur la portée de ce qu'il va dire, sans [en] minimiser l'importance.» (Grawitz 2001, 661)

9 Voir Annexe 3 p.164 
Nous comptons mettre à l'aise la personne rencontrée à l'aide de question relative à son statut (Montane 2002). Notre recherche vise un type d'agent assez particulier qui forme une sorte d'élite sociale bien au fait des enjeux de la recherche en science sociale, mais ayant aussi l'habitude d'être sollicitée pour ses connaissances ou ses aptitudes spécialisées. En flattant modérément l'égo des personnes rencontrées lors des entretiens, on souhaite ainsi se prémunir contre la fuite polie et gagner leur confiance (Grawitz 2001, 659).

Une fois la personne rencontrée mise en confiance, nous passerons à la Section «Parlons des experts » du guide. À cette étape de l'entretien, nous souhaitons amener indirectement la personne à répondre à nos questionnements ce qui « implique [...] que le sens réel de la question ou de la réponse peut être différent de son sens apparent. Cette signification, supposée réelle, est obtenue à partir des renseignements fournis par l'enquêté, sans qu'il se doute de l'interprétation à laquelle ceux-ci doivent conduire » (Grawitz 2001, 643) parce que « [1’] on considère avant tout qu'il existe un écart entre ce que les gens disent et ce qu'ils sont ou font et c'est cette différence qui fait l'objet de la recherche. » (Grawitz 2001, 654) Graduellement, les questions seront précisées par l'enquêteur afin d'approfondir le sens des significations que la personne donne à ses réponses. De plus, nous allons sonder la personne rencontrée en la stimulant pour qu'elle exprime son opinion sur les acteurs généralement considérés comme des acteurs clés du milieu du développement international soient les ONG, les professionnels du développement, les agences nationales et internationales (Freyss 2004).

Par la suite, nous allons aborder la section «Parlons de votre travail avec les agences de développement international » du guide. À cette étape du questionnaire, nous souhaitons mieux comprendre comment l'expert conçoit son rôle dans le cadre des projets de développement effectués à l'étranger. À partir de cette étape, nous abordons plus précisément les différents aspects en lien direct 
avec le travail de la personne : ses interactions au travail, les choix qu'il fait, les extensions du travail dans la vie privée, etc.

Pour clore l'entretien nous passerons à la section «Parlons de l'aide au développement» du guide. À partir de ces questions, nous souhaitons sonder le consultant expert sur son avis concernant l'aide au développement et celui sur les critiques que l'on peut adresser à l'expertise.

\section{III.4 - Limites de la méthode}

Selon Blanchet, l'entretien de recherche possède trois faiblesses principales : l'absence de règles de conduite, son caractère interlocutoire et le statut scientifique indéfini des données produites auxquels nous ajouterons les limites que l'on peut imputer aux attributs du chercheur lui-même.

\section{III.4.a - Son caractère interlocutoire}

Le qualificatif de «non-dirigé » de l'entretien occulte le sens réel du dispositif de l'entretien, soit son caractère interlocutoire (Blanchet 2005 citant Bourdieu, Chamboredon et Passeron 1968; Pages 1970 Blanchet 1982). Le fait que les entretiens font, en quelque sorte, l'objet d'une préparation a un effet de cadrage sur les conclusions possibles de la recherche. Cet état de fait limite grandement les applications possibles de ladite recherche. En effet, la construction du questionnaire cadre en grande partie les sujets abordés et permet au chercheur de ne pas trop s'éloigner de ceux-ci. Cependant, cet effet de cadrage peut occulter des éléments des réalités que nous souhaitons observer et du monde que l'on souhaite révéler.

Le caractère interlocutoire de l'entretien est probablement l'une des appréhensions principales 
des personnes rencontrées. En effet, pour se protéger contre des conclusions qu'elles pourraient estimer négatives, elles peuvent, consciemment ou inconsciemment, se prémunir de mécanismes de défense naturels comme la fuite, la rationalisation, la projection, l'identification et le refoulement (Grawitz 2001, 659-660) Tous ces mécanismes de protection sont susceptibles d'entrainer l'analyse sur une mauvaise voie, voire de priver le chercheur d'informations pertinentes à son analyse. Les données recueillies ne reflètent donc qu'une partie du monde envisagé par le chercheur.

\section{III.4.b - L'absence de règles de conduite}

Les inconvénients posés par le caractère interlocutoire de l'entretien exigent de mobiliser un questionnaire semi-dirigé offrant une souplesse pour tirer profit de la fluidité de la discussion. Cette flexibilité permet de faire baisser « la garde » de la personne et ainsi son utilisation des mécanismes de défense naturelle. (Grawitz 2001, 659-660). Or, cette parade ne fait que changer la nature de la limite à surmonter. En optant pour un questionnaire ouvert caractérisé par l'absence de règles précises concernant la conduite de 1'entretien (Blanchet 2005, 85 citant Roethlisberger et Dickson 1943), il serait illusoire de croire que nous avons suivi scrupuleusement les mêmes règles d'administration des questionnaires étant donné l'ensemble de variables à contrôler (disponibilité des personnes, lieu de l'entretien, temps de préparation de l'personne à rencontrer, etc.). Bien que nous ayons tout mis en place afin d'administrer de la même façon le questionnaire aux personnes rencontrées en suivant un guide, le caractère différent des diverses expériences ont fait diverger de manière unique, le niveau de langage utilisé, la manière de poser les questions, leur ordre ou encore les questions

d'approfondissement. De plus, chaque entretien a apporté son lot de questions supplémentaires qui ont été ajoutées aux guides au fur et à mesure de l'enchainement des entretiens. Les conclusions tirées sont donc difficiles à systématiser, car chaque stimulus est isolé. 


\section{III.4.c - Le statut scientifique indéfini des données produites}

Dans un cadre d'entretien avec une telle fluidité, le statut scientifique des données produites reste indéfini (Blanchet 2005, 85 citant Friedman 1946). Certes, la subjectivité de l'entretien (Blanchet 2005) ne permett pas d'arriver à une conclusion définitive, par contre, il existe pour chacun des entretiens une différenciation entre l'information extraite par le chercheur et l'information donnée par la personnes rencontrées lors des entretiens (Blanchet 2005, 82). En effet, c'est dans la l'interprétation de la comparaison des expériences relatées dans les entretiens que réside la puissance analytique de l'entretien semi-dirigé. Elle permet ainsi de systématiser certains éléments expériences et d'identifier le contexte des vécus et les perceptions semblables décrits grâce aux détails et au caractère unique des expériences.

\section{III.4.d - Les capacités du chercheur}

Ainsi, si l'absence de règle permet de faire face aux réticences de la personne rencontrée et l'acceptation du caractère indéfini permet de tenir compte de la diversité des expériences, dans certains milieux étudiés les sujets peuvent avoir une réticence à adopter une position idéaliste ou cynique (Becker 1956), réduisant le spectre des discours produits. Conséquemment, il peut être difficile de faire ressortir celle-ci de leur pensée. Bien que la flexibilité de l'entretien semi-dirigé offre plus d'occasions de déjouer ces mécanismes de défense, ultimement, la capacité de parer ces derniers se limite dans les compétences d'analyse du chercheur. L'utilisation d'un ou de plusieurs mécanismes peut rendre difficile, voire impossible, l'analyse des données recueillies (Grawitz 2001, 659-660). L'entretien semidirigé permet plus de mises en situation propices à la levée des défenses et à la production d'informations pertinentes d'un point de vue scientifique. Cependant, en calibrant l'outil de collecte pour en optimiser la flexibilité, on limite grandement la comparabilité des données ainsi que la 
possibilité de généraliser les conclusions issues de ce corpus.

En dernier lieu, les compétences d'un débutant en matière de conduite d'entretiens peuvent produire leurs lots d'imprécision. Ces entretiens ont tous pris place dans un espace-temps précis et unique où la maitrise du questionnaire par le chercheur peut s'avérer différente d'un cas à l'autre. En ce sens ceci constitue une limite à la systématisation de la recherche. Conséquemment, en raison de la petite taille de l'échantillon et du nombre d'entretiens mené avec des sujets réels du milieu, il existe une variance importante dans la maitrise qualitative du guide d'entretien et dans la qualité des entretiens.

\section{III.5 - Processus d'échantillonnage}

Dans le but de limiter l'impact de ces faiblesses dans la méthode choisie et de tirer profit de ses forces, nous avons adopté un processus d'échantillonnage rigoureux. Dans la planification de cette recherche, nous avons établi une liste des critères théoriques et logistiques destinés à identifier les personnes à approcher, leur lien avec les buts de la recherche (Savoie-Zajc 2007). Nous exposerons ensuite les limites du processus et les considérations éthiques à prendre en compte.

\section{III.5.a - Critère de sélection de l'échantillon}

Sur le plan des critères de sélection, la théorie du champ impose de ne pas présenter une définition ou une description préalablement construite de notre objet de recherche et nous enjoint plutôt à «s'en tenir à une description utilisant le vocabulaire indigène qui restitue les catégories de pensée indigène » (Bourdieu 2013). En utilisant les termes et expressions du milieu, on permet l'émergence d'une variété des définitions propres à la diversité d'activités, d'orientation et de disciplines qui se 
réclament du développement international. Nous nous sommes donc inspirés des termes et exigences présents dans les offres d'emplois ou de volontariat mis en ligne par des organismes et des agences à la recherche d' « experts », d' « évaluateurs », de « consultants » ou de « coopérants » en développement international pour les associer à l'espace géographique ciblé dans le cadre de cette recherche. Par ailleurs, nous avons pris soins de retenir des personnes aux profils disciplinaires et domaines d'activités variés. Dès lors, nous avons seulement retenu les personnes qui répondaient à de tels critères généraux, soit les personnes qui :

- ont déjà travaillé avec des consultants ou agi comme consultant en développement international au cours de leur carrière.

- ont 5 ans ou plus d'expérience avec une organisation, une agence ou une entreprise canadienne œuvrant en développement dont la dernière année d'activité n'est pas plus lointaine que 2010 .

- ont déjà travaillé pour le compte d'un organisme canadien financé ou non par l'ACDI en Afrique de l'Ouest.

- sont titulaires d'un diplôme d'études de cycles supérieurs.

Après avoir satisfait à ces critères de sélection théorique, les personnes à rencontrer ont été choisies selon les critères logistiques suivants. D'abord, les rencontres en personne ont été privilégiées, lorsque le déplacement s'avérait impossible, la rencontre s'est faite par le biais du logiciel de communication Skype. Deuxièmement, nous avons contacté personnellement, par courriel ou par téléphone et tenu un entretien avec toutes les personnes qui ont accepté de nous rencontrer entre mai 2013 et décembre 2013. À la suite de quoi, nous avons tenu compte des disponibilités des personnes et des autres activités du chercheur dans le calendrier de recherche prévu. Les échéances du mémoire, la 
disponibilité des personnes, les coûts de la tenue d'un entretien (café, déplacement, etc.) ont ainsi eu un effet sur l'ordre rencontres. À la suite des entretiens, des personnes correspondant aux critères de sélection et référées par les personnes avec qui nous avons conduit un entretien de recherche ont été contactées. Ils ont été ajoutés à l'échantillon après l'obtention de leur consentement.

\section{III.5.b - Limite du processus d'échantillonnage}

Le déroulement d'une recherche peut être planifié, mais des éléments restent imprévisibles : «Planifier un échantillon, comme la recherche d'ailleurs, se fait en théorie. La recherche, elle, est toutefois vécue dans une réalité qui appose ses propres contraintes, souvent non prévues au départ » (Savoie-Zajc 2007, 109). Conséquemment, nous estimons que plusieurs facteurs ont influencé le processus d'échantillonnage lui-même. Ainsi, notre statut de chercheur étudiant a pu influencer négativement notre accessibilité aux personnes œuvrant dans les entreprises et les agences de développement. Nous avons en effet vu plusieurs de nos demandes poliment ignorées, voire simplement refusées. La plupart des personnes ayant refusé un entretien, ont invoqué devoir obtenir l'accord de leur employeur, un accord qui n'est jamais venu. Pour d'autres, faire cette simple demande consistait en elle-même un risque de préjudice. En effet, au moment de la cueillette de données, la censure des scientifiques (ICI.Radio-Canada.ca 2012), les suppressions de postes récurrentes depuis 2010 (Gaboury 2015) et selon notre perception, l'absorption de l'ACDI par le MAÉCD a suscité des préoccupations importantes chez les fonctionnaires qui pouvaient craindre de perdre leur poste dans ce cadre. Ces refus consécutifs nous ont poussés à changer de stratégie. Nous avons alors investi plus de temps dans la sollicitation des personnes souhaitant influencer les étudiants en développement international. Pour ces raisons, consultants indépendants, retraités, étudiants et consultants débutants en construction de leur réseau professionnel ont été ciblés. La stratégie adoptée face à cet obstacle a 
affecté la forme de l'échantillon et incidemment la nature des discours produits.

\section{III.6 - Précautions éthiques}

La recherche qualitative en science sociale pose de nombreux enjeux éthiques. En planifiant notre échantillonnage, nous avons rencontré trois préoccupations éthiques nécessitant des considérations par rapport aux effets indésirables que la recherche pourrait avoir sur les personnes participantes. Dans la première section, nous aborderons les préoccupations rencontrées et dans la deuxième, les précautions prises pour assurer l'éthique de la recherche.

\section{III.6.a - Préoccupations éthiques}

En premier lieu, la recherche en sciences sociales est indissociable de son sujet, c'est-à-dire que «La recherche sociale ne se pratique pas en vase clos. [...] Pour les populations étudiées (...), les risques de stigmatisation peuvent au contraire être assez lourds. » (Malouin 2002) Par exemple, il existe une possibilité que certains propos énoncés soient suffisamment distinctifs pour être attribués à des participants en particulier, ces derniers pourraient faire l'objet d'une stigmatisation et perdre leur emploi.

En second lieu, la transparence du processus de recherche doit être assurée. En effet, plusieurs aspects de celle-ci sont faits en l'absence des personnes rencontrées ou sans que les participants prennent part directement au processus. Afin d'assurer une transparence, il est par exemple impératif de prendre des mesures pour expliquer à l'ensemble des participants la manière dont les données sont traitées. 
En dernier lieu, les participants doivent comprendre en quoi ils font l'objet d'une recherche et consentir de manière libre et éclairée à sa conduite., Les participants peuvent par exemple avoir des questionnements par rapport à la pertinence d'une recherche sur l'expertise en développement. Avant de donner leur accord pour un entretien, nous nous devons de préciser la nature de la recherche, d'offrir sans pression aucune la possibilité de participer à celle-ci, de préciser et de prendre des mesures pour qu'ils soient maîtres de leur participation à la recherche.

\section{III.6.b - Précautions éthiques}

Considérant ces risques importants, nous avons adopté une série de règles et processus afin d'assurer la protection des participants. Tout d'abord, dans le but de protéger leur vie privée, nous avons choisi d'anonymiser les personnes rencontrées en leur attribuant un pseudonyme auquel nous faisons référence dans tous les documents de cette recherche. Toutes les données recueillies ont été gardées sur un disque dur protégé par un mot de passe et situé dans un local fermé à clé accessible uniquement par le chercheur.

Afin d'assurer transparence, consentement libre et éclairé et protection de la vie privée, chaque entretien s'est déroulé en suivant scrupuleusement le processus suivant : lors du premier contact, le projet de recherche a été brièvement expliqué avant de solliciter un rendez-vous. Nous avons laissé le choix du lieu et du moment de l'entretien à la discrétion du participant. Lors de ce rendez-vous et avant de commencer l'entretien, le chercheur a présenté plus en détail la recherche et ses objectifs aux personnes rencontrées. Le chercheur s'est assuré que celles-ci saisissaient bien l'objet de la recherche, ses objectifs et sa méthodologie. Pour ce faire, il a expliqué de manière précise et concise ses travaux de recherche et répondu à toutes les interrogations signifiées par la personne rencontrée. Il a ensuite 
précisé aux participants les dispositions dont ils pouvaient se prémunir à tout moment, soit : retirer leur contribution en partie ou en totalité et avoir accès à tout instant aux données les concernant. Par ailleurs, nous avons informé les participants des précautions prises par le chercheur pour protéger la confidentialité des données. En dernier lieu, nous avons offert au participant la possibilité d'autoriser l'utilisation des données recueillies dans une recherche connexe par le chercheur ou le directeur de cette recherche. Pour ce faire, le participant était invité à remplir un formulaire de consentement stipulant les mesures énumérées ci-dessus ${ }^{10}$. Au début de l'entretien, le chercheur a demandé le consentement verbal à l'enregistrement de l'entretien. À la fin de celui-ci, le chercheur a demandé au participant s'il connaissait d'autres personnes susceptibles de participer à la recherche, tout en obtenant son consentement verbal pour citer son identité lors des sollicitations. En dernier lieu, le chercheur a transmis ses coordonnées au participant et l'a informé qu'il aurait accès sur demande au rapport final de recherche.

\section{III.7 - Description de l'échantillon}

Notre échantillon est constitué d'un groupe de cinq hommes et deux femmes. Dans cette section, nous proposons d'abord une présentation de chaque participant et participante dans le but de fournir « le plus d'information contextuelle concernant entre autres, les caractéristiques des participants à la recherche et celle de leurs environnements de vie, de travail spécifique » (Savoie-Zajc 2009).

\section{Maurice}

Recruté par sollicitation, Maurice est un homme dans la mi-cinquantaine possédant près de vingt ans d'expérience à titre de consultant en développement international pour le compte de diverses

10 Voir Annexe 4 p.166 
agences gouvernementales nationales et internationales. Il est titulaire d'un diplôme d'études supérieures en anthropologie, d'une formation dans une école de développement international canadienne et est originaire de ce pays. Il a évolué en tant que consultant indépendant de projet de recherche et de projets de développement, se spécialisant au fil des années dans l'évaluation et le suivi de projets de développement. Pour ces raisons, il a été actif dans plusieurs types de projets et a travaillé comme consultant pour plusieurs types d'organisations. Il a œuvré principalement en Amérique du Sud, dans les Caraïbes et en Afrique de l'Ouest. Au moment de l'entretien, il se préparait à partir pour l'Afrique de l'Ouest afin de remplir un contrat d'évaluation de projet.

\section{Frank}

Recruté sur recommandation de l'entourage du chercheur et approchant la fin de la trentaine, Frank est un fonctionnaire public et forme un couple avec une femme travaillant pour le compte d'une agence gouvernementale de développement. Il a fait quelques contrats à titre de consultant et occupé des postes d'experts des questions économiques pour le compte de l'État canadien dans le cadre de forum et de ronde de négociation internationale touchant de près ou de loin le développement. Titulaire d'un diplôme d'études supérieures en économie de l'environnement, il se spécialise dans les questions environnementales. Il possède une petite compagnie de consultation en développement qu'il garde en réserve au cas où sa femme serait appelée à occuper un poste à l'étranger. C'est pour lui un « travail de fin de semaine » quand les occasions se présentent et c'est actuellement en « dormance ».

\section{Marie}

Recrutée par sollicitation, actuellement étudiante aux cycles supérieurs en sciences sociales et dans la soixantaine, Marie a plus de 20 ans d'expérience à titre de consultante et de coopérante en 
développement international. Ayant œuvré en Afrique et en Amérique latine et originaire de cette région, elle a aussi passé une bonne partie de sa carrière professionnelle à travailler au sein d'une agence gouvernementale de développement canadienne après avoir travaillé comme consultante. D'origine latino-américaine, elle est spécialisée dans les questions de genre et est maintenant accessoirement «à la retraite». Elle se concentre maintenant sur ses études et son sujet de prédilection : les problématiques de genre en développement international.

\section{Carl}

Recruté sur recommandation de l'entourage du chercheur, Carl a plus de vingt ans d'expérience en développement international et en coopération. Il a travaillé une quinzaine d'années à titre de coopérant sur les conseils exécutifs ou d'administration dans plusieurs grandes ONG. Lorsqu'il ne siégeait pas sur des conseils, il a voyagé en Afrique, en Amérique du Sud, en Asie du Sud-est et en Europe, où il a fait plusieurs contrats de consultation de courte et de moyenne durée. Titulaire d'un diplôme en pédagogie, il se spécialise dans les questions de gouvernance organisationnelle. Originaire du Canada anglophone et au début de la septantaine, il vient de prendre sa retraite, mais continue à fréquenter conventions, congrès, forums et symposiums sur le développement.

\section{Gérald}

Recruté par sollicitation, approchant la fin de la vingtaine, Gérald est actuellement étudiant aux cycles supérieurs en sciences sociales dans une école de développement international canadienne. Il possède une formation en sciences sociales et huit ans d'expérience en coopération internationale. Il a travaillé en Afrique de l'Ouest pour différentes ONG dans des stages de formation à la coopération et des stages de recherches avec des chercheurs africains en développement. Originaire du Canada, il se 
spécialise dans les questions de genre et de relations interculturelles et s'implique beaucoup auprès des ONG d'ici. Au moment de l'entretien, il préparait un terrain de recherche en Afrique de l'Ouest.

\section{Leonard}

Recruté sur recommandation d'un participant et approchant la septantaine, Leonard est anglophone et originaire du Canada. Il possède un diplôme universitaire en sciences politiques. Il a travaillé principalement comme consultant pour des agences gouvernementales et pendant quatre ans pour une firme privée de consultant. Spécialisé en économie politique, il a passé la majeure partie de sa carrière à l'étranger à effectuer des contrats à court terme. Désormais à la retraite, il s'implique dans un parti politique pour partager son expérience acquise dans le développement à l'étranger.

\section{Audrey}

Recrutée par sollicitation, Audrey approche la fin de la vingtaine et cumule près de neuf ans d'expérience en coopération internationale. Elle se spécialise dans la communication communautaire et l'intervention communautaire. Titulaire d'un baccalauréat en communication et d'une scolarité de deuxième cycle en droit international et œuvrant principalement dans le milieu communautaire au Québec, elle consacre chaque année une part importante de son temps comme volontaire consultante principalement dans le cadre de projet de plusieurs mois pour le compte d'ONG en Afrique de l'Ouest. Francophone et originaire du Québec, elle réside «quelque part entre Montréal et l'Afrique de l'Ouest. » 
Tableau B - Sommaire descriptif de l'échantillon

\begin{tabular}{|c|c|c|c|c|c|c|}
\hline Nom & Âge & Sexe & $\begin{array}{l}\text { Domaine(s) } \\
\text { d'activité }\end{array}$ & $\begin{array}{l}\text { Discipline de } \\
\text { spécialisation }\end{array}$ & $\begin{array}{l}\text { Région(s) de } \\
\text { spécialisation }\end{array}$ & $\begin{array}{l}\text { Activité } \\
\text { principale } \\
\text { actuelle }\end{array}$ \\
\hline Maurice & Mi-cinquantaine & $\mathrm{H}$ & $\begin{array}{l}\text { Développement } \\
\text { international }\end{array}$ & Anthropologie & $\begin{array}{l}\text { Caraibe et } \\
\text { Afrique de } \\
\text { l'Ouest }\end{array}$ & $\begin{array}{l}\text { Consultant } \\
\text { pigiste à son } \\
\text { compte }\end{array}$ \\
\hline Frank & Fin trentaine & $\mathrm{H}$ & $\begin{array}{l}\text { Fonction } \\
\text { publique et } \\
\text { discussions } \\
\text { internationales }\end{array}$ & $\begin{array}{l}\text { Économie et } \\
\text { environnement }\end{array}$ & «Le monde » & $\begin{array}{l}\text { Consultant } \\
\text { pigiste à son } \\
\text { c o m p t e e t } \\
\text { fonctionnaire }\end{array}$ \\
\hline Marie & Soixantaine & $\mathrm{F}$ & $\begin{array}{l}\text { Coopération et } \\
\text { développement } \\
\text { international }\end{array}$ & $\begin{array}{l}\text { Genre, droit et } \\
\text { développement }\end{array}$ & $\begin{array}{l}\text { Amérique } \\
\text { Latine et } \\
\text { Afrique }\end{array}$ & $\begin{array}{l}\text { Étudiante et } \\
\text { coopérante }\end{array}$ \\
\hline Carl & Fin soixantaine & $\mathrm{H}$ & $\begin{array}{l}\text { Développement } \\
\text { et coopération }\end{array}$ & $\begin{array}{l}\text { Gestion } \\
\text { d'organisation }\end{array}$ & «Le monde » & Retraité \\
\hline Gérald & Fin vingtaine & $\mathrm{H}$ & $\begin{array}{l}\text { Coopération } \\
\text { internationale }\end{array}$ & $\begin{array}{l}\text { Sciences } \\
\text { politiques et } \\
\text { genre }\end{array}$ & $\begin{array}{l}\text { Afrique de } \\
\text { l'Ouest }\end{array}$ & $\begin{array}{l}\text { Étudiant et } \\
\text { coopérant }\end{array}$ \\
\hline Leonard & Septantaine & $\mathrm{H}$ & Développement & $\begin{array}{l}\text { Économie } \\
\text { politique }\end{array}$ & « Le monde » & $\begin{array}{l}\text { Retraité et } \\
\text { militant dans } \\
\text { un parti } \\
\text { politique }\end{array}$ \\
\hline Audrey & Fin vingtaine & $\mathrm{F}$ & $\begin{array}{l}\text { Coopération } \\
\text { internationale }\end{array}$ & $\begin{array}{l}\text { Communication } \\
\text { sociale et droit }\end{array}$ & $\begin{array}{l}\text { Afrique de } \\
\text { l'Ouest }\end{array}$ & Coopérante \\
\hline
\end{tabular}

\section{III.7.a - Limites de l'échantillon}

L'échantillon mobilisé pour cette recherche possède de nombreuses limites. Entre autres limites, il est constitué de manière prédominante de personnes de sexe masculin (5 répondants sur 7) et âgées de plus de 50 ans. La faible représentativité des individus dans la trentaine et la quarantaine, et l'importante proportion d'individus de plus de 50 ans (4 répondants sur 7) limitent les applications de notre recherche. Par ailleurs, l'absence d'employés actifs dans une firme privée de consultation et le type d'échantillonnage choisi présente de nombreuses limites quant à la portée de notre recherche. La 
majeure partie des éléments vécus et racontés par ces acteurs du développement peut, dans la plupart des cas, remonter à des époques différentes du moment auquel les questionnaires ont été administrés et constituer un corpus propre à un traitement plus historique que sociologique en raison de l'âge de la majorité des participants. En effet, comme indiqué précédemment certains participants sont en fin de carrière ou ont récemment pris leur retraite.

La prédominance des hommes peut diminuer significativement la résonance des expériences et des réalités des femmes en raison de leur sous-représentation dans l'échantillon. L'absence d'employés de firme de consultation actuelle et l'expérience limitée des seuls répondants qui ont affirmé avoir une expérience dans ce milieu constituent l'un des points aveugles de notre recherche. Pour cette raison, il nous sera impossible de dresser un portrait équilibré du point de vue des entreprises œuvrant dans ce secteur. La méthode d'échantillonnage par « boule de neige » favorise la surreprésentation de certains éléments de réalités principalement en raison du fait que les recommandations de connaissance sont susceptibles de partager des caractéristiques sociales et des représentations semblables. Enfin, le caractère non probabiliste de l'échantillon et sa grandeur insuffisante écarte toute possibilité de généralisation des conclusions tirées de cette recherche.

Pour cette raison, les conclusions tirées de l'analyse du corpus produit à partir de l'échantillon présenté se limitent aux discours produits par les experts du développement, de la coopération et de la solidarité représentant le Canada ou des organisations gouvernementales ou non gouvernementales basées au Canada. 


\section{Chapitre IV - PréSENTATION Des RÉSUltats de L'enQuête}

Ce chapitre présente les propos des experts en développement international que nous avons rencontrés entre juin et décembre 2013. Nous organisons ici les propos recueillis à travers les entretiens sous la forme de grandes thématiques. Ces thématiques permettent d'explorer les différentes dimensions de la réalité des experts rencontrés afin de les replacer dans leurs contextes. Ce chapitre présente donc les propos des différents participants et participantes ${ }^{11}$ à notre recherche au sujet de 1) les impératifs de l'expertise en développement international, 2) les conditions dans lesquelles elle est pratiquée et 3) les choix que les experts sont amenés à faire face aux situations rencontrées dans le monde du développement.

\section{IV.1 - Les impératifs de l'expertise en développement international}

Selon les personnes interrogées pour notre recherche, en développement international, c'est le comportement attendu de l'expert, tel produire une analyse économique dans un rapport, qui en fait un agent légitime du champ pouvant y intervenir. En effet, au-delà de ce simple exemple, pour revendiquer cette légitimité, il faut avoir une série de comportements et de compétences minimales qui permet aux membres du champ de se reconnaître entre eux, mais aussi de se différencier de quelqu'un qui provient d'un autre champ. Selon les personnes rencontrées, ces habitudes et outils essentiels à son travail sont acquis notamment; tout d'abord à travers un parcours académique, d'une expérience des projets de

11 Certaines interventions rapportées ici ont été traduites de l'anglais (Carl et Leonard), car les entretiens ont été réalisés en partie en anglais afin de laisser la chance à la personne rencontrée de s'exprimer dans la langue qui lui convenait le mieux. 
développement et d'une socialisation aux codes du développement international. Par la suite, ces habiletés sont maintenues par la reconnaissance des compétences, par la démonstration des activités d'expertise et par un souci de constante valorisation du développement.

\section{IV.1.a - $\underline{\text { Avoir un parcours académique }}$}

En premier lieu, pour toutes les personnes rencontrées, il existe ce que l'on appelle un expert en développement international «véritable » ou « authentique ». Pigiste auprès de l'ACDI puis associé maintenant retraité d'une firme d'experts consultants en développement international, Leonard, spécialisé en économie politique, « ...comprend l'expert comme étant celui qui a des connaissances de pointe dans un domaine donné et qui en comprend les applications pratiques», alors que pour Marie, retraitée, ancienne fonctionnaire de l'ACDI et coopérante, « c'est un sujet qui a acquis des compétences par les études et par la pratique, et pour moi ce complément est important... ». Cependant, à l'instar de plusieurs autres (Audrey, Gérald, Leonard) Marie s'empresse de préciser qu'elle n'est pas toujours à l'aise avec le terme expert et va même jusqu'à proposer l'utilisation d'un autre terme (spécialiste) afin de répondre au critère d'authenticité :

Peut-être que je me trompe, par contre spécialiste, oui. J'ai travaillé sur le terrain, j'ai fait des études. Peut-être que si on fait la traduction de l'anglais expertise vient de là : OK, j'ai une expertise, un savoir-faire. C'est surtout quand je le lis avec le terme anglais que j'en ai une, mais je préfère quand on parle de spécialiste.

Une dimension essentielle d'un comportement valorisé dans le champ de l'expertise est donc la capacité de mobiliser des connaissances acquises dans le cadre de la poursuite d'études universitaires, idéalement, à travers une démonstration de la maitrise des méthodes d'analyse académique telles que l'expose Marie : 
Un spécialiste c'est, quelqu'un qui connait la problématique de façon approfondie. Il y a d'autres personnes qui ont une connaissance moins approfondie, donc elles ne sont pas spécialistes. Peutêtre que c'est des personnes qui ont un vécu très important, ont une réflexion qu'elles peuvent partager qui peut vraiment faire avancer des causes, mais qui ne sont pas capables de faire la même analyse qu'une personne qui a passé à travers des études universitaires.

Selon Leonard, être en mesure d'affronter et de réussir, par exemple, « l'exercice de révision par les pairs dans la publication d'articles et d'ouvrages permet, lui aussi, d'acquérir des outils d'évaluation et de les améliorer. » On évaluera ainsi la qualité du parcours académique à travers les diplômes obtenus, le nombre et la qualité des publications, conférences et autres communications que l'expert aura publiées.

Avoir un parcours académique constitue ainsi une épreuve dont la réussite permet d'identifier ceux qui ont le degré de curiosité, les outils analytiques appropriés et les expériences académiques nécessaires à l'application de l'expertise en développement international à des situations concrètes. Par contre, le critère du parcours académique ne suffit pas à atteindre le statut d'expert en développement international aux yeux des pairs. Pour ce faire, il faut conjuguer ce parcours à une expérience d'utilisation des outils d'expertise valides aux yeux des autres experts.

\section{IV.1.b - L'expérience du terrain}

De la sorte, à ce parcours académique, il faut invariablement combiner des expériences de travail variées dans des lieux variés. Ainsi, pour Carl, ancien cadre dans une ONG canadienne de coopération internationale, et spécialiste de la gouvernance organisationnelle, « C'est davantage l'expérience qui me confère cette expertise. J'ai fait mon baccalauréat en pédagogie; on peut dire que j'ai fini dans un domaine que j'ai peu étudié formellement! » Marie renchérit : «À l'ACDI, les experts avaient tous une expérience très concrète du terrain, plusieurs années, dans plus qu'un espace géographique et culturel 
donné (Afrique, Amérique du Sud), pour pouvoir faire une analyse comparative ». De son côté, Audrey, jeune coopérante volontaire en début de carrière, insiste d'ailleurs sur l'importance de la pluralité des expériences culturelles : «[faire de la coopération] c'est faire un travail dans un pays et un contexte culturel différent et dont la principale caractéristique est d'avoir un questionnement du milieu où l'on travaille, pas de faire le travail comme on le fait chez soi. »"

Le nombre de «terrains » effectué devient ainsi un baromètre de l'expérience accumulée et la variété des expériences permet ainsi de départager les formes d'expertise, car selon Carl spécialiste de la gouvernance des organisations de développement international, « il y a des experts internationaux et des experts locaux. » L'expert international apporte le cadre formel du développement international avec lui. Peu importe, sa position éthique et idéologique sur le développement, ce sont les expériences de «terrain» associées aux connaissances académiques réunies par l'expert qui lui permettent d'apporter le recul nécessaire à l'analyse des situations et des enjeux, comme le résume Marie coopérante et spécialiste des questions de genre : «Moi en n’étant pas une femme africaine et en connaissant d'autres droits normatifs par rapport aux femmes je pouvais apporter le recul [aux femmes africaines avec qui je travaillais]. C'est pour ça que je pouvais accompagner ces groupes de femmes comme consultante. »

Concrètement, toutes les expériences de terrain ne sont pas de même valeur, car elles doivent permettre d'acquérir et de développer des compétences précises. Par ailleurs, elles doivent aussi apporter une valeur utile aux gens et groupes sur place (experts locaux, populations, gouvernement, organismes) afin d'amener, selon Leonard, « une compréhension de ce qui serait pratique et de ce qui serait réalisable » comme action pour renforcer le développement. Si le fait d'avoir une formation académique mise en pratique dans une multitude d'expériences de terrain permet de consolider nombre 
de connaissances et d'outils d'analyse précédemment acquis, cela ne peut nous être utile si on ne dispose pas d'une bonne connaissance des modes et des codes de communications appropriés.

\section{IV.1.c - La connaissance des codes du développement international}

Les personnes interrogées évoquent certains « codes » usuels propres à l'échange d'informations en développement international, notamment en contexte africain, avec les ONG et agences canadiennes. Ces « codes » regroupent entre autres, des élément de culture gestionnaires et du jargons bureaucratiques de son employeur tel que l'énumère Gérald, jeune spécialiste des questions de genre en Afrique de l'Ouest; « ...respect des échéances, des critères d'évaluation, des cadres des documents à remplir, les normes de présentations, de révision des documents produits... ». Ainsi dans l'idéal, selon Carl ancien cadre retraité d'une ONG canadienne, « l'expert doit s'adapter aux différentes cultures bureaucratiques européennes, nord-américaines ou des institutions internationales, car ce sont ces entités qui demandent la plupart du temps des évaluations ». Cet expert doit faire usage de la langue occidentale d'usage local, car selon Carl « en Afrique, la langue de travail est souvent la langue de l'expert occidental, ou la langue occidentale dominante de l'ancienne colonie ». Dans le même ordre d'idées, Maurice, expert, anthropologue et pigiste dans la cinquantaine, considère important de connaitre les manières de faire des évaluations de projet ou de programme où l'on doit « amener les gens à dire ce qu'il y a qui ne marche pas. Des fois, que tu le veuilles ou non, à force d'en faire, tu finis par connaitre le domaine ». Les intervenants suggèrent qu'une expérience valable devrait démontrer la capacité d'apprendre et de maitriser plusieurs compétences nécessaires à la bonne intériorisation des « codes » évoqués.

Encore, pour Gérald, les manières de faire une recommandation suivent toujours un cheminement 
avec usage en gradation des modes de communication. On utilise ainsi la communication verbale « formelle et informelle », dans des rencontres « officielles et officieuses ». On suit plus ou moins un processus rigoureux pour transmettre le message voulu jusqu'à l'obtention de la réponse attendue de la part de l'interlocuteur « en y allant par la gradation des trois niveaux; 1) être sur le terrain et démontrer sa présence; 2) discuter des idées officieusement avec les responsables d'un projet; 3) participer à une discussion officielle ou encore produire le bon vieux rapport. » Selon le contexte, on se doit d'utiliser une bonne stratégie pour faire cheminer une recommandation. Cela permet d'influencer le cadre qui détermine la manière dont le terrain sera analysé. Ainsi, Audrey jeune spécialiste des questions de genre en Afrique de l'Ouest manifeste une préférence stratégique dans son choix de code, de canal de communication et des personnes à qui on s'adresse pour s'assurer de l'application de la recommandation :

Lorsqu'on aborde les recommandations, je pratique généralement à l'oral en m'adressant au bas de la chaine de commandement des organisations. Pour qu'une idée reste et soit pratiquée sur le terrain, c'est par elle que l'on doit passer. Si l'on fait une recommandation en passant par les hautes sphères de l'organisation, ça se rend rarement sur le terrain.

La maitrise de ces codes et compétences est démontrée par la polyvalence, soit l'habileté d'un agent à utiliser et à combiner les différents outils académiques (langues, méthodes scientifiques, capacité de rédaction, etc.) acquis à travers le parcours universitaire avec les outils acquis (connaissance des codes bureaucratiques et des coutumes locales) lors de ses expériences concrètes dans une variété de contextes et de conditions. Ancienne fonctionnaire et coopérante Marie, résume en une phrase ce que l'expert en développement doit réunir en plus de son parcours académique : «Je pense que l'ACDI m'a recruté parce qu'elle recherchait ma polyvalence, j'ai travaillé dans plusieurs pays d'Amérique du Sud et d'Afrique. Je parle français, portugais et espagnol. Évidemment, j'avais de l'expérience concrète en développement international. » La réunion de ses critères permet aux 
personnes d'avoir le potentiel d'être un expert légitime. Pour être un expert légitime, ce dernier doit faire des actions lui permettant de maintenir la reconnaissance de son expertise par ses pairs.

\section{IV.1.d - Maintenir la reconnaissance de son expertise}

Selon les personnes interrogées, pour être reconnu comme légitime, le comportement des experts en développement international doit non seulement répondre aux critères mentionnés plus haut, mais doit aussi être reconnu par les pairs. Cette reconnaissance se compose de plusieurs dimensions permettant aux pairs d'identifier l'appartenance d'un expert au champ. Ces dimensions sont, par exemple, l'utilisation des bons jargons techniques, l'association avec d'autres experts, le développement d'une expertise spécifique et la réclamation d'un traitement financier particulier. Ces dimensions seraient essentielles pour que l'on puisse être reconnu comme expert en développement international.

Dans un premier temps, le jargon technique doit être familier et utilisé pour démontrer sa maitrise. Cela permet aux experts de se reconnaître entre eux, mais aussi de se distancier des populations visées par le développement, comme l'évoque Marie : « Le vocabulaire technique utilisé par l'expert accentue cet éloignement, car il casse les discussions franches avec les gens, c'est un piège qui nuit à la relation humaine et à la relation de confiance nécessaire pour bien faire des recommandations. » Pour acquérir le jargon d'expert et s'assurer de le maitriser, il faut fréquenter un milieu d'experts en développement dans lequel on peut s'associer à d'autres experts à travers des firmes de consultants, acquérir des diplômes académiques, faire référence aux termes en vogue ou encore publier des ouvrages. Par exemple, développer « une niche » d'expertise en développement international en publiant des ouvrages permet de valider la reconnaissance de son expertise. Carl nous explique en quoi la publication d'ouvrage permet de devenir expert : «pour être expert, les autres 
doivent te reconnaître comme tel. La reconnaissance par la démarcation (proéminence) par rapport aux autres, par exemple par la publication d'un ouvrage reconnu, aide à devenir expert. »

Si ces pratiques permettent aux experts de se reconnaître entre eux, les institutions qui font appel à l'expertise, ont d'autres critères qu'elles peuvent vérifier plus facilement. Par exemple, certaines institutions vont utiliser le traitement financier élevé exigé par l'expert pour juger de sa crédibilité. L'expert doit percevoir ou réclamer un traitement financier adéquat pour être perçu comme crédible aux yeux des institutions du développement international. Ainsi pour Marie :

On fabrique une super structure élitisante du développement, par exemple si un consultant soumissionne trop bas pour une agence, on ne prendra pas sa candidature parce qu'on a l'impression qu'il est un amateur, un gars du bac, bref qu'il ne fait pas partie de cette élite, du monde du développement.

Cette légitimité et cette crédibilité sont ultimement validées par votre capacité à maintenir de bonnes relations interpersonnelles, car selon Leonard retraité d'une firme de consultant, « ce n'est pas le domaine d'étude des gens qui compte; les qualités personnelles comptent autant que les qualités techniques. Si vous ne pouvez pas obtenir la confiance des gens avec qui vous travaillez, en leur disant exactement ce qui se passe, alors vous ne pouvez pas être efficace. » Pour Carl, il est important de savoir maintenir de bonnes relations personnelles notamment en faisant attention à la culture locale.

Les experts sont constamment appelés à travailler avec des gens ou des experts locaux avec qui ils n'ont jamais travaillé, ils doivent faire preuve d'adaptabilité et de diplomatie. Ils doivent faire état de bonnes qualités dans leurs relations interpersonnelles. Les différences culturelles ne sont pas nécessairement un problème, mais peuvent l'être dans certains cas.

\section{IV.1.e - Être actif en développement international}

La crédibilité et la légitimité de l'expertise passent aussi par une démonstration de l'activité dans le champ de l'expertise en développement international. Seul un expert actif peut espérer être reconnu 
crédible et légitime. L'inactivité prolongée démontre un éloignement du contexte ne permettant plus de se prévaloir du statut d'expert. Comme le souligne Carl « je suis probablement un expert, un peu rouillé parce que je ne suis pas « au courant », car je n'ai pas été à l'extérieur du pays depuis trois ans... Je fus un expert, mais plus maintenant! $»^{12}$ Pour être expert, il faut surtout être « au courant » de ce qui se passe en développement international, soit : constamment « actualiser» ses pratiques pour être «à la fine pointe du savoir » académique et pratique, connaitre les auteurs, les théories et les pratiques à la mode et prisés par les bailleurs de fonds, mais aussi connaitre l'actualité et le contexte du terrain dans lequel on est appelé à évoluer. Ainsi, pour Gérald, il est clair qu'« il faut maintenir un équilibre entre la pratique concrète du développement et la consommation de savoir scientifique et technique. Dès que ces conditions ne sont pas réunies, on perd le titre d'expert. » Être actif permet ainsi non seulement de maintenir ses habiletés, mais aussi d'en apprendre de nouvelles comme l'expose Leonard :

$\mathrm{Au}$ début, j'ai surtout utilisé ma formation en économie, mais plus j'ai été impliqué dans les évaluations, plus j'ai dû apprendre par moi-même. J'ai développé mes principales expertises de façon autodidacte en apprenant sur le sujet, en utilisant des outils et en donnant des formations. La fréquentation des cercles académiques par la lecture de ses publications m'a fourni plusieurs outils d'évaluation.

L'expert est en mesure de démontrer qu'en plus de posséder les expériences, formations et maitrise de codes nécessaires, il se montre actif dans le champ de l'expertise en développement tant dans la sphère de production et d'acquisition de savoir que dans la sphère d'application de l'expertise. Or, pour être considéré comme légitime, l'expert se doit de s'assurer que tout ce qu'il a acquis et appris est mis en pratique dans l'optique de démontrer la valeur et la pertinence du développement.

12 Notre tradcution: "I'm probably an expert, I'm a bit rusty because I'm not currently "au courant". I haven't been outside the country for three years now... I used to be an expert! » 


\section{IV.1.f - $\underline{\text { Avoir recours à la méthode scientifique }}$}

L'expert doit être en mesure d'utiliser les connaissances et les outils analytiques appropriés pour sanctionner le développement d'une autorité scientifique destinée à mettre en valeur l'idée de développement. Elle lui confère un sérieux qui lui permet de passer le test de sa pertinence. En ce sens, pour Maurice, consultant indépendant spécialisé en suivi et évaluation de projet, il faut dépasser le stade de l'opinion sur le développement international et mettre en lumière le fait que l'expert est essentiel ainsi que ses compétences pour faire du développement international;

«Est-ce que les experts peuvent nuire au développement international? » Non, c'est le contraire. C'est les gens qui ne connaissent pas ça [le] développement international [qui peuvent lui nuire]. Tout le monde connait ça, tout le monde a le droit à son opinion là-dessus [...] c'est le manque d'experts qui doit être vu comme le plus dangereux pour moi, pour les pays en développement, plutôt que le contraire.

Ainsi, on évite de faire des jugements hâtifs et de démontrer une méconnaissance du milieu, soit une ignorance des limites de ses connaissances comme l'expose cette critique du ministre Fantino ${ }^{13}$ par Maurice :

Tu peux pas aller dans un pays en voie de développement, être un ministre de la coopération internationale puis dire: "on peut pas travailler ici, c'est pas propre." Tu sais quand il est allé en Haïti... ... et que sa grosse conclusion après avoir visité un bidonville c'est que c'était pas propre. Fantino, c'est après ça qu'il a dit qu'il voulait couper l'aide à Haïti... ... parce que c'était pas propre... ... un bidonville propre j'en vois pas trop.

En prononçant de telles paroles, le ministre se discrédite aux yeux de Maurice qui refuse de le reconnaître comme un acteur positif du développement. C'est la méconnaissance des réalités de base du développement et le recours aux opinions pour identifier les occasions de mettre le développement en

13 Au moment de l'entretien, Julian Fantino, député de la circonscription de Vaughan en Ontario (29 novembre 2010 - 21 octobre 2015) occupait le poste de Ministre de la coopération internationale dans le gouvernement conservateur du premier ministre Stephen Harper. Au sein de ce gouvernement, le ministre était directement responsable de l'ACDI. Il a occupé ce poste du 4 juillet 2012 au 15 juillet 2013 peu après l'entretien. 
valeur qui est dérangeant. Conséquemment, pour Maurice, cette personne nuit au développement parce qu'elle en évacue le caractère scientifique. L'expertise est donc un caractère que l'on construit dans le temps à partir de son parcours académique, de ses expériences dans le domaine du développement, dans lequel on doit nécessairement faire un traitement «scientifique » de l'information qui sert à légitimer ses actions. Maurice souligne bien cette réalité :

Le développement international, il faut que ce soit traité comme une science. T'as des experts làdedans, t'as un aspect économique qui est très important. Tu as des études à faire, tu as des recherches à faire, tu ne peux pas t'improviser comme ça [et dire :] «et bien moi, selon moi, on devrait faire ci, on devrait faire ça, c'est fini [ce temps-là], c'est plus compliqué que ça [aujourd'hui].

Nous savons maintenant que les personnes rencontrées estiment que l'on devient expert en prenant part à différentes activités qui permettent d'acquérir des habiletés et des connaissances, notamment, pendant le parcours académique et lors des expériences «terrain» dans des projets de développement par exemple. On peut réussir à mettre ces acquis en valeur dans le champ du développement international notamment grâce à une bonne connaissance des codes sociaux associés au travail en développement tels les termes d'usage dans les agences et les ONG, ou encore par la connaissance des procédures bureaucratiques. Finalement, cumuler le fait de se montrer actif en développement et avoir recours à la méthode scientifique permet d'obtenir la reconnaissance nécessaire pour revendiquer le statut d'expert légitime. Or, si ces informations sont importantes pour bien comprendre d'où vient l'expert en développement international, elles n'éclairent que partiellement sur l'utilité de l'expertise dans ce domaine. Celle-ci sera abordée dans la prochaine section de ce chapitre. .

\section{IV.2 - À quoi sert l'expert}

Pour être praticable et légitime aux yeux des experts, l'expertise doit être demandée par l'une des 
parties prenantes (donateur, intermédiaire, bénéficiaires, etc.) à un projet de développement international. Ainsi, pour Audrey, « l'appel à l'expertise ou l'envoi d'experts sous-entend que quelqu'un, quelque part, a estimé qu'il y avait un manque de connaissance lié au développement dans les communautés visées. » Pour d'autres, c'est la situation qui exige la présence d'experts comme le résume Maurice pour le cas du Burkina Faso « ...ça ne marche pas très bien, il y a des problèmes, autrement on ne serait pas là-bas. » Suite à cette demande d'expertise et selon la forme qu'il juge nécessaire, chaque expert détient une approche et un style favorisant la transmission de ses connaissances. Chaque style de pratique de l'expertise privilégie une forme de connaissances à apporter, une manière de le faire qui lui est propre et un type de relations différentes avec la communauté/l'organisme ciblé. Ce sont des extrêmes vers lesquels chaque expert tend dans sa pratique. Ainsi la plupart des experts orientent leur pratique pour répondre à un besoin éventuellement identifié par les agences ou les organismes sur le terrain en matière de développement, par exemple : un besoin de connaissances, de ressources, de contacts, de manières de s'organiser, etc.. Selon les besoins et les capacités de l'expert, sont apportées des solutions, des interrogations, des ressources, des traductions au moyen d'un style d'expertise particulier. Parmi les styles énumérés par les personnes rencontrées, on notera sur un premier axe deux styles qui s'opposent dans la manière d'apporter la connaissance : le « solutionneur » qui apporte des solutions et son opposé l'« intellectuel» qui apporte des questionnements. Sur un autre axe traitant plutôt de la compréhension interculturelle, on trouvera le « traducteur » permettant aux personnes dese comprendre d'un univers culturel à l'autre et son opposé, la « personne-ressource » qui aiguille les organisations à travers les méandres de l'industrie du développement.

\section{IV.2.a - Le solutionneur}

Le solutionneur est vu par plusieurs comme celui qui apporte des solutions à un problème dans 
un projet ou une situation propice à une activité de développement, une approche que la plupart des experts observent avec méfiance. Pour Audrey, coopérante spécialiste de la communication et de l'intervention communautaire, le solutionneur travaille souvent seul, car « il a une approche individuelle; en vase clos. » Pour Audrey, cet expert prescrit des actions et se campe dans le discours technique, car « il est là pour apporter des réponses, il adapte moins son travail et se rend rarement à un niveau facilement compréhensible pour le non-expert. » C'est une approche qui comporte ses risques sur le plan éthique, selon Marie : «D'abord, si on arrive dans un autre pays avec un livre de prescription, en disant, nous dans notre pays, on fait ça comme ça et comme ça, on peut nuire parce qu'on arrive à vouloir mettre tout le monde dans la pensée unique, c'est une façon de faire qui peut nuire au processus [de développement]. »

Pour imposer ses cadres, le solutionneur fonde son intervention sur l'autorité que lui confère son titre, ses fréquentations, son lieu d'origine et sa discipline d'appartenance. Ainsi, l'anthropologue et consultant Maurice dresse la caricature d'une situation hypothétique où l'on doit faire appel à un tel type d'expert :

Quand tu veux évaluer un projet en santé, si tu cherches un évaluateur, ne va pas chercher un médecin parce que le médecin il arrive là avec le syndrome du beau-frère du bon Dieu; tu sais il sauve des vies, hein! Et bien lui, il va aller dans un pays en voie de développement, tu sais «dans ces pays-là » parce que « ces gens-là peuvent pas connaitre ça, ils ne viennent pas du Canada, ils n’ont pas étudié à McGill... » Lui le médecin, il connait ça mieux que tout le monde.

Dans sa description péjorative, cet expert que l'on dénonce cherche à être en permanence audessus de la mêlée, ce qui le met dans une position permanente d'enseignement de son savoir, une situation que le statut d'expert contribue à cadrer selon Maurice, car « tu sais, c'est dur de résister à ne pas enseigner ton savoir. [Les experts] ne sont peut-être pas tous de même, mais disons que j'en ai vu très souvent que c'était ça [leur problème]. » Les personnes rencontrées placent ce style de pratique de 
l'expertise en opposition avec celui qui apporte des questions plutôt que des solutions.

\section{IV.2.b - L'intellectuel}

À l'opposé du solutionneur, Audrey nous décrit un autre style d'experts, l'intellectuel qui « est là pour apporter des questions pour susciter des réflexions sur le projet pour lequel il travaille ». La collaboration et le dialogue sont au centre de ses préoccupations, car « il travaille de façon collaborative afin d'aider un groupe à se remettre en question pour faire émerger de nouvelles solutions et de nouvelles façons d'envisager un problème. » Pour Carl ancien cadre d'une ONG canadienne, « certains [experts] sont des auteurs publiés et sont plutôt connus dans le domaine en raison de leur proéminence $\gg{ }^{14}$ parce qu'ils participent aux débats publics sur le développement et publient des ouvrages. On trouve ici un style d'expertise plus caractérisé par une attirance pour les interrogations et la recherche d'informations plutôt qu'un aspect prescriptif.

Or pour plusieurs experts, la tension entre la réflexion amenée par les questions et l'action amenée par les solutions n'est pas le seul élément qui nécessite l'apport d'une expertise. D'un autre côté, les interlocuteurs ont un besoin de se faire comprendre et d'utiliser les bons outils.

\section{IV.2.c - Le traducteur-facilitateur}

Sur l'axe de la compréhension, on trouve à une première extrémité, le traducteur-facilitateur. Audrey voit ce style d'expert comme celui qui « [...] est là pour faire émerger des idées, pour faire baisser les tensions interculturelles, proposer un nouveau regard et d'autres avenues de solutions. » Par exemple, Maurice un anthropologue spécialiste du développement international se présentant comme

14 Notre traduction: "Some of them are published authors and they are well known and expert because of their proeminence » 
un «traducteur» œuvrera pour faire le pont entre deux groupes aux origines et aux perspectives culturelles différentes qui cherchent à se comprendre pour former des partenariats :

Bien moi je suis là pour expliquer aux deux, souvent pour compléter l'information aux Canadiens, pour leur dire la situation est la suivante OK... ... Voir aussi la pertinence, parce que souvent c'est très dur de transférer une technologie, ils n'ont pas la capacité économique pour la recevoir ou pour la maintenir. Fait que tu essaies, t'expliques aux gens des pays en développement c'est quoi le contexte ici, tu t'assures que le contexte est bien compris, et la même chose sur les demandes, tu fais le suivi de ça et t'en arrives en avoir un projet qu'eux développent, mais que toi tu fais le suivi par la suite. Tu les as mis ensemble, tu les as aidés à discuter puis ensuite t'es responsable de faire le suivi : quand t'es agent de suivi.

De cette façon, pour la coopérante et spécialiste de la communication sociale Audrey, il n'est pas prioritaire que la connaissance soit amenée par l'expert : « il n'est pas important que les idées viennent de cette personne, mais plutôt que sa présence favorise l'émergence de nouvelles idées. » Ainsi dans une situation d'évaluation, Maurice décrit son rôle et déclare : « t'es pas là pour vendre ta salade, pour dire que t'es meilleur qu'eux autres : t'es là pour les aider à régler les problèmes. C'est ça un évaluateur. J'essaie de vendre cette idée souvent, puis les résultats sont bons quand j'interviens. Pourquoi? Parce que j'ai pas mis le trouble. » Son rôle devient ainsi davantage celui qui aide les bailleurs de fonds et bénéficiaires à se comprendre, à saisir les nuances et à s'entendre en arrivant sur une définition « commune » par rapport à l'avancement d'un projet vers les attentes. Dans d'autres situations, il est plus important de connaitre les ressources disponibles qui deviennent importantes pour un organisme. Dès lorscomprendre la manière de fonctionner de l'industrie du développement devient plus utile. Certains développent une expertise en devenant une ressource grâce aux multiples expériences dont ils peuvent faire bénéficier leurs employeurs et en aidant les bénéficiaires à comprendre l'industrie du développement. 


\section{IV.2.d - La personne ressource}

Les «personnes-ressources », «agents de liaison» se spécialisent dans l'apport du bénéfice de ses expériences pour aider les gens à comprendre les problématiques auxquelles ils font face: pour Audrey, «Cette personne possède un important bagage d'expérience sur un sujet particulier et une formation générale. [Cet expert] s'assure de favoriser une approche participative d'ouverture à la collaboration pour aborder les problématiques rencontrées. » Ainsi, ce transfert d'expérience s'avère nécessaire pour Carl, car les conceptions occidentales de l'aide et de la manière dont les ressources doivent être administrées sont différentes parce que,

L'ONG est un concept profondément occidental, souvent les problèmes rencontrés vont être d'ordre organisationnel et financier. Quand on le consulte pour son expertise, l'expert identifie les lacunes organisationnelles et il recommande les actions que d'autres organismes ayant vécu un problème similaire ont implantées. Pour l'aspect financier, il peut favoriser la mise en contact avec des gens qui « savent comment faire ».

La personne-ressource est ainsi amenée à indiquer où sont les ressources appropriées grâce à son expérience, à faire part de ses apprentissages et à indiquer aux agents comment ils peuvent faire leur chemin à travers l'industrie du développement et en tirer ce dont ils ont besoin.

Les experts apportent au développement différentes formes de connaissances et différentes catégories de ressources et de relations selon des styles qui tendent à être assez définis. Les solutionneurs règlent les problèmes grâce à l'application de leurs solutions préparées alors que les intellectuels remettent en question les aprioris idéologiques et restituent une perspective élargie des problématisations possibles de la situation de développement qui leur est confié. Lese traducteursfacilitateurs s'assurent que tous se comprennent alors qu'à l'opposé les personnes-ressources aiguillent vers les pratiques et moyens valorisés en développement. Cet ensemble de positions n'est pas statique, 
il constitue un ensemble d'extrêmes à la fois de préférences et de compétences que l'on reconnaît aux experts. Or, selon les experts rencontrés, l'efficacité d'une approche ainsi que la manière de déterminer l'approche la plus pertinente pour une situation donnée sont influencées par l'ambiance dans laquelle l'expert pratique son expertise, une ambiance qui varie, mais où certaines constantes s'observent et seront examinées dans la prochaine section.

\section{IV.3 - Ambiance de pratique de l'expertise en développement international}

Nous avons désormais identifié les prérequis et les styles d'expertise le plus souvent utilisés dans ce champ selon ce qui est estimé par la plupart des personnes rencontrées. Il importe désormais d'identifier dans quelles conditions s'effectue ce travail. Dans cette section, nous avons cherché à mettre en lumière les propos qui expliquent les conditions dans lesquelles les experts estiment accomplir ou non celui-ci, en les laissant décrire ce qui les freine ou ce qui fait leur succès. Deux thématiques principales en sont ressorties, soit l'objectivité dont ils doivent faire preuve et les qualifications nécessaires à acquérir pour être expert.

\section{IV.3.a - L'objectivité : entre bureaucratie et politique}

Selon les personnes interrogées, le développement se fait dans un cadre exclusivement gestionnaire et comptable. Tel que le développement nous a été décrit, tout y est comptabilisé et doit avoir une valeur monétaire démontrable par des faits. De ce point de vue, tout investissement doit produire des résultats optimisant à la fois une efficacité économique pour les ressources mises en place et une efficience économique dans la mobilisation des agents. Pour s'assurer de cela, au Canada, les projets sont soumis par l'ACDI à l'évaluation d'un expert selon une grille de critères. Or, les critères d'évaluation se trouvent constamment remis en question pour valoriser certains acteurs plutôt que 
d'autres. En ce sens, les données sont souvent présentées de façon complaisante afin de montrer de bons résultats. Par exemple, Maurice évaluateur à son compte, évoque une discussion à propos de la façon dont on lui a demandé indirectement de cadrer une évaluation :

«Bien là si t'as des plus grosses parcelles, t'as moins de participants. » «Puis nous autres on veut montrer qu'on a beaucoup de [participants] parce que l'ACDI comme toutes les agences [demande] : « on a combien de bénéficiaires, ça nous en prend $10000 \ldots \ldots \ldots$.... On va faire des plus petites parcelles... ...... Tout le monde veut démontrer qu'avec peu [on fait beaucoup]. Tu sais l'efficacité puis l'efficience, ça là, ça tue, c'est mortel, c'est pas réaliste, les attentes sont trop grandes pour [les ressources qu'on mobilise].

Pour Audrey, experte en communication sociale et coopérante, c'est le choix des critères d'évaluation qui pose problème, car ces derniers répondent à des exigences étrangères aux bénéficiaires et présentent un fait avec un lien apparent avec le développement comme un gage de celui-ci : «Les fonds sont remplacés par des coopérants, le tout dans une logique comptable qui voit l'envoi de coopérants comme moins coûteux parce qu'elle prodigue de la connaissance à moindre coût et démontre par des faits une utilisation de fonds légitime. »

Cette comptabilisation et ce cadre gestionnaire entrainent une pression sur les experts et les autres travailleurs du développement. Pour survivre sur le plan professionnel, Leonard et Audrey nous indiquent qu' ils ont des obligations, soit « ramener des contrats, payer des factures, accumuler des expériences, des travaux » auxquelles s'ajoute pour Audrey, la nécessité « d'accumuler les diplômes et les certifications ». Ainsi les experts doivent pratiquer des activités qui ne sont pas toutes rémunérées tout en « ramenant de la nourriture sur la table » pour reprendre l'expression de Maurice, anthropologue dans la mi-cinquantaine. Sur le plan individuel, les experts doivent subvenir à leurs besoins « de base » pour maintenir leur statut. Sur le plan organisationnel, cette réalité est encore plus marquée pour les firmes de consultants : « les firmes de consultants sont des entités d'affaires; elles vont se nourrir de 
toute forme de travail qu'elles vont trouver » note Carl. Tout juste retraité d'une firme d'expertsconsultants en développement international, Leonard renchérit sur les conséquences éthiques de l'organisation du travail en firme :

Les entités d'affaires doivent payer les factures, alors elles doivent toujours ramener des contrats, ce qui veut dire qu'elles ne peuvent pas se permettre d'être difficiles dans les contrats qu'elles font. Elles ne peuvent pas choisir que leurs sujets de prédilection ou seulement des organismes qu'elles aiment ou des missions dans lesquelles elles croient. Elles sont "froides", en ce sens que tout est une occasion de travail pour ramener de l'argent qu'on ait la capacité de le faire avec qualité ou non : dans une firme on tente de maximiser les revenus.

On comprend de ces propos que les experts du développement sont constamment sous pression pour produire des résultats. Cependant, ils nous éclairent moins sur la provenance de cette pression, alors que la temporalité des projets et l'influence des élus sur le champ bureaucratique nous renseignent.

\section{Une fenêtre d'action " cyclique " et courte}

Le cadre gestionnaire d'un projet de développement instaure une temporalité cyclique de l'évaluation des projets qui transfère la pression sur l'expert-consultant. Ainsi, les périodes d'évaluation sont volontairement courtes, car obtenir des « résultats » rapidement est priorisé par rapport à une approche à long terme, c'est-à-dire que la finalité comptable est privilégiée face à toute autre conception du développement. Ainsi, selon Maurice, «Certaines [agences ou organisations] veulent avoir des projets plus courts pour pouvoir couper les fonds si cela ne fonctionne pas comme elles l'entendent ou si encore des raisons politiques se présentaient. » En effet, la plupart des projets et des contrats en développement international sont de courte durée, en particulier pour les consultants : « la durée des contrats de consultation varie. Des fois c'est huit jours, quinze jours, deux mois, d'autres six mois, pour être franche, certaines années c'était seulement trois mois par année, alors j'avais du temps 
pour faire autre chose », nous dévoile Marie.

Ce cadrage des opérations d'expertise et de vérification des projets est destiné à garantir l'utilisation des « meilleures pratiques » pour ne pas répéter les mêmes erreurs en imposant une période d'évaluation courte. On souhaite ainsi « dépolitiser » le processus de décision, cependant on ouvre la porte à l'implication des décideurs politiques, ce que Frank, économiste spécialiste de l'environnement pour le compte de l'État canadien, dénonce :

C'est incroyable le nombre de décisions qui se font sans analyse et sans « evidence based decision making ». Il y a des décisions qui se font pour des raisons politiques et d'autres qui se font pour des raisons peut-être plus stratégiques sans nécessairement regarder ce que disent les académiciens... En absence de bonne analyse en terme de politiques publiques [en terme de] ce qui fonctionne ou pas [de] ce qu'on a essayé et qui a été désastreux. C'est incroyable le nombre d'erreurs et de trucs que l'on fait, qu'on sait que ça ne fonctionne pas. C'est comme si les leçons apprises sont pas bien résumées ou pas bien transmises aux décideurs, ou elle est transmise et ignorée.

Ce type de fonctionnement est selon lui une aberration, car on ne se donne pas le temps d'avoir des résultats probants et on ne permet pas d'utiliser ce qui est déjà désigné par les milieux intellectuels du développement comme « les meilleures pratiques ». Le cadre de l'action cyclique est possiblement un carcan qui limite l'action des experts et permet à d'autres de restreindre ce qui peut en sortir par souci de contrôle. On se trouve alors dans une situation où la lutte au sein du champ bureaucratique en vient à influencer directement ce que l'expert peut faire ou ne pas faire.

\section{L'influence des élus sur le champ bureaucratique}

La présence des cycles tend à s'expliquer par le besoin des fonctionnaires de se prémunir face à un projet qui tournerait mal. À cette échelle, on souhaite réduire les risques d'échec et de fraude en favorisant la centralisation des décisions au sommet de la hiérarchie décisionnelle. Ce cadre est destiné 
à garantir l'utilisation des « meilleures pratiques » pour ne pas répéter les mêmes erreurs. La décision qui est appliquée est celle qui est décidée par ceux qui occupent les postes de direction au sommet de l'État canadien. Ainsi, la centralisation des décisions de l'État et le cadre exécutoire fourni à ses propres experts peuvent parfois se trouver en conflit avec le cadre d'objectivité censé réguler le processus idéal d'expertise en développement international. Maurice propose une caricature de celui que l'on appelle le fonctionnaire :

L'ACDI qu'est-ce que tu veux qu'ils disent quand il y a un changement impertinent aux yeux des experts qui est proposé par les élus responsables de l'agence? On te répond simplement «moi en tant que fonctionnaire, je suis là pour servir mon ministre. Si je veux changer de quoi, j'ai rien qu'à me faire élire. Ça règle la question passons à autre chose. » [...] Là on en est rendu au partenariat avec le secteur privé et l'aide humanitaire... ... Tout à coup, de même: claquement de doigts; on vient de changer ça. Il reste quoi dans les pays où il n'y a pas de partenariat avec le privé, où il n'y a pas de mine?

Elle reste très proche de ce que le fonctionnaire Frank lui-même décrit à propos de son rôle : « ta job comme fonctionnaire, c'est d'apporter le meilleur conseil possible même si ce n'est pas le message que les élus veulent entendre. Mais une fois que la décision est prise par les élus, ta job c'est de faire la mise en œuvre la plus efficiente et le plus efficace possible. »

On comprend alors que la pression et le pouvoir des élus de même que l'évaluation cyclique et rapide des projets se conjuguent pour maintenir une pression constante sur les travailleurs du développement. Au-delà du romantisme qui accompagne souvent l'idée de développement, tout comme à la fonction publique, l'industrie du développement exige d'avoir une compétence scientifique marquée pour se qualifier d'expert. 


\section{IV.3.b - Les qualifications de l'expert en développement}

En effet, l'État canadien et les employeurs demandent des consultants en développement international des qualifications similaires et la capacité d'être objectif grâce à l'expérience scientifique. C'est donc un secteur qui fait l'objet d'une professionnalisation marquée et tendue. Cette professionnalisation se manifeste par exemple à travers le souhait d'établir une qualification technique uniformisée en développement international. Selon Maurice spécialiste de l'Afrique et des Caraïbes, ce souhait est justifié:

Ça prend quelqu'un, [un expert], pour leur dire que non ça fonctionne pas comme ça, le développement il y a une façon de le faire ça, mais ça, c'est moins là. Il devrait y avoir une reconnaissance des bases techniques développées qu'il faut suivre pour faire du développement et de la coopération. Comme c'est là, on improvise beaucoup, car il n'y a pas de vision continue du développement au Canada.

D'autres s'inquiètent de cette professionnalisation et critiquent ses possibles dérives à travers une course aux qualifications techniques. Selon Audrey, par exemple, elle entraine une tension dans le domaine entre qualification par la formation et qualification par l'expérience :

J'exprime un doute par rapport aux formations qui apparaissent actuellement en coopération internationale, et développement, etc. À mon sens, elles ne sont pertinentes que si elles donnent des outils pour interpréter les situations. On ne peut pas sortir de ces formations et s'estimer " expert». Il faut faire attention aux formations, elles donnent l'impression de connaitre ce que l'on fait. On y omet souvent les compétences personnelles et les qualités relationnelles nécessaires en contexte interculturel.

Pour Audrey, la professionnalisation n'est qu'une facette de la domination de la culture bureaucratique du développement. En plus d'engendrer la professionnalisation, en mettant l'accent sur les «meilleures pratiques », la culture bureaucratique marginalise les approches divergentes basées sur la relation, l'intervention et l'insertion sur « le terrain » sous couvert de neutralité, au profit d'approches 
qui s'accommodent davantage de l'évaluation quantitative que qualitative comme du postulat de la rationalité des agents :

J'ai comme l'impression que des gens comme moi, qui souhaitent sortir de la temporalité occidentale, qui souhaitent axer l'intervention sur l'échange, sont de plus en plus rares dans notre domaine, que ces visions du développement sont en train de disparaître [...] J'éprouve un isolement et un sentiment de rejet du milieu face à mes pratiques hétérodoxes qui ne répondent pas aux impératifs des courants idéologiques dominants.

Pour d'autres, en plus d'avoir des qualités techniques reconnues, il faut surtout s'assurer de polir ses relations personnelles pour maintenir son statut d'expert qualifié en développement international. Maintenir la confiance de ses partenaires de travail grâce à l'étalement de ses bonnes qualités personnelles devient ici très important pour solidifier le lien des organisations, de l'avis de Leonard : «Ce n'est pas le domaine d'étude des gens qui compte, les qualités personnelles comptent autant que les qualités techniques. Si vous ne pouvez pas obtenir la confiance des gens avec qui vous travaillez, de leur dire exactement ce qui se passe alors vous ne pouvez pas être efficace. » Pour Carl, un expert doit être en mesure de travailler avec tout type de personne et ses qualités interpersonnelles sont primordiales pour pouvoir transmettre son expertise, car :

Les experts sont constamment appelés à travailler avec des gens ou des experts locaux avec qui ils n'ont jamais travaillé, ils doivent faire preuve d'adaptabilité et de diplomatie. Ils doivent faire état de bonnes qualités dans leurs relations interpersonnelles. Les différences culturelles ne sont pas nécessairement un problème, mais peuvent l'être dans certains cas.

La qualification à communiquer une expertise reconnue devient alors aussi importante que la socialisation entre experts. Il importe alors de savoir où et comment les experts socialisent afin d'en comprendre le processus. 


\section{Les lieux de socialisation de l'expert}

Pour tous les experts consultés, il existe une distinction à faire entre les mondes dans lesquels l'expert évolue et socialise. En quelque sorte, les experts vivent dans des mondes parallèles. D’un côté, il y a le monde « à développer » où l'essentiel des actions de développement aboutit et de l'autre, les «mondes parallèles» des «développés/expatriés/blanc» où l'on retourne après le travail. Marie spécialiste de la question genre dans la soixantaine et étudiante aux cycles supérieurs en sciences sociales observe le phénomène suivant :

Les consultants classiques se retrouvent souvent aux mêmes endroits sur le terrain, dans les mêmes établissements, restaurants, bars, hôtels coupés des populations. Surtout en Afrique, ces comportements sont encouragés par les ambassades qui y organisent fêtes, banquets, réunions et soirées de rencontre. C'est une structure entraînante par le luxe qu'elle affiche. Cela crée un monde parallèle.

Selon Marie, ce «monde parallèle » voit son accès filtré principalement à travers la rémunération.

Ce qui fait que les experts se sentent experts, c'est le traitement financier de son travail [très supérieur à celui des autres travailleurs en développement] : ce traitement met de la distance entre l'évaluation et le terrain, mais il rend difficile pour l'expert d'avoir de l'empathie; trop de détachement empêche de connaitre et comprendre la réalité du terrain.

L'accès à une bonne rémunération est lui-même l'objet d'un cadrage à travers l'espace social de provenance de l'expert. Sa provenance lui procure, lui permet ou l'enjoint à exiger un traitement financier différencié selon ses origines et son parcours académique. Ainsi, Gérald spécialiste du genre en Afrique de l'Ouest approchant la trentaine expose le traitement différencié selon l'origine culturelle où « un coopérant canadien fait le salaire moyen par mois de quatre ou cinq coopérants burkinabés [...]

On lui a donné 45000 dollars sur deux ans on va dire? À ce prix-là, on aurait pu engager dix 
Burkinabés pour faire la même bricole puis le projet aurait été dix fois plus ambitieux, dix fois plus gros. » Ce traitement financier permet d'avoir accès au niveau de vie des expatriés. Ce niveau de vie est associé à des lieux dont le coût prohibitif augmente la césure sociale ressentie et cela contribue à renforcer le sentiment de réelle possession de l'expertise chez l'expert.

Si l'on connait maintenant où les experts interagissent, quelles pressions ils subissent dans quel cadre et ce qu'on exige d'eux, on peut maintenant comprendre ce que le travail de l'expert est censé apporter selon sa situation propre, comme fonctionnaire, coopérant ou comme consultant à son compte.

\section{Des institutions et des organisations canadiennes de développement international sous tension}

Si l'on comprend que les agences canadiennes influencent le développement tel qu'appliqué par les organismes canadiens et de ce fait le travail de tous les experts canadiens, on peut ainsi mieux saisir le rôle de ces derniers. Dans cette optique, l'expert est appelé à trancher dans les débats face à une littérature divergente. Pour Frank, la tâche de l'expert est claire :

Quand tu fais une recommandation, tu vas voir s'il y a un consensus dans la littérature, s'il y a plein d'académiciens, de «think tank» qui convergent dans leur opinion, s'il y a une théorie prépondérante là-dessus, c'est relativement simple quand il y a un consensus scientifique, c'est beaucoup moins évident quand il n'y a pas de consensus au niveau académique.

Or, ce rôle devient périlleux en particulier en raison du manque de consensus académique sur plusieurs enjeux du développement international. Carl résume ainsi le paradoxe du rôle de l'expert en développement dans l'amélioration des conditions des communautés visées par le développement :

La contribution de l'expert à la réduction de la pauvreté dépend de la compréhension que l'expert a de la façon qu'on doit s'y prendre pour réduire la pauvreté. Dans le domaine du développement, il y a comme vous le savez une multitude de points de vue différents sur la chose. La conception du 
monde de l'expert influence beaucoup la façon dont l'expertise est pratiquée, elle le rend humble ou arrogant. L'expert qui reconnaît ses limites est plus utile que celui qui se laisse consumer par l'importance de son expertise.

Toutefois si le rôle attendu des experts varie peu, le contexte quant à lui peut avoir une influence importante sur les experts et les expertises dominantes quant à elles peuvent créer une ambiance particulière. En effet, depuis l'arrivée au pouvoir d'un gouvernement conservateur à la tête de l'État canadien en 2006, il y a un sentiment, tant chez certains experts de la fonction publique que chez certains consultants-experts rencontrés, de tension par rapport à l'instauration d'un mécanisme de décision normalisé par l'idéologie néolibérale et néoconservatrice. Ainsi, dans la fonction publique, on note une importante dévalorisation de l'expertise vers une valorisation de ce que le fonctionnaire et économiste approchant la quarantaine Frank qualifie de « raisons idéologiques » :

Comme fonctionnaire, les choses ont évolué beaucoup depuis que je suis arrivé dans la fonction publique en 2000. La valeur... ... tu te sens beaucoup moins valorisé comme analyste de politique publique qu'au début des années 2000. Il y a beaucoup plus de décision qui sont prise malgré les recommandations, ou sans même qu'il y ait de recommandation ou que la fonction publique se soit fait demander son opinion, sans que les devoirs ne soient faits nécessairement, donc des décisions qui sont prises pour des raisons idéologiques sans que ce soit du evidence-based decision-making.

Pour le consultant indépendant Maurice, il s'agit d'un « manque de reconnaissance au niveau gouvernemental du fait que le développement est un champ d'expertise [...] tu ne peux pas prendre des décisions strictement politiques quand tu fais du développement, quand tu veux avoir des résultats. »

Du côté des coopérants, le modèle des ONG est en crise. Depuis l'arrivée des conservateurs au pouvoir, il y a une baisse du financement dans un cadre où la source principale de subventions et de données sur le développement est l'État. Pour Maurice, l'ACDI joue un rôle central dans le cadrage de leurs activités, mais aussi dans leurs choix d'activités : 
Ici, t'as l'ACDI, c'est la source de données, les grandes organisations, la majorité de leurs fonds viennent de l'ACDI. Il y a des collectes et des levées de fonds, mais ... maintenant, c'est de plus en plus important parce que l'ACDI finance de moins en moins les ONG, mais les ONG disparaissent aussi.

Cette baisse de financement a un impact à plusieurs niveaux sur les activités de développement. Ainsi, elle se répercute sur les ONG partenaires situés dans les pays visés par le développement. Selon la coopérante Audrey, « Les organismes sont en état de dépendance et souvent, l'arrivée d'un coopérant que les agences perçoivent comme un gage de développement à moindre coût est perçue [par les organisme locaux] comme un échec de financement, et un manque de matériel pour faire aboutir les projets ». Cet impact se voit aussi dans la pratique de l'expertise de tous les jours et Carl ancien cadre d'ONG aujourd'hui à la retraite est catégorique à ce propos : « Les experts disparaissent actuellement, car il y a moins d'évaluation du travail des ONG par l'ACDI; il y a donc moins de chance d'acquérir de l'expérience, donc moins de gens qui combinent savoir académique et expérience du terrain. »

Le modèle actuel des ONG de développement est en crise actuellement en raison de changement dans les critères à atteindre pour être financé. Depuis l'arrivée des conservateurs à la tête de l'État canadien, il y a un changement drastique de ceux servant à déterminer ce qui est un interlocuteur finançable pour l'ACDI. À ses yeux, ces critères sont sciemment cachés alors que parallèlement il y a des rejets de demande en raison de positions politiques des soumissionnaires. Cette façon de faire a un impact important et perturbe toute la communauté du développement, une perturbation dont l'ambiance peut être résumée par les explications de Carl :

L'ACDI en voulant devenir plus efficace et pour réduire ses coûts, s'était mis à reconnaître des organismes comme compétents et à leur attribuer des enveloppes de financement, ce qui donnait plus de liberté aux ONG pour gérer ces fonds. On avait donc des gouvernements qui avaient confiance dans les ONG, un climat différent du climat actuel. Alors aujourd'hui ces organisations doivent toutes faire des soumissions et quand on décide qui va recevoir, il n'y a pas de transparence dans le processus, on ne dit pas au rejeté pourquoi ils ne se qualifient pas pour du financement, 
d'autres perdent leur financement pour des raisons politiques tel que CAIROS ou encore

Développement et paix. Pour ces raisons il y a un froid actuellement dans la communauté du développement canadien.

\section{IV.4 - Les situations d'expertise}

Dans une telle ambiance, les experts ne sont pas sans réfléchir à propos des situations rencontrées dans le cadre de leur travail. Dans cette section, nous relatons les extraits d'entrevues dans lesquels ils nous présentent différents aspects pratiques de leurs tâches. Notamment, leurs réflexions au sujet de leurs actions et leurs explications relatives aux stratégies qu'ils adoptent ou qu'ils préconisent afin de faire face aux défis rencontrés.

\section{IV.4.a - Ce que fait l'expert}

Selon les personnes interrogées, l'expert procède à des activités qu'il répète. Dans le cadre d'une ou de plusieurs de ses activités, l'expert identifie différentes situations d'expertise, des difficultés et des tensions à propos de la nature des processus de développement. En ce sens, l'expert est constamment appelé à consulter et invoquer des savoirs tirés des rencontres, des conférences et des publications du monde académique dans le but de poursuivre l'une ou plusieurs de ses activités. Dans le cadre de son travail, avec l'appui de ses expériences, l'expert fait de manière routinière, des recommandations, des évaluations et des suivis, conçoit des projets, enquête sur des projets ou encore transfére ses connaissances. Dans la section suivante, les experts abordent chacune des actions énumérées.

\section{Faire une recommandation}

L'activité la plus connue et la plus commune chez l'expert est la recommandation. Celle-ci suit un processus analogue d'un projet à l'autre, en effet il fera un lien entre la situation qu'il observe et la 
littérature scientifique. Pour Frank, fonctionnaire et économiste de l'environnement, cela signifie que :

Quand tu fais une recommandation, tu vas voir s'il y a un consensus dans la littérature, s'il y a plein d'académiciens, de «think tank» qui convergent dans leur opinion, s'il y a une théorie prépondérante là-dessus; c'est relativement simple quand il y a un consensus scientifique. C'est beaucoup moins évident quand il n'y a pas de consensus au niveau académique.

L'utilité du processus de recommandation réside dans le fait que cette dernière est suggestive. Elle devient ainsi utile ou malléable quand elle convient au demandeur, suscite la réflexion ou encore, devient « jetable » quand elle ne convient pas au demandeur. Frank explique que:

Les recommandations d'experts ne vont pas nécessairement être retenues, ça peut influencer le processus décisionnel, comme ça peut être complètement ignoré. Tes clients ou les élus peuvent faire exactement le contraire que ce que tu recommandes. ... Des recommandations non demandées peuvent aussi faire allumer des lumières et susciter des commentaires positifs et provoquer un changement de fusil d'épaule.

Le processus d'expertise répond ainsi à un besoin de justification et quand il ne répond pas aux besoins du demandeur, la tâche de l'expert est d'accepter ce destin selon Carl : « les recommandations, des fois les organismes les ignorent et c'est très bien. L'avantage de faire appel à un consultant c'est qu'on est pas obligé de suivre son avis... pour certains experts, il faut que l'égo soit capable de le prendre! » Si ignorer une recommandation peut être perçu négativement par certains experts, c'est qu'elle est souvent le moment intense du processus d'évaluation et de suivi d'un projet comme l'explique Marie :

En général une recommandation ne tombe pas du ciel comme ça sans qu'on s'y attende. En général, je parlais déjà au monde d'amélioration positive que l'on pouvait faire. Donc, je parlais avec les gens, je travaillais avec les gens, le rapport c'est à moi, ce n'est pas fait avec les gens de la base, mais évidemment, on discutait avant et puis parfois ils pouvaient m'éclaircir me dire tu as raison pour ça, mais on fait ça, à cause de ça; ils me donnaient des éléments que je ne connaissais pas. Dans mon cas personnel, les conclusions du rapport étaient discutées avant, et ils savaient ce qui allait s'y trouver. 
Selon les personnes interrogées, la recommandation d'un expert intervient donc dans un cadre précis et fait partie du dialogue qui a cours lors d'un processus d'évaluation et de suivi.

\section{Évaluations et suivis}

Les évaluations et les suivis qui mènent aux recommandations sont quant à eux des activités plus complexes. Lorsque la demande d'expertise vient d'un organisme qui finance un projet, la plupart du temps, l'expert se trouve en situation d'évaluation du projet à partir de normes identifiées et cadrées par l'organisme demandeur. Comme c'est le cas à l'ACDI, ce type d'évaluation normative ne laisse pas de place pour la discussion. La personne interrogée Maurice en explique la réalité à travers cette envolée critique des cadres d'évaluations de l'ACDI :

[l'ACDI] te donne les résultats à court, moyen et long terme et [l'ACDI] disent ce qu'ils vont utiliser pour faire ça. T'appelles ça le cadre logique ou le modèle logique, et tu regardes les attentes qui sont atteintes. Ça t'amène pas souvent à t'ouvrir sur une grande discussion, mais t'en arrives à faire des observations $[\ldots]$ Certaines sont pas toujours exprimables parce que t'as beau leur dire, vous visez trop grand; ils vont te dire : « ça c'est pas de tes affaires, c'est ça qui est prévu, analyse [ce qu'on t'a demandé] on a dit qu'on travaille avec 10000 personnes; est-ce qu'on travaille avec 10000 personnes? » Oui. Alors je les reprends sur les résultats; les résultats sont minimes. S'il y avait eu moins de personnes, ça aurait été [plus significatif]. "On veut pas savoir ça, on veut savoir... " tu vois l'idée... Il reste que toutes les organisations, et puis c'est normal, tu veux avoir des résultats qui touchent un grand nombre de personnes, mais c'est pas réaliste selon moi avec les budgets qu'on ait d'avoir autant d'attentes face au peu d'argent qui est mis là-dedans. Et puis c'est pareil pour tous les pays.

On considère que le temps que l'expert passe sur le terrain lui confère une connaissance assez étendue des normes de l'organisme demandeur, de son rôle d'expert et des situations sur le terrain. Ceci lui permet de traduire les événements du terrain en critères d'évaluation. Maurice explique :

On me dit souvent, oui, mais toi qu'est-ce que tu connais en santé? J'ai pas besoin de savoir comment opérer, j'ai besoin de savoir s'il l'a fait. C'est marqué dans les documents qu'il a fait 20 opérations alors qu'il me dit qu'il en a fait huit. «As-tu des problèmes au niveau techniques? Des 
problèmes avec le personnel? Oui, non, oui, non, explique-moi donc maintenant, l'entrevue est un petit peu plus ouverte, on va parler en général de la gestion de l'hôpital puis là tout sort. Tu les amènes à te parler puis à te confier [les problèmes qu'ils ont]. Les problèmes qu'ils ont, ils les savent, c'est de les amener à le dire [le défi] beaucoup plus que de les régler, ils le savent puis là ils connaissent ça bien mieux que toi.

On peut placer l'évaluation que font les experts des processus de suivis de projet sur un continuum. Dans ce continuum, on observera d'un côté que certains effectuent l'évaluation dans un esprit d'ouverture ou d'implication sur le terrain par rapport au projet concerné. À l'opposé, certains suivis et évaluations sont purement protocolaires. Audrey partage son appréciation d'un genre de processus qu'elle qualifie d'ouvert :

Dans des dynamiques d'évaluations, les chargés de projet on est souvent extrêmement positifs. Il existe principalement deux types de rencontres qui vont servir à faire des suivis et des évaluations. Il y a d'abord le suivi et l'évaluation de travail : dans lequel des évaluateurs externes viennent faire un suivi du projet, des problématiques rencontrées des solutions utilisées, pour voir comment on peut mieux investir l'argent. C'est pour nous extrêmement motivant, car il y a des échanges et des discussions, souvent un regard neuf qui nous aide à nous améliorer.

Selon Audrey, coopérante et spécialiste de la communication sociale, la venue d'un évaluateur externe est tout le contraire des visites protocolaires qui consistent essentiellement à « se gargariser de nos succès. » Gérald, coopérant et spécialiste de la question du genre, abonde dans le même sens : la visite protocolaire revêt souvent une forme à la limite du « burlesque ». « Les gens de l'ONG arrivent pour voir où l'argent est allé. Ils vont arriver là en quatre-quatre, ils ont un petit show musical pour eux, ils ont fait des tatas, ils parlent au chef du village, puis ils s'en vont. » Une situation qui pour Audrey est problématique : «Ce genre de rencontre est biaisée, car elle tait les problèmes des projets et les difficultés que nous n'arrivons pas à surmonter. » 


\section{Enseigner l'ingénierie inverse de l'évaluation}

Dans d'autres contextes, l'expert travaillant directement avec les communautés transfèrera une partie des connaissances acquises dans son travail d'expert en développement, dans un cadre où il prend position et se retrouve dans le rôle de l'évalué. Pour certains, ce sera l'occasion d'expliquer les attitudes, les coutumes et les «qualités » à démontrer pour obtenir les faveurs de ceux qui distribuent les ressources des agences et des organismes. Carl nous explique : « Quand les organisations évaluées sont en mode survie, elles se démènent pour s'assurer que l'on voit surtout leurs bons côtés. » Pour un organisme placé dans une telle situation, le centre de sa préoccupation est la perception du bailleur de fonds. On use alors de stratégies pour « fabriquer » une légitimité et maintenir un projet en vie, comme l'évoque Gérald :

On crée souvent des mises en scène pour plaire aux bailleurs où on va s'arranger pour faire un travail public, par exemple donner une formation au moment où on vient nous évaluer alors que cela représente une infime partie du projet, mais c'est celle qui en apparence permet de maintenir son image de bon travailleur ou de bon organisme de développement.

Cela donne un aperçu des processus d'évaluation aux gens sur place, qui utilisent ensuite ces stratégies pour maintenir leur financement. Bien au courant que des subterfuges ont lieu, l'expert qui évalue, suit les progrès, recommande ou transfère des connaissances doit alors chercher à comprendre ce qui se passe « réellement» derrière la mise en scène évoquée précédemment.

\section{Enquêter pour détecter la mise en scène du développement}

Ainsi, un expert responsable de l'évaluation effectue des vérifications au regard d'un cadre normatif dans une temporalité restreinte. Simultanément, il détecte si la documentation produite selon 
les critères attendus par l'évaluateur est arrangée ou vérace. . Maurice, anthropologue et évaluateur pigiste, explique le travail d'enquêteur de l'expert :

Je suis arrivé sur un projet d'environnement très, très, très, intéressant. Ils devaient faire des filtreurs en ciment, c'était une nouvelle technologie, c'était novateur t'avais besoin la première des choses de coulées du ciment en entonnoir, moi j'arrive là puis c'est pas fait. Je vois un tas de gravelle pour mixer le ciment, et des poches de ciments un peu plus loin. J'arrive là, la gravelle est foncée : ça veut dire qu'elle est mouillée encore, qu'il vient de l'amener là ce matin. Il y a les poches de ciment pour couler le quart de l'entonnoir qui est là. Et bien ça c'est quelque chose que je sais avec le temps, je leur dis : bien là les gars, vous êtes venus porter de la gravelle à matin, elle est mouillée... Vous avez tant de poches de ciment: vous faites pas un entonnoir de ciment avec ça là... On va reprendre ça du début : où est-ce qu'on est rendu ici? » Là ils se disent « Ah lui on le pognera pas... » Tu fais ça gentiment-là, tu les attaques pas, il y en a qui vont les attaquer, chacun son approche, moi j'ai une approche qui est plus « je vais vous expliquer ce que je vois » et habituellement c'est assez pour les amener à avoir une discussion pour dire : «OK OK on a des problèmes, on s'est fait volé les outils », admettons ou " écoute ici c'est très dure d'avoir du ciment, car il y a une priorité dans telle province et tout le ciment est allé là. On s'est pas assez battu, c'est pas prioritaire, puis la vérité c'est que ce sera pas fait avant six mois. » Il faut tu ailles chercher ces informations-là. C'est pas une expertise à tout casser, mais ce que tu veux c'est produire des résultats tel que prévu.

Ce travail d'enquête exige des ressources importantes afin de pouvoir établir un portrait fidèle et à jour de la réalité selon les normes évaluées. Or, pour Frank, il y a un manque à ce niveau qui mine le travail de l'évaluateur et le processus au complet : «Si on a les ressources nécessaires, on peut faire une évaluation adéquate des programmes et des projets. Or c'est rarement le cas. » Ces évaluations servent ensuite à concevoir, à gérer et à améliorer les projets.

\section{Concevoir, mettre en place, gérer ou promouvoir des projets}

Si certains font plus souvent de l'évaluation, d'autres experts se servent des expériences accumulées dans les projets précédents pour mettre en place des projets pré-montés sur demande. Ils doivent employer le modèle et le cadre de développement choisi, faire les liens avec les partenaires choisis, et le cas échéant, promouvoir la réussite du projet. Ils créent ainsi un produit de développement destiné à être « acheté » par le bénéficiaire tel que l'évoque Maurice : 
J'ai mis en place des modèles de développement commandé au PNUD par un pays bénéficiaire ou à d'autres agences de ce genre. Tu sais, c'est de même que ça marche, le PNUD arrive ils te disent « on a un bon modèle... ... ... ça vous intéresse? » eux ils appellent ça... la coopération décentralisée. Le PNUD, ils vont aussi fournir des partenaires qui vont travailler avec les bénéficiaires. En même temps, tu te retrouves à avoir une base de financement décentralisé, ça intéressait les agences bilatérales parce qu'ils se disaient, « oui, ça fait beaucoup de partenaires, puis on peut travailler en synergie, en harmonisation...» tous les termes, les thématiques à la mode peuvent être ainsi invoqués. Ensuite quand ça réussit, tu fais un petit pamphlet là-dessus pour faire la diffusion des résultats d'innovations prometteuses dans tous les pays du sud pour les intéresser à ce type de projet. Mon rôle était de créer un cadre stratégique pour vendre l'idée, expliquer l'idée, rencontrer les donateurs, expliqués aux donateurs, puis faire un cadre pour dire que c'est de même que ça va fonctionner.

Pour pouvoir faire cheminer l'ensemble de ces productions, évaluations, suivis ou devis de projet, d'autres tâches connexes doivent être accomplies.

\section{Effectuer des tâches bureaucratiques}

Si les tâches principales de l'expert consistent en l'évaluation, le suivi et la conception de projets, voire l'enseignement, celles-ci s'inscrivent dans un cadre bureaucratique. L'expert est donc appelé à accomplir nombre de tâches bureaucratiques en lien avec ses tâches principales. Ces tâches servent principalement à rendre compte des suivis, des évaluations, des conceptions et de la mise en place de projet. Selon Gérald, «[l'expert] a des échéanciers à respecter, il doit respecter des critères d'évaluations, il a des documents à remplir, il doit faire des révisions des documents produits, etc. \" pour pouvoir obtenir des fonds nécessaires aux fonctionnements des projets. Par contre, tous les experts n'ont pas à effectuer le même niveau de tâches bureaucratiques. Selon la stratégie de travail choisie ${ }^{15}$, comme l'explique Leonard :

Il faut faire des propositions, des budgets et de la planification qui lorsque fait par une seule personne peut être très prenant sur autre chose que l'évaluation elle-même. Les firmes ont un avantage sur ce plan dans le sens où elles sont plus efficientes dans le polissage des rapports

15 Nous examinerons plus en détail les stratégies d'insertion dans le marché du travail plus loin dans le présent chapitre p.102 
(bibliographie, présentation qualité de la langue, annexe), la gestion des finances et le remplissage de propositions, la planification des voyages, etc.

On peut donc présumer que dans certains cas, le temps devient un capital très valorisé par les experts. Plus un expert est en mesure d'allouer du temps à du travail d'expertise et de déléguer les tâches bureaucratiques à d'autres, plus il est capable de se concentrer sur des activités d'expertise.

Dans le cadre de son travail, avec l'appui de ses expériences, l'expert est appelé à faire de manière routinière des évaluations, des suivis, des recommandations, à concevoir des projets, à enquêter sur des projets ou encore à transférer ses connaissances. Ainsi par exemple, selon la tâche qui lui est assignée, un consultant pigiste indépendant pourrait faire un rapport d'évaluation du volet environnemental d'un projet spécifique avec des recommandations. Un coopérant volontaire quant à lui évaluera des projets d'un partenaire d'une ONG et en profitera pour transmettre quelques trucs aux gens sur place pour paraître à son avantage face à l'évaluateur du gouvernement canadien, alors qu'un consultant travaillant pour une firme d'expert en développement évaluera les projets financés par un programme de l'ACDI au Ghana afin de déterminer si le projet se déroule comme prévu. La liste des possibilités observables devient longue. Devant cet éventail de situations, il importe de savoir ce qui amène les experts à réfléchir dans l'objectif de comprendre leur perception du monde et sa catégorisation afin de bien saisir le contexte dans lequel ces situations ont lieu.

\section{IV.5 - L'expert réflexif}

L'expert doit porter plusieurs chapeaux, soit celui d'expert et d'expatrié. À cet égard, dans plusieurs situations, l'expert est réflexif. En tant qu'expatrié, ses réflexions sur sa forme d'intégration aux communautés assignées et sa manière de vivre en Afrique nous informent sur les différentes 
stratégies utilisées pour transmettre ses idées. D'autre part, elles nous renseignent sur des situations que l'expert ne souhaite vivre et à l'opposé sur celles qu'il recherche. Dans cette section, nous allons aborder les réflexions des experts relatives à leur conception de la notion de conflit d'intérêts, de leurs motivations à œuvrer en développement international, de leur manière de s'intégrer dans les lieux d'assignation et des situations qu'ils souhaitent éviter.

\section{Le conflit d'intérêts}

Tout d'abord, l'expert qui réfléchit sur l'éthique nous permet d'identifier ce qu'il estime nécessaire pour ne pas entrer en conflit d'intérêts. En développement, un spécialiste de l'évaluation précisera qu'il ne proposera jamais ses services pour faire une recommandation sur la manière de faire les choses et par la suite ses services pour mettre en œuvre ses propres recommandations. Frank est catégorique à ce sujet : « Je ne rentrerai jamais dans un endroit pour leur dire comment faire et faire de la mise en œuvre. » Le conflit d'intérêts constitue la situation à fuir et à éviter. Ainsi, pour plusieurs, il existe un malaise face à des entreprises, par exemple une firme d'ingénierie ou un cabinet comptable qui parmi leurs activités proposent des expertises en évaluation de projet et en mise en œuvre. Bien que plusieurs affirment ne jamais adopter de tels comportements, le fait qu'ils existent provoque un malaise face aux personnes pratiquant l'expertise en développement dans de telles situations. Ainsi, le comportement qui trace la limite entre le conflit d'intérêts et la relation acceptable de l'expert serait marqué par le fait de se trouver à évaluer son propre travail selon Frank

... t'as aussi des entreprises qui vont faire un peu des deux, ils vont faire de l'analyse et de la mise en œuvre de programme sans nécessairement faire des briques et du ciment. Ils vont aller sur le terrain et aider le ministère des Finances pour établir un bon budget national, ils vont faire les deux, l'évaluation et la mise en œuvre. Ce qui se trouve à être un peu touchy quand tu fais de l'évaluation de programme, c'est que tu te trouves à être en conflit d'intérêts si tu es appelé à évaluer un programme que tu as géré, que tu as mis en œuvre [...] je sais de façon anecdotique que ça arrive, je n'étais pas impliqué. L'entreprise a dû être mise de côté parce qu'elle n'avait pas un bon plan de 
gestion pour mettre de côté et gérer ce risque-là, de perception de conflit d'intérêt et de conflit d'intérêt réel.

En ce sens on comprend donc qu'accomplir l'évaluation avant de faire la mise en œuvre de ses propres recommandations est vue comme moins problématique sur le plan éthique que d'évaluer son propre travail.

\section{Le choix de la communauté d'appartenance}

Pour d'autres, la notion de conflit d'intérêts est plus large et se manifeste dans les choix de vie de tous les jours, notamment à travers le choix de la communauté d'appartenance et les motivations derrière l'intérêt porté au développement. Par exemple, en raison de leur traitement financier et de la courte nature de leur assignation, certains experts dépêchés sur le terrain passent la plupart de leur temps libre en ville ou dans les capitales, dans les hôtels et restaurants, souvent sans leurs familles, proches ou amis, dans ce que Leonard qualifie de « reconstitution du mode de vie occidental $»^{16}$. Marie nous décrit ainsi une première forme de cet engagement, celle de l'expertise en retrait du milieu assigné, en faveur d'un repli dans le mode de vie occidental.

Les consultants classiques se retrouvent souvent au même endroit sur le terrain, coupés des populations dans les mêmes établissements, restaurants, bars, hôtels. Surtout en Afrique, ces comportements sont encouragés par les ambassades qui y organisent fêtes, banquets, réunions et soirées de rencontre. C'est une structure entraînante par le luxe qu'elle affiche.

Un point de vue partagé par Gérald pour qui

... il y a les lieux informels de détente, les centres culturels français, les piscines, les hôtels, un bon moyen pour trouver un coopérant, c'est d'aller à la piscine. Puis il y a les restaurants haut de gamme où les repas coûtent entre 5-10 $\$$ par personne où il y a des locaux, mais principalement des experts et des coopérants étrangers.

16 Notre traduction: " reenactment of the western way of life. " 
En d'autres termes, les endroits que fréquentent majoritairement les experts sont des lieux où le train de vie exclut la majorité des populations locales de même que les populations ciblées par les projets.

Pour Leonard, cette forme d'engagement a un effet tant sur le plan individuel que psychosocial, celui de l'exclusion de la vie sociale « normale » pour une vie de voyageur sans point d'ancrage, sans référence :

Les experts passent beaucoup de temps entre eux, à l'hôtel, à socialiser avec d'autres voyageurs. Vivre dans les hôtels ne permet pas de s'enraciner dans une communauté, parce que votre maison est dans vos bagages. Cela implique un coût important pour ceux qui ont des familles par exemple, maintenir une liaison à long terme devient difficile quand on est parti. Il est difficile d'entretenir les amitiés, vous ne faites pas partie de la scène sociale: vous êtes indisponible. Cet état de fait pourrait contribuer à une certaine forme d'aliénation sociale, une certaine forme de stress. La plupart des gens que je connais qui ont eu ce style de vie reconnaissent qu'elle a un coût au niveau personnel.

Pour certains, une telle stratégie d'engagement dans les communautés ciblées se révèle douteuse sur le plan éthique. Pour eux, ce type d'engagement entraine une séparation qui nuit à la communication destinée à susciter le développement. Cette séparation est créée en pratiquant des activités qui différencient « l'élite » dont l'expert fait partie, de la communauté locale. Ainsi pour Marie, «On fabrique une super structure élitisante du développement, par exemple, si un consultant soumissionne trop bas pour une agence, on ne prendra pas sa candidature parce qu'on a l'impression qu'il est un amateur, « un gars du bac », bref qu'il ne fait pas partie de cette élite, du monde du développement. » Dans la réalité, les experts ont tendance à se catégoriser entre eux sur cette question et proposent des pôles bien définis, mais tout en acquiesçant qu'il existe une mobilité entre ces deux pôles comme le fait Gérald :

... il y a deux types de réflexes chez les experts, chez les coopérants, nomme-les ce que tu veux, il y 
a ceux qui vont aller plus vers l'autre blanc, l'autre étranger, lui demander d'où il vient, dans quoi il travaille, qu'est-ce qu'il fait ici. Il y en a d'autres qui ne veulent rien savoir : ils vont aller vers les autochtones, sauf qu'eux tu les vois moins parce qu'ils se tiennent pas dans les endroits «blancs», ils se tiennent dans leurs quartiers alors que quand tu vois une fille blanche dans la rue qui s'en va au restaurant, tu as une image qui est plus prévisible... Ces comportements-là ne sont pas figés, ils peuvent s'améliorer si on veut. Ces comportements peuvent être transcendés, ça dépend de la volonté des gens. Il y a beaucoup de gens qui aime ça vivre leurs petits trucs de manière fermée, autant il y en a qui en sont incapables, autant il y a des gens qui vivent entre les deux. Autant je suis quelqu'un qui n'aime pas les petits ghettos, autant ça m'est arrivé d'y aller. Ça n'est pas des rôles fixes, il y a une transcendance entre les deux, ce sont deux pôles et là-dedans, il y a des va-et-vient, c'est sûr qu'il va y en avoir qui se tiennent plus près d'un pôle, mais il y a beaucoup de va-et-vient.

Invariablement, les critiques (Audrey, Gérald, Marie) de ceux qui vivent en marge des communautés axent ces dernières sur les motivations en lien avec les choix d'œuvrer en développement et d'exercer le rôle d'expert.

\section{La motivation à ouvrer en développement}

Cette critique du choix du lieu et la communauté de résidence de l'expert alors qu'il poursuit des activités de développement en Afrique subsaharienne ouvrent la porte à une réflexion à propos de ces multiples motivations. Elles s'étendent sur un continuum où l'on trouve des motivations humanistes et revendicatrices à un extrême et des motivations d'ordre carriériste et pécuniaire à l'autre. C'est ainsi que les personnes rencontrées font appel aux valeurs humanistes pour motiver leur engagement vis-à-vis du développement en faisant référence au goût d'apprendre, à la curiosité de l'autre, à l'amour des gens, à l'atteinte de leurs objectifs et à la transformation positive, entre autres. Pour Marie,

... la motivation, c'était de connaitre la façon de vivre et de pouvoir acquérir une expérience humaine. J'ai pu contribuer du point de vue professionnel, mais du point de vue humain, je me suis enrichie beaucoup, beaucoup, beaucoup, et ça, ça ne se mesure pas. Concrètement, ça m'a apporté des amitiés pour toute la vie par exemple, de connaitre d'autres cultures différemment d'un touriste.

Gérald s'inscrit dans un même registre, car «pouvoir percevoir une transformation positive vers 
des meilleures conditions de vie ou un signe de celle-ci chez les communautés où il œuvre ») constitue sa motivation principale. Or, poursuit-il, « en principe [...] un expert devrait travailler pour les populations précises visées par le projet. » On comprendra ici que certains experts ont une action plus orientée vers la poursuite de leur carrière personnelle, voire ont un intérêt pécuniaire influençant les moyens choisis pour faire des recommandations. L'expert se trouve ainsi dans un constant dilemme éthique entre le maintien de son statut social et économique, le maintien d'une proximité avec les populations nécessaires à la compréhension des problématiques visées par son travail d'expert. Un dilemme qui se traduit dans ses choix.

D'autres personnes rencontrées élargissent l'horizon du recours à la motivation pour l'appliquer à la critique des routines de l'expertise canadienne destinée à l'évaluation de projet, plus précisément à ce qu'elles font et ce qu'elles ne font pas. Par exemple, Audrey sous-entend que les motivations derrières l'évaluation ne se font pas sur une base égalitaire ce qui lui permet de remettre en question l'unilatéralité de l'évaluation, c'est-à-dire l'évaluation par ou pour les donateurs sans processus semblable pour le récipiendaire, car « [i]l existe une incohérence dans le monde de l'évaluation, les partenaires du sud ne viennent jamais évaluer ce qui se fait dans le nord. Ce n'est pas franc ni honnête dans un cadre qui se veut de collaboration et de coopération. Les protocoles n'exigent pas ce type de retour. » Audrey ne s'arrête pas seulement à la critique de l'évaluation, mais remet en cause tout le discours sur l'aide au développement à partir de l'étymologie de mots et de proverbes locaux ce qui démontre son rapprochement avec les intérêts locaux où elle explique : «le terme "aide" au développement me rend mal à l'aise, car il implique une relation de supériorité. Il existe un proverbe malien qui résume bien ma pensée à ce sujet : "La main de celui qui donne est toujours au-dessus de celle qui reçoit". » 
Pour d'autres, il est difficile de s'affranchir de l'institution pour laquelle on travaille ainsi que de ses motivations. C'est particulièrement le cas pour les experts qui travaillent pour une firme et qui doivent rendre cette dernière profitable comme l'expose Carl : «Les firmes de consultants sont des entités d'affaires; elles vont se nourrir de toute forme de travail qu'elles vont trouver. Dans ce cas-ci, avoir une philosophie du développement n'a aucune importance. »

Dans une relation où, à la base, on accorde à l'expert une forme de supériorité à travers l'autorité qui lui est conférée par ses mandataires, la principale difficulté à faire « une bonne expertise » pour Marie est de maintenir les bases d'une communication égalitaire pour agir avec les communautés visées vers une action de développement appuyée par l'examen des connaissances de tous; experts et expertisés :

Le défi surtout en Afrique c'est le fait de faire attention à ne pas se mettre au-dessus des gens. Nous on arrive d'occident en croyant avoir la vérité, en critiquant certains modèles culturels. Le grand défi c'est d'être d'abord à l'écoute, après penser, analyser, et troisièmement donner son opinion pour faire avancer. [...] Le respect de l'autre culture c'est d'écouter, d'entendre, de voir avant de s'exprimer et d'apporter nos solutions toutes faites.

Pour Frank, les problèmes techniques du développement sont essentiellement dus à un manque de capacité d'analyse, car « [c]'est pas l'information qui manque, ce qui manque vraiment c'est la capacité de résumer cette information et d'apporter des recommandations par rapport à ce qui est l'information de pointe dans le fond. "

Gérald pousse l'idée plus loin et estime que chez les experts, le manque d'expérience de situations vécues par les populations les plus vulnérables explique leur manque de capacité d'analyse, voire de compréhension, des dynamiques du terrain : «Si tous les experts avaient vécu là-bas avec une "piasse" 
par jour, là ils pourraient peut-être mieux comprendre. L'idéal c'est d'être près du terrain pour comprendre ses logiques et comment on peut intervenir dans ces logiques. »

Ces remises en question poussent Carl à faire un retour sur les avantages et les désavantages de l'organisation du partage des savoirs et des connaissances en développement à travers des mécanismes auxquels l'expertise a recours :

L'expertise est source de tension parce qu'elle n'est pas toujours utilisée pertinemment. Il existe une chose telle que l'expertise, car des gens accumulent du savoir et de la connaissance dans certains domaines et cela s'avère fréquemment utile. Dans d'autres les experts sont de bons vendeurs, ils sont très bons pour convaincre que l'on a besoin d'eux sans que ce soit nécessairement le cas.

Les motivations notamment revendicatrices nous apprennent que le recours à l'expertise joue un rôle clé dans la mise en œuvre d'un type de développement plutôt qu'un autre.

\section{Les histoires d'horreurs}

Ce rôle c'est celui de contrôler le risque associé à la mise en œuvre de projet de développement, car «l'Histoire est jalonnée de développements inappropriés » nous rappelle Carl. À cet effet, les

experts émettent des critiques sur le développement. À titre d'exemple, la critique du développement par ses « histoires d'horreurs » implique à tout coup, de près ou de loin, des experts et de l'expertise. Il se développe autour de ces histoires d'horreurs des mythes qui rappellent le risque que l'expert doit contrôler, c'est-à-dire au sens où Barthes l'entend que ces histoires deviennent une clé d'interprétation de la réalité qui est moussée par le groupe qui les véhicule (Barthes 1957). À partir de ce qu'on peut retenir des propos des personnes interrogées, on peut diviser ces mythes basés à la fois sur de réelles histoires de développement et sur l'idéologie promue par le développement en cinq genres distincts; 
soit, « l'éléphant blanc », la « fausse bonne idée », la «récupération du développement au profit des entreprises», la « sous-traitance mal avisée » et la « course à la qualification ». Les prochaines sections présenteront les cinq mythes distincts dans leur version telle qu'évoquée par les personnes rencontrées.

Parmi les histoires d'horreur, « l'éléphant blanc » correspond à un projet pour lequel il y a eu un financement et laissé à l'abandon. Le mythe de « l'éléphant blanc » est à la fois ce qui justifie la présence de l'expert parce qu'il est là pour prévenir sa matérialisation, et le résultat qui demeure le plus redouté de tous les experts, car il constitue la preuve matérielle de l'échec de l'expertise ce qui provoque la critique de l'expertise et de sa légitimité. Ainsi c'est une histoire récurrente qui est toujours présente dans les discussions: Frank nous indique qu'« [aujourd'hui] ce sont des situations plus anecdotiques que générales, je ne pense pas qu'il y ait eu des évaluations vraiment holistiques par rapport à ça, mais on en entend de façon anecdotique des histoires par rapport à des projets qui donnent des «l'éléphant blanc». D'autres sont plus directs et affirment avoir été les témoins directs d'un « l'éléphant blanc». Marie expose cette anecdote ainsi «j'ai eu à évaluer un projet d'une ONG bien connue et lorsque je suis arrivée, il n'y avait tout simplement pas de projet. Malgré mon signalement de la situation à mes supérieurs, l'ACDI et l'organisme canadien responsable du projet ont tout de même continué à financer l'organisme local en raison de son statut et de la reconnaissance qu'il avait... » Ainsi, l'éléphant blanc peut soit avoir une trace physique telle qu'une construction inutilisée ou soit, constituer en une absence totale de projet. À l'opposé, d'autres projets existent réellement, mais dans les faits profitent peu à la communauté ciblée.

Pour les experts, le développement est jalonné d'idées qui sont bonnes sur papier et qui atteignent de bons résultats dans les paramètres d'évaluation, mais qui dans les faits aboutissent à un résultat qui dégradent le niveau de vie des populations locales. La « fausse bonne idée » consiste à utiliser une 
technologie inappropriée. À ce titre, Carl évoque rapidement le cas des « fameuses cultures de blé tanzanien financées par le Canada $»^{17}$ lorsqu'on lui demande si l'expert peut nuire au développement. Avec un transfert de technologie pour trame de fond, ces histoires ont pour point commun l'utilisation d'une technologie inadéquate par rapport au contexte économique, social et géographique dans lequel la technologie est transférée, ce qui a pour résultat de laisser en plan les populations locales, voire de détruire leur mode de vie. Dans des cas comme ceux-ci, l'horreur se manifeste dans l'erreur de jugement ou dans cette variable oubliée qui affecte dramatiquement la vie des populations visées. Or, dans d'autres cas, on ne parle plus d'une simple erreur, mais de situation où le projet est organisé sciemment dans le but d'orchestrer une forme de bénéfice qui revient au bailleur de fonds.

Dans une moindre mesure, mais dans une proportion tout de même importante, « la récupération du développement au profit des entreprises » qui financent des projets constitue un second événement redouté par les experts rencontrés. Selon Frank, :

Un exemple de ça c'est la nouvelle tendance à l'ACDI [...] où ils ont un gros focus sur le partenariat avec le privé en développement international. Il y a plusieurs années, l'ACDI avait une boîte qui s'appelait CIDA inc. C'était un partenariat avec le privé pour faire des projets de développement international. C'était bien avant mon temps, mais tu te trouves à avoir des incitatifs qui sont bien différents. Les incitatifs de l'ACDI, c'est que leur projet de développement se traduise en création d'emploi et en amélioration du sort de ses citoyens. Tandis que l'industrie privée est de générer des profits qui se trouvent dans certains cas à concorder avec les intérêts du pays récipiendaire ou celui du Canada, mais dans la plupart des cas, ça concorde pas. Tu peux imaginer un paquet de scénarios où le profit; la décision qui est la plus profitable va pas nécessairement concorder avec la décision qui est la plus avantageuse pour la population locale... En termes d'impact sur la population locale, c'est clair que si c'est un projet qui est géré plus par rapport au profit que par rapport à la communauté, les gens vont être moins engagés, ça va être beaucoup moins durable, une fois que l'entreprise est partie.

L'objet de l'horreur est une privatisation du développement. Dans un tel cas, le développement

17 Tanzania Canada Wheat Programme (TCWP) un programme actif de 1970 à 1993, bien qu'évalué positivement par l'ACDI et le gouvernement tanzanien, ce programme a eu un impact dévastateur sur le peuple Barabaig (Lane et Pretty 1990; Lane 1994; Stackhouse 2001). 
sert à faire de la subvention publique une recherche de profits et de rehaussement de l'image des entreprises canadiennes dans des pays en développement, ce qui s'oppose parfois à l'objectif d'améliorer les conditions de vie dans les communautés visées. Or, si de tels cas sont facilement identifiables, voire médiatisés (Leblanc 2012; Oved 2014), d'autres utilisent plutôt les contrats de soustraitance de l'évaluation des projets pour retirer un profit au détriment de la qualité de l'évaluation des projets.

$\mathrm{Si}$, pour le cas de la récupération du développement, le lien est plus évident entre les entreprises et les situations problématiques, les firmes d'experts peuvent avoir des comportements internes qui laissent planer un doute sur la qualité de l'expertise mise à contribution. L'évaluation du rendement de la coopération, de la solidarité et du développement fait souvent l'objet d'une sous-traitance. Les dérives dans « la sous-traitance mal avisée » font partie du lot de ce qui se passe à l'intérieur des firmes. «Quand tu travailles comme consultant [pour une firme], il y a une pression à se concentrer à facturer le plus possible le client et à trouver des consultants plus jeunes avec moins d'expérience qui feront le travail pour moins cher», mentionne Leonard. Le milieu de la pratique privée de l'expertise en développement rend ainsi propices les dérives, telles que la pression à la facturation, la figuration des véritables experts, les évaluations de moins bonne qualité faites par des gens avec moins d'expérience dans le but de défrayer les coûts de sa bureaucratie interne et de dégager un profit pour l'entreprise.

La compétition pour les emplois est rude dans le domaine privé et le succès rapporte monétairement à ceux qui s'y distinguent. Or, ce ne sont pas seulement les acteurs liés directement au développement international qui profitent de ce climat, car comme on l'a vu plus tôt, les qualifications académiques sont très prisées dans le monde du développement. De cette façon, les institutions d'enseignement profitent de la pression qu'engendrent les compétitions internes dans les entreprises et 
alimentent de diverses formations « la course à la qualification ». Ces diplômes y sont perçus et promus comme un atout pour augmenter ses chances d'obtenir un emploi bien rémunéré et plus stable. Selon Audrey, « il y a une récupération de la coopération internationale à travers la création de « diplômes professionnalisants » qui en porte l'appellation. » À la lumière de ces informations, nous pouvons dresser l'image d'un monde où l'université marchande vend un éventail de produits professionnalisant la coopération et la solidarité; diplômes, certificats, maîtrises et doctorats sont ainsi offerts aux étudiants et travailleurs du développement qui souhaitent devenir experts et améliorer leurs chances d'avoir accès aux postes favorisant le développement de sa carrière et la sécurisation de sa place dans le domaine. En ce sens, le monde académique profiterait du besoin qu'ont les travailleurs de justifier leur expertise dans un secteur de plus en plus compétitif pour des ressources qui se font de plus en plus rares. Or, cette preuve de qualification est demandée par le cadre gestionnaire qui cherche à avoir le plus de compétences possibles pour un prix minimal.

\section{La pression des impératifs gestionnaires}

Ainsi pour un expert déjà établi, comme Maurice, le cadre gestionnaire et la pression qu'il impose sont sources de plusieurs comportements, comme travailler des heures supplémentaires bénévolement, adapter les résultats de son travail aux objectifs d'un projet ou encore censurer les conclusions qui iraient à l'encontre d'intérêts politiques. À titre d'exemple, Maurice souligne : « Il y en a même qui vont changer les chiffres [dans leurs rapports]... ... Ça arrive ça là, pas tout le temps, mais disons qu'il y a ce risque-là parce qu'il y a un manque de financement. " Selon Frank cette situation est imputable à la nécessité pour l'expert de ramener des contrats.

C'est beaucoup plus "touchy" comme consultant parce que tu te trouves à avoir un incitatif qui est différent $[\ldots]$ t'es guidé par ton intégrité et ta réputation à long terme comme consultant et entre le 
deux t'as le profit à court terme. Si tu agis en pensant seulement qu'au profit à court terme, évidemment tu vas rédiger des recommandations qui sont exactement ce que le client veut entendre, mais à long terme tu te trouves à miner la crédibilité de ton entreprise. Donc t'as du profit à court terme parce que t'as plu à ton client, mais tu peux établir une réputation comme étant un "Hard Gun" qui va dire seulement ce que tes données te donnent... ta réputation te suit.

Les experts développent ainsi toutes sortes de stratégies afin de faire profil bas, d'éviter la confrontation directe avec leurs superviseurs, voire de négocier une partie de leur intégrité pour assurer leur survie matérielle dans le champ. Par exemple, le manque de financement, la soumission aux impératifs gestionnaires et les besoins matériels des experts en poussent plusieurs à travailler sans être rémunérés parce que l'estimation du travail à accomplir ne correspond pas à la réalité et à la temporalité du terrain. Une pratique que Maurice affirme pratiquer :

Moi sérieusement, je mets le double des heures [en travail] par rapport aux heures pour lesquelles je suis payé, fait qu'il faut que t'aimes ça... Il faut limiter les attentes, tu peux pas me demander de regarder en détail tous les aspects du projet parce que ce qu'ils veulent c'est en avoir le plus possible! Mais là tu m'as donné tant de jours... Il y a un petit côté exploitation du consultant làdedans... [Les fonctionnaires qui demandent le travail] ils ont des journées de sept heures et demie admettons. Dans mon contrat c'est indiqué que je ne peux pas faire plus que sept heures et demie par jour : ça, ça veut dire que je ne peux pas collecter... ...... plus que sept heures et demie (rire) parce que c'est pas vrai [que tu fais juste ça quand] tu t'en vas visiter un projet, tu pars à six heures du matin, tu reviens à six heures le soir et ça, c'est tout le temps.

Ainsi, face à des demandes de performances irréalistes, certains trichent et bâclent le travail, d'autres plient et font des heures supplémentaires sans rémunérations dans le but de maintenir la relation qui leur garantit un gagne-pain. Pour Maurice, ceux qui exécutent les projets et les programmes se trouvent devant des exigences qui n'ont aucun lien avec ce qui est réalisable, mais relève davantage de l'exigence d'une performance d'efficience irréaliste de l'institution pour laquelle les fonctionnaires travaillent:

Trop souvent, les agences d'exécution, le gouvernement, son objectif c'est de toucher à beaucoup de trop de monde, d'avoir beaucoup trop de résultats par rapport à l'investissement qui est mis là- 
dedans. On vise beaucoup trop grand puis on a des prétentions face à ça comme si un petit pourcentage de budget allait changer le monde.

Pour Maurice, les dirigeants, les élites et les cadres des institutions du développement en viennent à se construire une réalité qui ne correspond pas à celle du terrain, au point où les experts, pour garder leur gagne-pain, en viennent à ne pas inclure dans leur rapport ce qui pourrait aller à l'encontre des idéologies favorisées par les dirigeants :

Tout le monde répète les mêmes choses, c'est comme dans n'importe quel domaine, dans les universités ou dans les hauts fonctionnaires, eux ils vont lire The Economist, bien l'autre lui, regarde le Journal de Montréal ce que tel gars a dit, ça répète toute la même chose. C'est ça qui devient la « réalité ». Bien l'autocensure, c'est ça : la ministre veut ça, puis si [les consultants], on est trop durs, on nous donnera plus de contrats, alors tout le monde se surveille.

Ainsi face aux diverses difficultés qu'il rencontre, l'expert en vient à développer des stratégies pour atteindre ses objectifs. Dans la section qui suit, nous examinerons ces stratégies de mise en marché de son expertise, d'adaptation au marché du travail et de communication.

\section{IV.5.a - L'expert stratège}

Officiellement, l'expert est chargé de construire ou d'appliquer des outils qui sont destinés à améliorer les conditions de vie des bénéficiaires. Dans les faits, l'expert doit aussi veiller sur ses propres conditions de vie, ce qui le pousse à adopter des comportements destinés à assurer sa survie dans ce champ en remplissant certains objectifs. Dans la prochaine section, nous exposerons les stratégies que les experts adoptent pour mettre en marché leur expertise, initier ou participer aux jeux de coulisse du développement et les activités de valorisation du développement qu'ils poursuivent. 


\section{La stratégie de l'étiquette de mise en marché}

Pour trouver du travail, certains experts utilisent des stratégies destinées à présenter leur expertise comme une «étiquette » de mise en marché qui confère la crédibilité et la légitimité d'œuvrer en développement international aux particuliers ou aux entreprises qui s'en revendiquent. Cette étiquette est utilisée pour simplifier la vente de leur force de travail et la compréhension de ce que celle-ci peut faire au profit des institutions de développement et des communautés. Pour Leonard, c'est un état de fait manifeste : «Dans le domaine de la consultation, le terme "expert" est aussi utilisé comme un outil de marketing: on dit que cette personne est une experte de ce domaine : c'est une personne fiable, compétente, professionnelle. Le terme est alors utilisé davantage comme une étiquette de mise en marché $\gg{ }^{18}$ celui qui s'en revendique offre ses services pour apporter un savoir nécessaire destiné à l'évaluation ou à l'élaboration d'organisations, de politiques ou de projets de développement international. Cette stratégie est dénoncée par la plupart des personnes rencontrées et les critiques sont articulées selon différents angles: appel à l'autorité, ambiguïté, connotation péjorative associée au terme. Leonard évite de se présenter comme un expert :

Je ne me suis jamais présenté ou qualifié comme un expert parce que ça me parait prétentieux et arrogant. Je préfère dire que j'ai de l'expérience dans certains domaines. Vous savez, j'ai travaillé dans plusieurs pays dans un domaine particulier, sur le même genre de dossiers. Vous voyez, je peux identifier le bon du mauvais, retenir les bons éléments et écarter les mauvais. ${ }^{19}$

Marie préfère plutôt substituer le terme spécialiste au terme expert, car : « [1]e terme expert, je ne l'aime pas parce que c'est comme se mettre un peu au-dessus de la mêlée, quelqu'un qui se prend pour un autre, alors je n'aime pas. » D'autres, comme Gérald présente plutôt un rejet de l'ambiguïté

18 Notre traduction: "In the counsulting field the term "expert" is also used a lot as a marketing tool : you say this person is an expert in this area : He's higly reliable. He's competent, professional. It's more of a "Label" thing I think. "

19 Notre traduction: "I never called myself an expert because it sounds a bit pretentious and arrogant. I'd rather say I've got experience in a certain area, you know I worked in many countries in a certain area, on the same things you know! I've got the good and the bad to compare to. To pull out the good things and push the bad things. » 
sémantique du terme « expert », qui est imprécis et facilite l'appel à l'autorité : « Ce terme expert est un fourre-tout général, car il n'est pas utilisé seulement en développement. Être expert, c'est canoniser un savoir sans que ça en soit nécessairement un. »

Pour Audrey et Gérald, le terme expert évoque d'abord et avant tout une connotation péjorative de certains comportements des experts en développement accusés de vivre et d'agir en vase clos, dans leur travail selon Audrey : «Dans la pratique de l'expertise, ce ne sont pas les connaissances ou l'expertise qui font la problématique, c'est sa pratique. Lorsqu'on fonctionne en vase clos de manière individuelle sans consulter les gens, on obtient des projets censés sur papier qui ne fonctionnent pas dans la réalité ». C'est vrai aussi dans leur implication avec la communauté qu'il souhaite aider. Ainsi, pour Gérald : «Certains experts préfèrent la vie "en vase clos" selon le train de vie occidental, les quartiers fermés et sécurisés et le repli identitaire. »"

On peut comprendre que le terme expert est connoté négativement. Les experts rencontrés souhaitent se détacher de la notion péjorative et des erreurs du passé associées aux experts. Ils souhaitent retenir la notion de reconnaissance des compétences qu'ils ont à apporter au développement quitte à adapter l'étiquette et à remplacer le terme expert par une autre appellation. Selon la situation et la personne à laquelle il s'adresse, l'expert se trouve ainsi à adapter son discours. Ainsi, le terme expert est reconnu par les personnes rencontrées comme un outil de mise en marché de sa capacité de travail, un terme qui est reconnu par les institutions du développement comme un gage decompétences. Or, le choix du terme expert pour s'insérer dans le champ et y travailler n'est pas la seule stratégie d'adaptation que l'expert adopte. De façon plus large, selon ses qualifications préalables ou encore selon ses motivations, l'expert choisit des stratégies pour se mettre en valeur et s'insérer dans le marché du travail du développement international. Dans la prochaine section, nous examinerons l'éventail de ces 
stratégies.

\section{S'adapter aux réalités du marché du travail d'expertise en développement international}

Pour trouver du travail, l'expert se doit de valoriser l'expertise en développement et la nécessité de produire des connaissances sur le développement. Maurice explique :

Bien, les experts, il faut qu'ils vivent et les experts, c'est souvent des universitaires [...] les universités sont pas toujours bien financées, il faut que tu cherches du financement un moment donné. Bien ça, t'en viens à vendre ta salade, à dire pourquoi c'est essentiel, toi ce que tu fais. Quand les gens qui ont besoin de toi sont en restrictions budgétaires, t'es obligé de dire là exactement, moi j'apporte ceci, cela qui est important.

Pour ce faire, les experts rencontrés adoptent ce que nous qualifions de stratégie d'approches du travail d'expert-consultant en développement international pour exposer ce qu'ils apportent comme valeur ajoutée à un projet ou à un programme. Ces approches ne sont pas mutuellement exclusives et peuvent être poursuivies en même temps ou consécutivement selon le contexte et le cheminement de la carrière de l'expert en développement international. Nous présentons dans cette section trois différentes stratégies adoptées par les experts que nous avons dégagés dans les entretiens, soit le « loup solitaire », l'« enrôlé » et le « coopérant volontaire ».

Le « loup solitaire » se présente comme un travailleur autonome, qui butine d'un endroit à l'autre pour se dénicher des contrats. Audrey décrit des experts qui « sont rémunérés pour leurs services et doivent pour ce faire, répondre à des appels d'offres des organisations internationales ou des entreprises. [...] Ces consultants travaillent en fonction d'un cahier des charges bien précis et évoluent autant dans le cadre de projets de développement international que national. [...] Ils ont un grand contrôle sur le travail qu'ils vont effectuer puisqu'ils travaillent en leur nom et œuvrent souvent sur de 
courtes missions. » Audrey amène à titre d'exemple : «Le programme des Nations Unies pour l'environnement (PNUE) fait appel à un consultant pour effectuer un séjour d'évaluation dans la région de Conakry afin de produire un rapport sur l'état d'avancement de la mise en place d'une aire protégée pour la pêche artisanale. » Cette stratégie a ses forces et ses faiblesses. Pour Leonard, c'est une excellente option pour « ne pas finir encore dans un poste de gestion [que de poursuivre] le travail de consultant à mon compte » elle permet d'être « son propre patron », d'éviter certaines responsabilités de gestionnaire. Par contre, comme le souligne Maurice, elle offre peu de sécurité matérielle, et dépend beaucoup du réseau professionnel de l'expert, car :

... c'est le fun être consultant, t'as pas de patron ou plusieurs patrons qui ne durent pas longtemps... ... mais un moment donné, oups, tous tes amis qui sont fonctionnaires commencent à prendre leur retraite, puis toi tu n'auras jamais cette chance-là. Ça en devient fatigant d'en entendre parler. J'ai trois de mes amis qui ont pris leur retraite [...] penses-y... ... ... Parce qu'un moment donné ça fait mal!

Pour Frank, le contrôle sur le travail n'est pas total parce que comme consultant indépendant : « souvent, t'as besoin d'ajuster ton rapport final par rapport à ce que le client veut. Si ça se trouve à être complètement opposé à tes valeurs et ton intégrité, bien, tu, comme on dit en bon français :"You pull the plug", puis tu dis : bien excuse-moi, mais je peux pas mettre mon nom sur ce rapport-là. »

En effet, l'expert consultant qui œuvre à son compte se trouve toujours à chercher l'équilibre entre ses besoins matériels et la réputation d'intégrité qu'il doit maintenir. De cette façon, ce choix de stratégie peut placer l'expert dans certaines positions de vulnérabilité matérielle lorsqu'il manque de contrat, ce à quoi certains experts répondent en changeant de stratégie d'approche du travail.

À l'opposé, d'autres préfèrent «s'enrôler dans une "boîte" ou une firme » de sous-traitance et y 
voient une option plus intéressante. D'après Audrey, ces derniers,

sont rémunérés pour leurs services et se voient dépositaires d'une partie d'un projet de solidarité internationale en leur propre nom. [...] Ce sont souvent des entreprises ou des institutions. [...] Par exemple : OXFAM international fait appel à une firme d'ingénieurs pour bâtir un four solaire au Mali pour un projet d'autonomisation financière des femmes sur 5 ans. Cette firme d'ingénieurs offre un service de consultant international au sens de "partenaire" dans le cadre d'un projet d'OXFAM.

Cette stratégie a ses forces. De l'avis de Leonard, s'enrôler permet d'extérioriser certaines tâches bureaucratiques qui sont extrêmement coûteuses en temps et en énergie lors de la rédaction des rapports :

Il faut faire des propositions, des budgets et de la planification qui lorsque faits par une seule personne peut être très prenant sur autre chose que l'évaluation elle-même. Les firmes ont un avantage sur ce plan dans le sens où elles sont plus efficientes dans le polissage des rapports (bibliographie, présentation, qualité de la langue, annexes, traduction), la gestion des finances et le remplissage de propositions, la planification des voyages, etc.

De plus, on peut y bâtir un réseau au dire de Leonard «Les firmes sont un lieu d'échange de l'expertise : on peut y consulter les collègues et avoir accès à plus d'information. » Sur le plan de la gestion de sa vie professionnelle, Maurice explique : « Il y en a plein [d'experts] qui sont consultant puis qui travaillent à l'intérieur de boîtes aussi... ils ont pas à chercher les contrats, c'est bon ça pour eux, certains aiment ça vivre avec ça. »

Aux yeux de Leonard, cette stratégie a aussi ses faiblesses. Par exemple, s'enrôler limite les considérations éthiques du développement à la logique du profit.

Les désavantages des firmes de consultants, c'est qu'elles doivent payer les factures alors elles doivent toujours ramener des contrats, ce qui veut dire qu'elles ne peuvent pas se permettre d'être difficile dans les contrats qu'elles font. Elles ne peuvent pas choisir que leurs sujets de prédilection 
ou seulement des organismes qu'elles aiment ou des missions dans lesquels elles croient. Elles sont froides en ce sens que tout est une occasion de travail pour ramener de l'argent qu'on ait la capacité de le faire avec qualité ou non. On tente, dans une firme, à maximiser les revenus.

Pour Maurice il s'agit d'un contexte qui limite la liberté d'action et de prise de position à travers son travail, car « ça te garde un patron par exemple! » En effet, le cadre gestionnaire impose une notion de productivité dont les contraintes de temps finissent par peser sur le plan personnel, et qui, au fil du temps, coupe l'expert de son sujet de travail, car le succès vous écarte du terrain selon Leonard :

Un des aspects négatifs de cette vie c'est que l'on va à tellement d'endroits, tellement souvent qu'après un bout, tu veux juste faire ton travail, aller à ta chambre d'hôtel, dormir, te lever et repartir à la maison. Alors j'ai eu plusieurs opportunités de voir beaucoup de choses extraordinaires, mais à un moment donné j'ai réalisé que tu ne peux vivre constamment comme un touriste, c'était le mauvais côté de ce genre de vie. [...] Le reste du temps, je le passais à l'aéroport entre deux vols, dans ma chambre d'hôtel à rédiger mes rapports pour être à la maison au plus vite. Être sur le terrain est super. Dans mes premières années, je faisais plus de travail sur le terrain, mais en grimpant dans des postes de senior, il y avait de moins en moins de terrain et davantage de travail avec les représentants du gouvernement sur les problèmes rencontrés. Je regrette d'avoir fait ce choix de carrière, c'était beaucoup moins intéressant.

Cette période dont Leonard se fait nostalgique, c'est celle où il œuvrait lui-même dans un autre cadre d'expertise plus « près » du terrain, là où les justifications du choix de stratégie de travail sont davantage morales que pécuniaires.

Les experts qui choisissent cette stratégie évoquent des motivations éthiques derrière le choix d'opérer comme « coopérant volontaire », un type de consultant qui travaille principalement avec des organismes sans but lucratif internationaux et locaux de solidarité ou de coopération internationale. À ce propos, Audrey décrit un « expert » qui

... est recruté pour prendre part à un projet des organisations de solidarité internationale. [...] En général, ces consultants viennent "renforcer les capacités" d'acteurs déjà impliqués dans un projet de développement à long terme et leur implication s'inscrit souvent dans un cadre de mission de 
plusieurs mois. [...] Ces consultants ne sont pas rémunérés pour leur travail, mais bénéficient d'avantages sociaux et de per diem pour leur subsistance à même les fonds des organismes de solidarité internationale. Ils sont autonomes, mais doivent rendre compte de leur travail constamment puisqu'ils ne travaillent pas en leur nom propre. [...] L'organisme SACO fait appel à un volontaire pour appuyer le Ministère de la Condition féminine d'Haïti dans la mise en place d'un système de gestion de conflit organisationnelle dans le cadre d'un projet plus large de redressement de la gouvernance locale.

Or, une telle stratégie opte pour une situation financière encore plus précaire. « J'ai 30 ans et je vois mes amis d'université qui sont casés qui commencent à avoir des enfants, qui ont déjà acheté leur première maison... je n'ai rien de tout ça! » Par contre, pour Leonard, elle offre une occasion hors du commun d'accumuler une expérience de terrain riche en apprentissages qui ont moins lieu lorsqu'on travaille pour les firmes :

Ce que je préférais c'est quand on sortait sur le terrain, à travailler avec des gens du pays, dans des coins que les touristes ne visitent jamais. Tu en viens à savoir comment les gens vivent, à connaitre un brin de leur réalité. Ce sont de pays vraiment pauvres, souvent avec des bidonvilles et tout. C'est beaucoup plus riche comme expérience.

On notera ici que Leonard tient en haute estime l'expérience du terrain acquise comme coopérant volontaire à l'image de la vaste majorité des autres experts rencontrés. Or, les conditions de travail des coopérants volontaires sont souvent les moins bonnes. Ce qui en fait une porte d'entrée ou encore l'endroit où l'on va ponctuellement mettre à jour ses expériences.

Ainsi selon leurs convictions et leurs qualifications, certains choisiront de s'enrôler dans une firme pour s'insérer dans le champ du développement alors que d'autres choisiront l'implication comme coopérant volontaire. Le loup solitaire profitera des expériences acquises dans ces situations et des contacts qu'il y a noués pour s'affranchir des obligations qui viennent avec les deux stratégies précédentes. Or la stratégie d'insertion priorisée par chacun aura une influence sur les stratégies de communication de l'expert avec ses partenaires de travail. 


\section{Communiquer des messages et des recommandations}

En fonction de la personne ou l'institution à qui ils s'adressent, les experts rencontrés adaptent leur message. Lorsqu'il s'adresse aux agences, Maurice affirme que la plupart du temps, à l'image des fonctionnaires, il opte pour l'autocensure et la conformité avec les positions idéologiques du gouvernement afin de maintenir ses chances d'obtenir un nouveau contrat :

Le consultant évaluateur a beaucoup moins d'espace qu'avant de réflexion et puis d'impact. On te dit : «Limite-toi à ça, puis si tu touches à un champ qui n'est pas de tes affaires, on va te le dire.» $\mathrm{Tu}$ viens que tu le sais, puis tu sais que dans la vie là il y a pire que la censure, il y a l'autocensure! (rire) puis quand t'es rendu là ça commence à mal aller puis veut, veut pas, si tu en veux d'autres contrats...... et bien tout ça est là [...] Dans l'ambiance, l'autocensure, c'est pas juste pour les fonctionnaires. Les fonctionnaires ils en posent pas de question. Ils appellent ça le second guessing, là [ce qu'ils pensent que la] ministre veut voir ou ne veut pas voir [dans un programme]. Finalement, ils s'autocensurent tout le temps, tout le temps, ils vont écrire comme ils pensent qu'elle pense; elle veut entendre parler de tel sujet, mais pas de celui-là... Bien là, tu finis par t'autocensurer, tout le climat est un peu de même. Moi j'irai pas m'en prendre au projet pour le détruire parce qu'on va se dire celui-là il est pas tenable...

Pour les mêmes raisons, Maurice évolue dans les coulisses des instances officielles, car « Il y a beaucoup de fonctionnaires qui ne veulent pas discuter directement avec toi par courriel ou autre. Ils vont utiliser d'autres moyens non surveillés, pour s'assurer que la hiérarchie ne puisse pas faire un audit de leurs courriels qui mènerait à leur renvoi.» D'autres experts vont faire avancer des recommandations en dehors des canaux officiels, hors de vue des mécanismes de surveillance des grands organismes et des employeurs. Maurice explique : «Dans le rôle d'agent de suivi et d'évaluateur, tes objectifs sont très clairs et très précis. Des fois, tu peux parler de ça avec les chargés de projet sans l'écrire aussi. Si ça fait partie des questions, tu peux l'amener dans des conclusions. » Pour Gérald les coulisses font partie intégrante des outils formels et informels, officiels et officieux de l'expert pour faire adopter des recommandations. 
Pour ma part j'opte pour trois niveaux de stratégies afin de faire mettre en place une recommandation: d'abord, être là en personne et regarder comment les gens travaillent, ensuite discuter des idées officieusement avec les gens sur place en suggérant des idées indirectement aux dirigeants comme au travailleur de base, pour terminer, si le message ne passe toujours pas, je fais des recommandations par la voie officielle soit par la discussion officielle ou dans le bon vieux rapport.

Pour se conformer et éviter de saboter son poste ou sa carrière, l'expert opte ainsi de passer des messages avec un minimum de risque, de laisser des traces lorsque le propos est sensible et pourrait mettre en péril la relation de l'expert avec son employeur ou encore avec ses collègues.

En effet, la communication est l'élément par lequel passe chacune des actions de l'expert, ainsi le choix des mots et le langage adopté occupent une place prépondérante dans les activités de l'expert ce qui permet d'identifier les stratégies auxquelles les experts ont recours pour faire face aux défis qu'ils affrontent. Ainsi, avec le recours à l'étiquette d'expert, en alternant les différentes façons de chercher du travail, ils démontrent qu'une partie importante de l'expertise comporte une maitrise des rhétoriques qui y sont associées.

Dans cette section à propos des situations d'expertise rencontrées, nous avons examiné plus étroitement ce que fait l'expert dans son travail de tous les jours grâce à l'examen de l'éventail des tâches qu'il estime effectuer pour le développement telles que les recommandations, les évaluations et suivis de projet, la conception et la mise en place de projet ou encore le temps consacré à l'exécution de ses tâches bureaucratiques. Par ailleurs, nous avons aussi sondé les réflexions des experts rencontrés pour faire une recension des plus grandes craintes, de la pression à la production issue du cadre gestionnaire que certains d'entre eux estiment subir et des risques que les experts sont appelés à contrôler. En dernier lieu, nous avons examiné comment adopter l'étiquette d'expert, adapter son discours au destinataire et varier sa stratégie d'insertion dans le champ permet de tirer profit de la 
situation.

Dans cette recherche auprès de sept experts du développement international, nous avons abordé avec ces derniers les diverses qualités, caractéristiques et compétences qu'ils considèrent comme caractères impératifs d'une expertise véritable et légitime en développement international. En second lieu, nous avons examiné la perception de l'expert à propos du travail d'expertise avec l'examen des catégories qu'il associe à son propre travail et à celui de ses confrères. Par la suite, nous avons présenté l'ambiance qui règne au sein du champ de l'expertise en s'interrogeant sur les débats internes à propos de l'objectivité de l'expertise et les qualifications. En dernier lieu, nous avons évoqué la rencontre de ces éléments dans différente situations, soit dans les tâches quotidiennes ou encore dans les réflexions au sujet des travers redoutés, et finalement commenté les stratégies déployées par les experts pour maintenir leur place dans le champ.

Forts du témoignage de ces experts, nous sommes maintenant en mesure d'analyser les données produites par la synthèse des entretiens dans le but de tracer une esquisse du champ de l'expertise en développement international. Cette esquisse nous permettra de tisser des liens entre les différents discours tenus par les experts rencontrés et de cette façon identifier comment « on devient expert » en développement international, plus spécifiquement comment ils pratiquent l'expertise en développement, comment ils interagissent entre eux et avec les communau tés visées par le développement, comment s'établit la « légitimité » d'une expertise en développement international et dans quelles situations on fait appel à eux. 


\section{CHAPITRE V - ANALYSE - LE CHAMP DE L'EXPERTISE EN DÉVELOPPEMENT INTERNATIONAL}

À partir des résultats présentés dans le chapitre précédent, nous avons mobilisé le cadre théorique bourdieusien présenté au chapitre II afin de répondre à nos questions de recherche. Pour ce faire nous allons dans un premier temps, identifier les capitaux en jeu dans le champ de l'expertise en développement international, puis examiner, la nature des mouvements de capitaux à travers les rapports du champ de l'expertise avec les champs extérieurs. En dernier lieu, nous analyserons le champ de l'expertise comme microcosme social, avec ses règles, ses frontières et son illusio. Cet examen des résultats à travers le cadre d'analyse proposé nous permettra de déterminer par quels mécanismes, expériences et parcours on devient expert en développement international.

\section{V.1 - Structure des capitaux spécifiques}

Le champ de l'expertise est un lieu où le capital économique n'est pas l'enjeu principal des luttes qui y ont cours. Si le capital économique structure de manière importante le champ de l'extérieur, à l'intérieur du champ de l'expertise en développement plusieurs formes de capital culturel spécifique ont une valeur symbolique singulière. De la même façon, les relations sociales se construisent en fonction de la valeur perçue des formes de capital symbolique valorisées. Dans la section qui suit, nous identifierons ces capitaux, leur composition et leurs caractéristiques spécifiques. 


\section{V.1.a - Capital technologique}

Le capital technologique est un capital que les experts accumulent par leurs expériences et leurs formations qu'ils ont poursuivies au cours de leur carrière. Il est composé principalement des capitaux scientifiques, académiques et techniques acquis par les experts et étant à leur disposition. Ici, on pourrait paraphraser Bourdieu (Bourdieu 1997a, 52): «Le capital technologique est le portefeuille de ressources scientifiques (potentiel de recherche) ou de techniques différentielles (procédés, aptitudes, routines et savoir-faire uniques et cohérents, propres à diminuer la dépense en main-d'œuvre ou en capital ou à augmenter le rendement) susceptibles d'être mises en œuvre dans la conception » et la fabrication du développement.

Le capital technologique possède deux caractéristiques spécifiques. Premièrement, le capital technologique est un capital culturel incorporé, c'est-à-dire qu'il est acquis à partir d'apprentissages qui permettent à ceux qui y consacrent le temps d'intégrer des compétences spécifiques. Ainsi, les experts rencontrés ont tous poursuivi des études universitaires de cycles supérieurs. Or, l'ensemble de ces apprentissages regroupant souvent un capital scientifique et technique important acquis dans un parcours universitaire ne suffisent pas à eux seuls pour être un expert reconnu. En effet, le parcours professionnel occupe une place importante dans l'accumulation des capitaux de cette forme. Les experts y développent des savoir-faire et des savoir-agir qu'ils doivent appliquer dans différents contextes culturels. À travers ces parcours, les agents développent des techniques de communications pour pouvoir répondre aux exigences élevées du champ.

En second lieu, le capital technologique est périssable puisque certaines connaissances liées entre autres à la connaissance des programmes et certaines techniques de rédaction administrative évoluent 
dans le temps, ce qui fait que des personnes retraitées comme Carl nous avertissent qu'après trois ans d'inactivité à l'étranger, il se considère lui-même comme « rouillé » malgré des décennies d'expérience dans le domaine. Ces agents retraités ont ainsi perdu le contact avec les meilleures pratiques actuelles, ne connaissent pas l'étendue des changements de politique en vigueur pour les demandes de financement et ont des lacunes au niveau de la connaissance de l'actualité scientifique de leur domaine. Ceci dit, pour Gérald, ces connaissances peuvent être maintenues par l'activité et le suivi des actualités scientifiques de son domaine de spécialisation. Gérald explique que malgré son parcours académique aux cycles supérieurs, un collègue possédant un baccalauréat, travaillant en développement international et s'informant des plus récentes actualités scientifiques en développement possède, à son avis, un niveau de capital scientifique et technique équivalent au sien puisqu'il n'a que répété cet exercice dans un cadre académique reconnu par des institutions universitaires sans nécessairement être aussi près du terrain. De cette façon, un collègue au capital académique inférieur qui suit le rythme de production des connaissances, est en mesure de maintenir le volume symbolique de son capital technologique s'il est en mesure de l'associer à un parcours professionnel adéquat tout en rendant cette quantité de capital appréciable par le nombre d'épisode d'acquisition qu'il est en mesure de démontrer.

\section{V.1.b - Capital organisationnel}

Le capital organisationnel se caractérise par le fait qu'il est institutionnalisé et qu'il n'est pas directement possédé par l'expert. C'est un capital qui est généré en dehors de l'expert, c'est-à-dire que celui-ci n'y a accès que lorsqu'il travaille pour un organisme donné ou qu'il en est le propriétaire direct. Ce capital garantit des moyens que l'expert lui-même n'est pas en mesure d'apporter individuellement. Il existe deux formes de capital organisationnel : le capital organisationnel de localisation et le capital organisationnel financier. 
Tel que nous l'avons constaté dans notre recherche, le capital financier s'acquiert par l'accès aux contrats gouvernementaux, ou encore par l'accès aux postes de coopérants volontaires ou de gestionnaires de programmes. Ce capital financier permet de se déplacer, de subvenir à ses besoins ou encore de mobiliser une bureaucratie pour faire face aux appels d'offres lorsque l'expert est employé, partenaire, voire dans de rare cas, propriétaire d'une firme. Elle lui permet de libérer du temps consacré aux tâches bureaucratiques pour se concentrer à l'accomplissement de ses tâches d'analyse, d'évaluation et de rédaction de rapport. À titre d'exemple, Audrey décrit précisément le genre d'expert qui jouit de ce capital sans pour autant le posséder lorsqu'elle décrit « Ces consultants [qui] ne sont pas rémunérés pour leur travail, mais [qui] bénéficient d'avantages sociaux et de per diem pour leur subsistance à même les fonds des organismes de solidarité internationale ». La plupart des experts rencontrés avaient ainsi un accès à des capitaux financiers qu'ils contrôlaient plus ou moins pour leur subsistance grâce aux contrats ou aux mandats qu'on leur accorde.

Dans sa seconde forme, le capital organisationnel prend celle d'un capital de localisation constituant l'ensemble des connaissances de codes et rouages spécifiques des organisations, des communautés ou de l'État que l'expert est amené à connaître de par sa position sociale et sa localisation géographique. À ce titre, le fait d'être assigné à une ville, un pays ou une région particulière est généralement issu d'une occasion de travailler offerte par l'État, par une institution ou un organisme dont l'expert n'est pas propriétaire. Il y est assigné ou choisi parmi les offres d'assignation qui sont disponibles ou pour lesquelles un poste ou un contrat lui est attribué.

\section{V.1.c - Capital social}

Le capital social d'un agent correspond à l'ensemble des relations actuelles ou potentielles reliées 
à un réseau durable de relations ou un groupe. Ces relations sont entretenues par des échanges matériels et symboliques. L'accès à un capital organisationnel permet souvent à un agent d'accroitre son réseau potentiel et de nouer de nouvelles relations.

Ces relations débutent et se poursuivent généralement dans des lieux et des contextes particuliers. Comme nous l'avons constaté dans le cas du champ de l'expertise en développement international, il est clair pour Marie et Gérald que les experts socialisent entre eux et avec les agents du champ des expatriés à l'occasion de simples rencontres, de soirées thématiques ou de fêtes dans des lieux symboliques et institutionnels, tels des quartiers, des ambassades, le centre culturel francophone, le lycée français local, les associations, et clubs, ou encore dans des lieux de pratiques culturelles spécifiques tels les salles de sports de jeux et de cérémonies, restaurants, boutiques et supermarchés. Ces occasions constituent des moments privilégiés où la camaraderie et les relations qui s'y nouent sont souvent utilisées par la suite dans le cadre professionnel pour échanger différentes formes de capitaux.

Sur le plan spécifique, le capital social se caractérise par le fait qu'à l'image du capital technologique, il est indirectement périssable, car lié intimement à la capacité de mobiliser du capital organisationnel, c'est-à-dire que le capital social est tributaire des aléas professionnels et du maintien des relations entre les agents. Au fil du temps, des agents finissent par quitter le champ, d'autres changent d'organisation et plus souvent qu'autrement les agents changent d'affectation. Au gré de ces mouvements d'agents, le réseau social des agents tend à se transformer en fonction des capitaux qu'ils sont en mesure de mobiliser pour maintenir leurs relations. Ainsi, le capital social permet à celui-ci d'utiliser les capitaux technologiques et organisationnels à sa disposition pour générer une forme de capital symbolique spécifique au champ et qui en constitue l'enjeu principal : le capital d'expertise. 


\section{V.1.d - Capital symbolique propre au champ: le capital d'expertise}

Le capital symbolique du champ est la principale monnaie d'échange des experts. Le capital symbolique de l'expert en développement correspond à la quantité de capital technologique, organisationnel et social qu'il est capable de transformer en capital d'authenticité, d'autorité, et d'expérience. Cette capacité à transformer ce capital permet de s'afficher comme un expert indépendant, impartial et légitime.

Le capital d'authenticité correspond à cette capacité implicite et intuitive de déterminer grâce à son habitus, quels informations et outils techniques sont mieux adaptés à la tâche demandée et lesquels seront pris au sérieux, acceptés, voire valorisés par l'agent qui recevra l'avis de l'expert. Par exemple, la connaissance des cadres de références attendus dans les rapports d'évaluation d'un projet de développement demandé par l'État canadien, ou encore les façons de présenter des recommandations à un organisme local sans passer par les canaux formels tel que le décrivait Gérald ou encore cette capacité de participer aux apprentissages de l'ingénierie inverse du processus d'évaluation.

Le capital d'autorité est un cumul de la possession d'expériences à l'étranger, d'expériences de développement, de capital organisationnel et de diplômes académiques dans des disciplines reconnues. Ce capital est en partie objectivé par le curriculum de l'expert. Il confère un statut auquel l'expert peut faire appel pour sanctionner le développement. Un exemple d'utilisation du capital d'autorité est le capital utilisé par les experts ayant plus de séniorité ou les « experts seniors » décrits par Leonard devant «trouver des consultants plus jeunes avec moins d'expérience qui feront le travail pour moins cher ». Dans de telles situations, ces experts seniors se servent de leurs gestes antérieurs pour avoir un minimum d'implication dans le processus, où ils sanctionnent et supervisent en même temps les 
expertises effectuées sous leur direction. Les experts « au bas » de cette hiérarchie sont dits « juniors » parce qu'ils possèdent moins d'expériences donc un capital d'autorité inférieur.

En dernier lieu, le capital d'expertise est formé du capital d'expérience, un capital objectivé, cumulable dans le temps mais qui perd de la valeur graduellement lorsque le contact avec le terrain est perdu. Les experts rencontrés s'efforcent donc activement à maintenir sa valeur tant en conservant dans leurs activités de travail des séjours et des liens de travail à l'étranger. Selon eux, ce capital permet d'avoir accès plus aisément au champ de l'expertise en développement grâce à l'expérience du monde non-occidental. Son accumulation permet de maintenir, voire d'augmenter son statut. Cette expérience est un capital rare qui dans plusieurs cas est valorisé davantage que le capital académique et entre dans la composition des trois autres composantes du capital d'expertise.

Les experts habilités à transformer leurs capitaux technologiques, organisationnels et sociaux dans une ou plusieurs de ces formes de capital d'expertise sont en mesure de mobiliser contre d'autres capitaux à transformer ou contre du capital économique tel un salaire qu'ils réinvestissent souvent dans l'acquisition de capitaux académique et technique tels des formations et diplômes dans le but d'accéder à des postes plus rémunérateur en terme d'expériences ou de capital économique. On peut imaginer dans un exemple fictif, qu'en postulant pour un poste de coopérant volontaire dans un organisme au Libéria, un expert fera valoir ses expériences précédentes dans des postes similaires au Ghana, en Gambie et au Sénégal. Il fera aussi valoir les compétences technologiques requises par le poste et aura peut-être accès en primeur à ces dernières grâce à un collègue qu'il a rencontré pendant une soirée à l'ambassade du Canada à Dakar et dont il a soigné la relation.

Cette courte énumération des capitaux d'importance dans le champ de l'expertise nous permet 
donc d'affirmer deux choses. Tout d'abord, les capitaux pris individuellement ne constituent pas en soi l'expertise en développement international. C'est plutôt la combinaison de plusieurs capitaux différents qui permet à un individu de devenir expert. Deuxièmement, tous les experts ne sont pas égaux. En effet, les combinaisons de capitaux possédés sont multiples et varient en fonction de la valeur fluctuante accordée à chaque capital. En ce sens, nos résultats démontrent qu'il existe des inégalités en termes de capitaux possédés par les experts en développement international. Ces inégalités sont produites par la relation avec l'extérieur du champ du développement international, le niveau d'intégration des règles du milieu de même que l'habileté de l'expert à utiliser son capital à son profit.

\section{V.2 - Le rapport avec l'extérieur du champ}

Au plan macrosocial, le champ de l'expertise en développement international entretient de nombreuses relations avec d'autres champs, notamment, le champ bureaucratique, le champ du pouvoir dont les agents institutionnels principaux au Canada sont l'ACDI et le ministère des Affaires étrangères, et le champ des expatriés qui constitue l'une des principales sources de capital social en dehors du champ de l'expertise lui-même. Dans les sections suivantes, nous examinerons succinctement la nature de l'influence de ces champs et de leur matérialisation dans le champ des experts en développement international.

\section{V.2.a - Le champ bureaucratique (ACDI, MAECD)}

Selon les agents rencontrés, parmi les champs qui ont une influence importante sur le champ de l'expertise canadienne en développement international, le champ bureaucratique de l'État canadien est de loin le plus influent. Les institutions telles que l'Agence canadienne de développement international (ACDI) et le ministère des Affaires étrangères, du commerce et du développement (MAECD), cadrent 
la majeure partie des demandes d'expertise à travers plusieurs mécanismes. Tout d'abord, ce sont ces institutions qui demandent l' « évaluation-suivi », qui mettent en place des appels d'offre en matière de projet de développement et qui financent ces derniers. Pour nombre de ces mécanismes, le financement est associé à une manière de faire l'évaluation-suivi, de mettre en place un projet et de sous-traiter le travail demandé. Ainsi, le champ bureaucratique est l'une des sources principales de capital financier, il est en quelque sorte l'espace spécialisé dans l'activité administrative du champ du pouvoir.

\section{V.2.b - Le champ du pouvoir}

Si elle est plus diffuse et moins facilement perceptible sur le terrain, l'influence du champ du pouvoir est considérable sur le champ de l'expertise en développement international canadien. Ainsi, le gouvernement conservateur du premier ministre du Canada, Stephen Harper et les divers ministres qui ont été responsables des enveloppes liées au développement sont identifiés par les experts rencontrés comme des éléments d'influence du champ du pouvoir sur la bureaucratie canadienne du développement. Ces personnes rencontrées font un lien direct entre les personnalités et les affiliations politiques des responsables de l'aide canadienne. C'est-à-dire qu'ils remarquent une plus grande

politisation de l'aide au développement par la domination actuelle de la justification idéologique conservatrice au détriment de l'utilisation de l'expertise comme outil de légitimation et de justification principal du développement pour les organisations bureaucratiques canadiennes. Ainsi, le champ du pouvoir se montre prêt à fournir un capital organisationnel principalement financier en échange des justifications qu'il estime pertinente.

\section{V.2.c - Le champ des expatriés}

Le champ de l'expertise en développement international en Afrique est un sous-champ du champ 
des expatriés occidentaux, c'est-à-dire de travailleurs de tout acabit qui immigrent en Afrique dans le but d'y travailler. Certains le font de manière temporaire, c'est-à-dire qu'ils n'ont pas l'intention de s'y établir en permanence et qu'ils ont l'intention de retourner vivre au Canada. D'autres y sont à plus long terme sans plan précis.

Le champ des expatriés possède une charge symbolique forte. En fonction de la force de sa présence dans une ville, ce champ possède souvent ses propres quartiers, ses marchés, ses restaurants, ses hébergements hôteliers et ses institutions que fréquentent assidument plusieurs experts-consultants comme l'évoque Marie :

Les consultants classiques se retrouvent souvent aux mêmes endroits sur le terrain, dans les mêmes établissements, restaurants, bars, hôtels coupés des populations. Surtout en Afrique, ces comportements sont encouragés par les ambassades qui y organisent fêtes, banquets, réunions et soirées de rencontre. C'est une structure entraînante par le luxe qu'elle affiche. Cela crée un monde parallèle.

Le champ des expatriés constitue un lieu de rencontre où experts-consultants, diplomates et autres agents canadiens et occidentaux se rencontrent pour échanger à l'écart de la population locale (entre occidentaux.) On peut y accumuler du capital social en nouant des relations avec les agents de l'État canadien, y trouver des lieux qui font référence à la culture occidentale « internationale » ainsi qu'y trouver d'autres experts et collègues expatriés. C'est une sorte d'espace culturel et temporel occidental à l'extérieur de son espace géographique habituel. Les règles de la vie occidentale s'y appliquent et la culture dominante est fortement influencée par le champ bureaucratique de l'État canadien et des autres États occidentaux influents et présents dans la région tels la France, la GrandeBretagne ou les États-Unis d'Amérique. 
Conséquemment, ce champ exerce son influence la plus importante sur le capital social des experts. En effet, l'appartenance à ce champ permet à certains de rafler des contrats ou encore d'obtenir des informations importantes dont on peut tirer un profit personnel ou organisationnel par la fréquentation des lieux de rencontres des expatriés. Par ailleurs, il permet à d'autres de maintenir un lien avec la culture occidentale, son évolution et ses tendances au niveau du développement international et d'entretenir des relations avec les autres agents de la diplomatie canadienne et occidentale.

En second lieu, nombre d'experts puisent dans leur appartenance à ce champ un capital de légitimité et d'autorité rattachés à la domination de la culture occidentale. Ces capitaux dont la valeur fluctue d'un champ à l'autre et parfois les habitus acquis par les agents à titre d'experts dans le champ de l'expertise en développement international peuvent être transposables et utilisables dans d'autres activités, par exemple s'ils veulent travailler pour une entreprise, pour un organisme philanthropique, en recherche universitaire, ou encore en diplomatie internationale pour ne donner que quelques exemples.

En somme, les experts en développement international sont les agents qui permettent aux capitaux de passer d'un champ à l'autre et de transformer la symbolique qui leur est rattachée. Ainsi, le champ bureaucratique est une source importante, presque indispensable, de capital financier pour les experts, alors que le champ des expatriés constitue un lieu important dans la construction du réseau social et, finalement, le champ du pouvoir est une source de capitaux variés et en quantité abondante. 


\section{V.3 - Le champ de l'expertise comme microcosme social}

Le champ de l'expertise en développement international est un sous-champ du développement international qui possède ses propres dimensions socialement structurées. Il possède sa propre structure de capitaux, ses frontières, un rapport particulier avec les autres champs et des règles et des luttes qui lui sont propres. Ces caractéristiques seront examinées dans les paragraphes suivants.

\section{V.3.a - Frontières}

Pour procéder à leurs activités et conserver leur légitimité, les experts se disputent des capitaux à l'intérieur de frontières bien précises qui comportent trois délimitations distinctes de nature technique, géographique et conceptuelle.

Dans un premier temps, la limite est technique, c'est-à-dire il faut être en mesure d'apporter, d'appliquer et de discuter des résultats à partir des méthodes scientifiques et gestionnaires approuvées par le monde académique et bureaucratique occidental. Ainsi, il est impossible de percer ce champ du développement international et de se voir accorder une crédibilité ou une légitimité sans proposer ou utiliser de telles méthodes ou encore sans maîtriser les cadres d'évaluation déterminés par les agents de financement ou sans avoir passé minimalement par un parcours académique offert par une institution occidentale.

En second lieu, la limite est géographique, dans le sens où l'expertise doit être pratiquée à l'extérieur du cadre culturel occidental (mais paradoxalement, principalement dans ses anciennes

colonies). L'expertise est alors invoquée dans des situations où des agents, principalement des États occidentaux, jugent nécessaire de superviser le transfert de capitaux entre les champs bureaucratiques 
étatiques ou nongouvernementaux de l'occident à l'espace social africain, comme le démontre le nombre important d'experts rencontrés qui estiment pratiquer le style du « traducteur » entre organismes dits « locaux » et organismes dits « internationaux » en lien plus ou moins direct avec des acteurs étatiques occidentaux.

Pour terminer, cette limite est conceptuelle. L'appartenance au champ de l'expertise en développement international consiste en un dépassement du stade de l'opinion, soit d'être en mesure d'appuyer ses dires avec des données et une évaluation concrète dans un cadre scientifique. Lorsque les interactions ont lieu en dehors de ce cadre, on se situe à l'extérieur du champ, ce qui permet à Maurice d'évoquer le spectre de voir les histoires d'horreurs se répéter :

«Est-ce que les experts peuvent nuire au développement international?» Non, c'est le contraire. C'est les gens qui connaissent pas ça [le] développement international [qui peuvent lui nuire]. Tout le monde connait ça, tout le monde a le droit à son opinion là-dessus [...] c'est le manque d'experts qui doit être vu comme le plus dangereux pour moi, pour les pays en développement, plutôt que le contraire.

De cette façon, le développement ne peut exister sans une sanction experte ce qui suppose qu'avant la sanction, une définition universelle du développement est déterminée par l'agent (expert, bailleur de fond, ONG, État, OI) qui définit les critères du développement. C'est aussi ce qui rend l'expertise légitime et qui lui donne son caractère essentiel. À l'intérieur de ces frontières, suivant des règles plus ou moins précises, les experts se disputent un capital culturel spécifique qui porte une charge symbolique propre au champ du développement international que seul celui-ci est en mesure de générer. Conséquemment, il est impossible de devenir expert sans œuvrer à l'intérieur de ces frontières sans respecter les règles qui régissent cet espace. En ce sens, ces frontières ont un effet structurant important dans la composition des règles du champ de l'expertise en développement international examinées dans la section suivante. 


\section{V.3.b - Les règles}

Au-delà des éléments plus palpables que sont les capitaux et leurs interactions, le champ de l'expertise en développement international possède des règles consacrées qui contraignent toutes les dimensions subjectives du champ et la structure de son capital, au rang desquels on trouve le droit d'entrée, les doxa et l'illusio. La quantité de capitaux possédée par l'expert et le degré d'habileté avec lequel il est capable d'utiliser les règles du champ à son profit détermine sa position en tant qu'expert dans le champ.

\section{Le droit d'entrée}

Ne s'improvise pas expert en développement qui veut. Les impératifs de l'expertise ${ }^{20}$ nous renseignent sur ce qui correspond aux «prix » d'entrée du champ que tous reconnaissent posséder ou reconnaissent nécessaire pour entrer dans le champ, qu'ils se revendiquent ou non de « l'appellation » d'expert $^{21}$. Ainsi, l'expert doit au minimum avoir participé à un processus de développement et y avoir acquis une socialisation à ces codes et posséder un capital académique pour pouvoir y entrer. La plupart des experts rencontrés ont fait cette socialisation en participant à un projet, à une mission ou à un stage d'initiation au développement, à la coopération ou à la solidarité internationale avec un organisme reconnu. Leur parcours se poursuit lorsqu'ils prennent ensuite en charge une tâche spécifique dans un projet de développement et ont une reconnaissance dans une discipline académique reliée aux sciences de la gestion ou aux sciences sociales. Cette combinaison de connaissances et d'expériences suffit généralement à pouvoir entrer dans le champ de l'expertise en développement. À travers ce processus d'entrée, le champ tend à sélectionner les agents qui ont un habitus de base approprié. Cependant pour

20 Voir la section V.1 dans le Chapitre Présentation des résultats à la p.55

21 À ce sujet, la section Le champ de l'expertise : changement de régime et enjeux de lutte à la p. 135 explore davantage les implications derrière ce refus de se définir comme « expert». 
maintenir sa place dans le champ, il faut être en mesure de développer un habitus expert plus élaboré que nous examinerons dans la section qui suit.

\section{L'habitus expert: les critères privilégiés}

Pour pouvoir œuvrer en développement et pour que le champ s’intéresse à lui, un agent doit avoir au minimum un habitus précis qui, selon les personnes rencontrées, comporte deux caractéristiques minimales, soit le fait d'avoir vécu « là-bas » et la capacité de développer une rationalité argumentative scientifique.

Tout d'abord, «Si tous les experts avaient vécu là-bas avec une "piasse" par jour, là ils pourraient peut-être mieux comprendre. L'idéal c'est d'être près du terrain pour comprendre ses logiques et comment on peut intervenir dans ces logiques » exposait Gérald. En effet, au cœur de cette affirmation, il souligne l'importance d'acquérir par une socialisation avec les personnes visées par le développement, la connaissance matérielle des conditions de vie des communautés. Les expériences acquises dans ces situations permettent à l'expert d'appréhender la complexité des enjeux et des

problématiques dans lesquelles il est appelé à agir. Ces expériences ne peuvent être acquises que si l'expert effectue des séjours à l'étranger dans des communautés ciblées par le développement, à l'extérieur des cercles touristiques, ce que la plupart qualifiera de « terrain ».

En fonction de la position sociale de l'expert, la forme de ces séjours peut varier énormément tout dépendant de la forme de capital symbolique que l'expert est en mesure de mobiliser. Dans chaque cas, cet habitus est acquis soit par la socialisation avec les collègues et supérieurs dans le cas d'assignation professionnelle, soit formellement dans le cadre de formations offertes par les responsables de stage 
dans le cadre de stages étudiants de coopération à l'étranger, ou encore par le biais d'un ami ou d'un membre de la famille dans le cas d'une socialisation par la famille ou les relations sociales. Un nombre élevé de terrains abordés suppose, que ce soit le cas ou non, que l'expert est en mesure de vivre à l'extérieur du monde occidental, soit à l'extérieur des frontières géographiques des États occidentaux, de s'intégrer à une communauté qui lui est étrangère, de saisir les règles des institutions locales et des champs locaux dans lesquels il pourrait être parachuté. Dans les mots de Marie, ce bagage « permet d'apporter le recul » qu'on attend de l'expert, soit un processus d'apprentissage par l'action qui, à terme, lui permet de maitriser cette capacité d'avoir les interactions adéquates parmi les choix possibles d'interprétation des situations qui s'offrent à lui dans un environnement de développement international. Les habiletés acquises lors des séjours à l'étranger dans un cadre de coopération occupent une place importante dans les habitus valorisés par le champ de l'expertise. À ce titre, elle vient à parité avec la maîtrise des outils et des discours scientifiques.

Enfin, pour beaucoup des experts rencontrés dans le cadre de cette recherche, le développement doit être appliqué de façon non-autoritaire par une rationalité argumentative scientifique. Cela permet de démontrer une apparence de neutralité par la maitrise des outils scientifique. Selon les personnes rencontrées, cette façon de faire dite « neutre » comporte trois conditions minimales, soit : avoir recours à une argumentation scientifique, laisser à l'interlocuteur le choix de retenir ou non la recommandation émise et, en dernier lieu, s'assurer de respecter les personnes, le projet expertisé, la culture, les institutions et les communautés qui reçoivent l'avis en adoptant un ton neutre dénué de toute opinion. 
Afin de démontrer une neutralité, l'argumentation des rapports et des recommandations doit être basée sur une argumentation ayant recours à la méthode et aux discours scientifiques, qui est acquise notamment via la formation académique. De plus, l'expert doit donner le choix à son interlocuteur de retenir ou non les recommandations émises. À terme, l'expertise se doit de présenter une argumentation rigoureuse sur la matière à évaluation dans l'optique où ce sera le seul objet de décision, car cette neutralité se doit en principe de structurer toute action de l'expert. L'utilisation d'outils scientifiques garantirait ainsi que les décisions et les recommandations ne sont pas imposées sur la base d'une opinion, mais plutôt sur la base d'un processus rigoureux se décrivant comme universellement objectif par rapport aux parties concernées.

L'habitus valorisé par le champ de l'expertise met ainsi en jeu deux processus de socialisation que les agents doivent avoir accompli pour avoir minimalement accès au champ et devenir des experts en développement international. Or, posséder les qualités requises ne fait pas d'un agent un expert en tant que tel, encore doit-il connaître les règles fondamentales et y naviguer tout en les respectant sans en révéler les contradictions.

\section{La doxa ou les présupposés du champ}

La doxa et les présupposés du champ constituent donc ces éléments qui présentent à la fois l'expertise comme vertueuse et masquent ses contradictions. Dans cette sous-section, nous exposerons la nature de la doxa dans ce champ, soit l'idéologie dont les principes structurent la composition du capital symbolique du champ de l'expertise et dans un second lieu l'illusio qui permet la domination des experts en matière de développement. 


\section{L'idéologie experte}

Pour être un bon expert en développement, comme nous l'avons vu dans la section des histoires d'horreur, l'expert doit, sous couvert d'un « amour profond des gens », travailler à prévenir les échecs de développement de manière impartiale dans le but mettre en valeur les actions de développement qui amélioreront la condition d'autrui selon les prédictions du modèle qu'il utilise. Derrière cette idéologie, on peut justifier l'existence du champ et mettre en lumière la règle qui en protège principalement la frontière conceptuelle. La sanction du développement : chasse gardée des experts.

L'entrée au champ suppose l'acceptation d'une valeur commune de tous les agents du champ. Maurice l'expose d'entrée de jeu lorsqu'il explique ce qu'est le développement:

Le développement international, il faut que ce soit traité comme une science. T'as des experts là-dedans, t'as un aspect économique qui est très important. Tu as des études à faire, tu as des recherches à faire, tu peux pas t'improviser comme ça [et dire :] « et bien moi, selon moi, on devrait faire ci, on devrait faire ça... » c'est fini [ce temps-là], c'est plus compliqué que ça [aujourd'hui].

En se décrivant comme expert, Maurice souligne d'entrée de jeu l'importance de la valeur commune dans la relation scientifique du développement et l'essence de cette relation. Le développement a besoin de l'expert et de son expertise. Sans l'expertise, le développement n'est rien. Il n'est que discours, qu'idéologie. Il devient impossible à confirmer. Dans cette optique, sans experts ni expertise, il serait impossible de savoir objectivement si les conditions de vie des gens se sont améliorées suite à l'application d'un projet, d'un programme ou d'une politique de développement. On trouve dans cette définition les éléments du paradoxe classique exposé par la littérature postdéveloppementiste, à savoir que le développement possède une définition variable qui dépend de la manière dont l'individu ou le groupe qui la proclame se définit et se représente (Rist 2007). En se 
présentant comme essentiel au développement et seul apte à attester de son existence, l'expert se pose dans le champ comme condition sine qua non et seul agent légitime en position d'affirmer qu'il y a développement.

\section{L'illusio ou la défense et la critique du développement}

La doxa veut qu'en respectant les règles minimales du champ, l'apport de l'expert en développement permettrait d'éviter « les histoires d'horreur ». C'est ainsi que la présence de l'expert aide à bien évaluer des besoins et à recommander les outils organisationnels qui permettront à des organismes locaux de faire le pont avec les donateurs canadiens en pratiquant les comportements recherchés. Or, l'organisation du développement est cadrée et identifie un type de partenaire organisé de façon spécifique sans avoir nécessairement un lien avec la culture locale. L'expert est donc envoyé pour former et familiariser les gens avec le modèle occidental de l'ONG qui transformera les groupes locaux en partenaires acceptables pour les donateurs canadiens et la bureaucratie étatique. Carl précise ainsi l'importance de l'aide experte pour ces groupes:

L'ONG est un concept profondément occidental, souvent les problèmes rencontrés vont être d'ordre organisationnel et au niveau du financement. Quand on le consulte pour son expertise, l'expert identifie les lacunes organisationnelles et il recommande ce que d'autres organismes qui ont vécu un problème similaire ont fait précédemment. Au niveau du financement, il peut mettre en contact avec des gens qui « savent comment le faire ».

Ainsi un expert solutionneur ou intellectuel apporte une critique des organismes en place, sur un autre axe, un traducteur-facilitateur ou une personne-ressource les accompagne dans leurs transformations vers un modèle d'organisation à l'occidentale pour leur permettre de trouver les subventions et de répondre aux critiques. En dernier lieu, tous ces types d'experts évaluent ensuite les réalisations de ces organismes tels des élèves en constante formation. L'expert doit ainsi défendre cette 
manière de procéder pour justifier son existence et se rendre indispensable.

Peu importe le type d'expertise qu'il estime pratiquer, l'expert est ainsi voué à défendre et à critiquer le développement dans une structure dont la doxa le contraint à se poser comme seul agent qualifié à émettre ou à sanctionner une critique du développement. L'expert se présente alors comme impartial dans l'optique où ses intentions sont nobles et qu'il est le seul à posséder la maîtrise des outils scientifiques lui permettant cette impartialité. Qui plus est, l'accès au champ est lui-même restreint car il valorise des habitus où les parcours académiques occidentaux et les nombreuses expériences de vie interculturelles en dehors de l'occident occupent une place prépondérante.

\section{V.4 - Résultats de l'analyse}

L'analyse des données recueillies dans le cadre de cette recherche nous indique que pour devenir expert, il faut être en mesure d'accumuler, de mobiliser, d'échanger ou de transformer des capitaux technologiques, organisationnels, sociaux et d'expertise. Ces capitaux sont ensuite utilisés par les experts pour attirer d'autres capitaux dans leur propre champ et maintenir leur statut. Le champ de l'expertise possède ainsi ses propres frontières et interagit principalement avec les agents du champ du pouvoir, du champ bureaucratique et du champ des expatriés. Or, pour entrer dans le champ, les experts doivent payer un droit d'entrée, soit réunir un parcours académique et des expériences de vie à l'étranger. Pour maintenir son expertise, et sa légitimité en tant qu'expert, l'expert se doit de pratiquer ces activités en présentant ses actions comme étant basées avant tout sur l'amour de l'autre et la nécessité d'être impartial et objectif dans la promotion du développement. Dans les faits, les experts sont appelés à jouer avec les limites de ces principes dans le but de pouvoir ramener assez de capital pour assurer leur survie et celle de l'organisation pour laquelle ils travaillent dans le champ de 
l'expertise en développement international. Pour ce faire, les experts sont placés dans un exercice d'équilibre périlleux. Ils sont chargés de critiquer le développement tel qu'il est pratiqué et de défendre sa dépendance au champ du pouvoir. Pour devenir experts, les agents doivent donc jouer le jeu sans quoi ils peuvent se voir exclus du champ. 


\section{CONCLUSION}

Nous avons commencé cette recherche en nous demandant « comment on devient expert en développement international ». Pour ce faire, nous avons mobilisé la théorie du champ de Pierre Bourdieu. Cette théorie propose de considérer les experts en développement international comme un espace social distinct centré autour de l'activité d'expertise qui possède ses propres logiques de domination et dont les individus et les groupes qui le composent détiennent leur propre habitus particulier. Ainsi, nous avons identifié que les experts pratiquent l'expertise en développement international principalement dans un cadre évaluatif des progrès accomplis par les populations et les groupes ciblés par le développement. Nous avons appris, suite à nos entrevues semi-dirigées, qu'une fois dans les communautés ciblées, les experts s'installent pour vivre dans un continuum qui selon la manière dont ils perçoivent leur rôle, va d'une proximité importante avec la communauté visée par le développement vers un second extrême où, à l'extérieur des communautés visées, les experts vivent dans la bulle culturelle de la communauté occidentale expatriée. Ce choix de lieu de vie a d'ailleurs une incidence marquée sur la manière dont l'expert va pratiquer son expertise et sur les habitus qu'il valorise comme par exemple la fréquentation de lieux particuliers. En dernier lieu, nous avons démontré que celui-ci base sa légitimité en se présentant comme un humaniste qui contribue au mieuxêtre des communautés grâce à son objectivité. Or, l'importance de l'influence du champ du pouvoir le place en réalité dans un exercice d'équilibre où il est appelé à critiquer le développement tout en se portant à la défense de sa dépendance au fonds de l'État canadien et de son champ du pouvoir, ce que l'oblige à négocier cette objectivité sur laquelle il base son autorité. Pour cette raison on notera qu'on 
fait ultimement appel à l'expert principalement dans les moments où il s'agit de déterminer s'il faut appuyer, poursuivre ou évaluer, une action de développement défendue par le champ du pouvoir canadien. Dans ce contexte, les personnes souhaitant devenir expertes en développement international doivent se munir d'un parcours académique universitaire et de nombreuses expériences de vie à l'étranger. Certains y réussissent mieux que d'autres et développent des habitus particuliers qui leur permettent de tirer profit de leur position dans le champ de l'expertise. Cela permet alors au champ de produire une multitude d'experts aux styles particuliers et uniques d'un individu à l'autre.

\section{Le champ de l'expertise : changement de régime et enjeux de lutte}

Si nous avons maintenant une idée de comment on devient expert, le sens commun nous induit à définir ce qu'est un expert en développement international. C'est pourquoi nous l'avons directement demandé aux personnes rencontrées dans le cadre de ce mémoire. Tous les répondants ont tout d'abord accepté de nous rencontrer pour parler d'expertise en développement international. Lorsque nous leur avons demandé « Êtes-vous un expert? », les réponses obtenues ont été variées : du « Oui! » au «Pas du tout» catégoriques que nous attendions se sont substituées des réponses plus hésitante: «Non, mais... » « Oui et non... », « Oui, je me présente comme un expert, mais je préfère le terme...». C'est à partir de ces hésitations que le cadre bourdieusien nous est apparu comme le plus à même de proposer une réponse à notre question à la fois ancrée dans la réalité objective et subjective des sujets de notre recherche. En effet, comme on le rappelait dans la présentation dudit cadre, c'est précisément la définition de ce qu'est un expert en développement international qui est l'enjeu dans ce champ qui subit l'influence de multiples champs comme le champ politique cherchant à tirer profit de l'aura de légitimité objective qui y est produite. De plus, au moment où nous avons fait nos observations, le champ était profondément perturbé par la désorganisation du champ bureaucratique de l'aide au 
développement de l'État canadien provoquée par les actions du gouvernement conservateur de l'époque. La théorie des champs de Bourdieu nous enseigne que c'est précisément dans ces moments que l'on peut discerner les tensions entre dominants et dominés. L'examen de la structure du champ à travers l'analyse de nos données met en lumière les éléments de domination observables : d'abord au niveau interne dans la nature des tensions observables dans le champ et ensuite au niveau externe, dans la nature des pressions qui sont imposées au champ de l'expertise en développement international et sur ses agents.

\section{Du dévoilement de la domination}

Au cours des entretiens menés dans le cadre de cette recherche, certaines interventions clés nous permettent d'affirmer qu'il existe des tensions vives entre l'expertise en action et le champ de l'expertise lui-même. Ces tensions se manifestent d'abord à l'interne, dans la manière de se présenter que certains experts préfèrent.

Certains revendiquent une appellation différente pour préserver l'intégrité de leur point de vue sur la manière de pratiquer l'expertise. Un peu comme les écrivains rencontrés par Bourdieu qui eux préféraient le terme « artiste » pour poser une critique de la relation marchande à la littérature, plusieurs des personnes rencontrées (Marie, Audrey, Gérald et Leonard) préfèrent rejeter le terme expert pour se définir par un terme comme celui de « spécialiste » et pour adopter un regard « critique » de l'expertise tel que le présente Leonard:

Je ne me suis jamais présenté ou qualifié comme un expert parce que ça me parait prétentieux et arrogant. Je préfère dire que j'ai de l'expérience dans certains domaines. Vous savez, j'ai travaillé dans plusieurs pays dans un domaine particulier, sur le même genre de dossiers. Vous voyez, je peux identifier le bon du mauvais, retenir les bons éléments et écarter les mauvais. 
Par cet exercice, Leonard pose une critique de la relation marchande à l'expertise que certains experts ont pu adopter et qui ternit la réputation de l'expertise en développement international aux yeux d'une part importante d'experts rencontrés. Il n'en demeure pas moins que Leonard poursuit des activités de développement et qu'il doit répondre aux critères de base pour avoir accès au champ de l'expertise en développement international.

En effet, la structure du champ de l'expertise donne lieu à ce genre de situations paradoxales. En dépit de son expertise, son cumul d'expériences et de capitaux, l'expert en développement international peut se retrouver soit dans une situation de rupture totale avec son champ, soit, dans une situation de dissonance avec son champ ou encore dans une situation où il est "pris au jeu" de son champ.

Si ces situations sont possibles, c'est que, dans bien des cas, l'idée que l'expert se fait de son devoir, soit son degré d'intégration de la doxa, est souvent bien plus important que les convictions personnelles intimes. C'est-à-dire que s'il souhaite survivre dans le champ de l'expertise en développement international, il se doit d'accorder plus d'importance aux valeur et règles de ce dernier qu'à ses propres valeurs. Pour cette raison, le degré d'importance que l'expert accorde à la doxa et son niveau de conscience des contradictions inhérentes aux règles qui l'accompagnent nous indiquent la nature des tensions qu'il expérimente dans l'action en développement international.

Ainsi la plupart des personnes rencontrées dans le cadre de cette recherche ne se sentent pas expertes ou ne peuvent pas être un expert parce qu'elles ne peuvent pas pratiquer leur expertise de façon autonome et impartiale. Les experts ne peuvent pas aborder certains éléments qu'ils estiment pertinents dans leurs rapports. Dans les cadres de travail, il n'y a pas d'espace pour expliquer les nuances, pour critiquer des politiques ou pour critiquer un programme hors des paramètres établis par 
le champ bureaucratique. Si les experts se retrouvent dans une telle situation, c'est que pour avoir un capital d'expertise suffisant, ils se doivent de compromettre leur expertise en abandonnant tout exercice critique non demandé. Ce qui pousse à ce comportement, c'est la logique comptable et la logique clientéliste dans laquelle sont imbriqués les organismes qui ont recours à leur travail, une logique qui transcende les différents secteurs d'expertise ${ }^{22}$. L'expert se trouve alors pris au jeu du champ de l'expertise. Il est alors soit conscient d'être pris à ce jeu et il accepte cette situation avec fatalité, soit conscient d'être pris au jeu sans savoir quoi faire pour s'en déprendre autrement qu'en quittant le champ où il est complètement aliéné.

Nous croyons que l'expert pris au jeu qui ne semble pas avoir de solution est dans cette situation pour deux raisons. Premièrement, le champ est perturbé par l'influence du champ du pouvoir qui influence la valeur de certains capitaux ce qui limite graduellement l'autonomie du champ, donc l'impartialité des experts, soit leur capital principal. Deuxièmement, le champ est aussi perturbé par la valeur sans cesse grandissante accordée aux capitaux en termes d'unités de temps qu'entrainent les différentes exigences du champ du pouvoir en échange du capital économique qu'il fournit. L'expert est de plus en plus limité en raison de l'accélération croissante du rythme de péremption des capitaux technique et social. En même temps, on demande aux projets de développement de délivrer sans cesse des résultats plus grands et plus probants, malgré une unité de temps qui reste la même, et une temporalité des communautés ciblées qui n'évolue pas au même rythme.

L'expert se trouve dans les situations décrites dans les résultats, car il ne possède pas suffisamment de ces capitaux, et se trouve alors toujours pressé par le temps pour répondre aux

22 La liste des champs d'expertise qui subissent les forces contraignantes asservissant les expertises est longue dans la littérature scientifique. On y trouve une littérature variée à propos de son impact sur des professions hétéroclites telles que les travailleurs sociaux (Blanc 2009), les pédagogues (Hursh 2000), les chercheurs en environnement (Lave 2012), les médecins vétérinaires (Enticott et al. 2011) ou encore même les joueurs de Rugby (Smith 2000)(!) 
exigences des acteurs issus du champ du pouvoir, « acheteurs » quasi exclusifs des capitaux produits par le champ de l'expertise. Les experts se trouvent alors en tension pour critiquer et défendre ce qui contraint leur action. Dans certains cas, cela provoque un sentiment d'impuissance malgré la compétence des experts, car ces derniers possèdent les outils pour critiquer à la fois à l'intérieur du cadre qu'on leur impose et pour critiquer le cadre lui-même sans pour autant avoir une emprise sur ce dernier. Dans un tel cas, le champ de l'expertise canadienne en développement international devient excessivement dépendant du groupe qui détient le pouvoir et des critères idéologiques qu'il invoque pour justifier son autorité.

Or, les interventions des divers agents tendent à démontrer que les agents rencontrés sont en réaction face à une perturbation extérieure du champ. Sans le dire explicitement, le commentaire de Carl que nous relevions à propos de l'ambiance dans le champ du développement prend tout son sens; il résume en quelques lignes comment le changement des critères d'attribution des fonds au développement provoque une crise profonde dans le champ de l'expertise, directement sous l'influence du champ bureaucratique de l'ACDI et de l'État canadien:

L'ACDI, en voulant devenir plus efficace et pour réduire ses coûts, s'était mis à reconnaître des organismes comme compétents et à leur attribuer des enveloppes de financement, ce qui donnait plus de liberté aux ONG pour gérer ces fonds. On avait donc des gouvernements qui avaient confiance dans les ONG, un climat différent du climat actuel. Alors aujourd'hui ces organisations doivent toutes faire des soumissions et quand on décide qui va recevoir, il n'y a pas de transparence dans le processus, on ne dit pas au rejeté pourquoi ils ne se qualifient pas pour du financement, d'autres perdent leur financement pour des raisons politiques tel que CAIROS ou encore Développement et paix. Pour ces raisons il y a un froid actuellement dans la communauté du développement canadien.

Des règles du champ du développement qui protégeaient certains types d'activités ont changées pour exclure des connaissances et des positions politiques portées par certaines ONG au profit d'autres agents qui portent les valeurs du champ politique. Cela a un impact direct sur le champ de l'expertise, 
car la valeur de certains capitaux diminue alors que celle d'autres capitaux augmente ce qui change les conditions d'accès au champ. Cette transformation de l'environnement exclut dès lors certains agents et facilite l'entrée d'autres aux habitus auparavant étrangers au champ. Elle nous indique toute l'importance de l'influence que le champ du pouvoir et l'incertitude dans le champ bureaucratique peuvent avoir sur le champ de l'expertise canadienne en développement international. Les changements de régime politique ont donc une influence considérable sur la hiérarchisation des luttes du champ.

\section{Les changements de régime au Canada}

Le champ de l'expertise en développement international occupe une place particulière dans le champ du développement. Un peu comme dans une variante en trois dimensions d'un jeu d'échecs (Eisenberg 2007), nos résultats et la mise en perspective avec le contexte politique dans lesquels ils ont été obtenus nous indiquent que le champ de l'expertise canadienne en développement international subit l'influence de nombreux autres champs et plus particulièrement celle du champ du pouvoir canadien par le truchement du champ bureaucratique de l'État canadien. Indirectement, les champs qui ont une influence importante sur le champ du pouvoir structurent la nature des relations du champ de l'expertise avec l'extérieur ainsi que la nature des luttes qui y ont cours. Dans la période où nous avons rencontré ces personnes soit au cours de la seconde moitié de l'année 2013, l'actualité du champ était caractérisée par l'influence écrasante exercée par les entreprises extractives et les organisations religieuses protestantes sur le champ politique canadien à travers le parti conservateur de Stephen Harper aux commandes de l'État canadien (Morin et Roussel 2014, 6; Paquette 2014). De nombreux gestes visant à limiter l'autonomie des organismes de développement international ont été posés dans les années précédentes et les budgets alloués à l'aide au développement diminuent sans cesse. Mais en mars 2013, alors au milieu de son mandat, le gouvernement conservateur de Stephen Harper ordonne la 
fusion de l'ACDI avec le MAECD ce qui provoque une inquiétude galopante et de profondes remises en question dans le milieu des ONG de développement international (Beaudet 2013; Leclerc 2013).

Dans un tel climat, le champ de l'expertise se trouve en lutte pour sauvegarder son autonomie visà-vis des champs qui dominent notre société, notamment le champ économique et le champ du pouvoir. Or l'influence, la puissance grandissante de ces derniers et la matérialisation de celle-ci dans le champ de l'expertise en développement international provoquent, à notre avis, une crise au sein du champ. En effet, les tensions sont perceptibles dans l'attitude et les discours des experts rencontrés qui tous sans exception sentent leur indépendance menacée d'une façon ou d'une autre par ce qui est perçu par plusieurs comme la perte de l'indépendance scientifique et morale de l'aide canadienne au développement et plus précisément comme un abandon de sa mission de réduction de la pauvreté. Elles le sont au point où Leonard nous annonce en fin d'entretien son intention de s'impliquer dans le champ politique dans le but d'aller expressément à la défense de l'indépendance du champ de l'expertise et de son caractère scientifique. Si l'on fait un saut dans le temps suite aux entretiens, la campagne électorale de 2015 voit les libéraux de Justin Trudeau faire d'un retour à « aider les plus pauvres » dans un cadre « guidé par les faits et les résultats » un point important de leur plateforme politique en matière de politique étrangère (Parti libéral du Canada 2015). Toutefois, les réactions aux annonces en la matière du nouveau gouvernement libéral en lien avec leur budget 2016 ont étés teintées de prudence chez les représentants des ONG (AQOCI 2016). En effet, ces derniers attendent essentiellement de voir si leur relative autonomie leur sera restituée avant de se réjouir. Ce changement de régime politique dans la capitale canadienne laisse donc ouvertes plusieurs possibilités quant à l'évolution de la situation du champ de l'expertise en développement international et limite donc la portée des conclusions de cette recherche. 


\section{Limites de la recherche}

En effet, on comprendra que le moment choisi pour rencontrer des experts aura teinté lourdement les données produites dans le cadre de cette recherche. Si on tient compte de l'effet de ce contexte, il aura limité la propension de certains acteurs à s'ouvrir à nous et à se sentir en sécurité en participant à notre étude, notaient les experts à l'emploi d'une agence gouvernementale sur le point de disparaître. De plus, nos demandes de rencontres auront incité surtout ceux qui voulaient s'exprimer et qui se sentaient assez en sécurité dans le champ pour traiter du sujet de l'expertise en cette époque marquée par la fin annoncée de l'ACDI et les coupures budgétaires massives.

Par ailleurs, le changement de régime politique à Ottawa limite les portées éventuelles des apprentissages de cette recherche. En effet, il y a fort à parier que nombre des éléments mis en lumière ont perdu de leur utilité en raison du temps nécessaire à la rédaction et à la publication de nos résultats. L'unité dans les discours et les arguments lancés à l'encontre du gouvernement Harper ou de ses actions a limité les occasions d'observer les différences de positions internes du champ. Encore une fois, le contexte a favorisé la mise en lumière d'un champ en réaction face à ce qui est unanimement perçu par ses agents comme une ingérence du champ du pouvoir. Cependant, elles nous auront permis de voir à quel point le champ peut trouver une forme d'unité autour de la défense de son indépendance et ainsi de contribuer à rendre plus apparents et facilement observables l'illusio, la doxa et le prix d'entrée du champ au détriment de ses tensions internes.

Sur le plan, méthodologique, le petit nombre de personnes rencontrées, la proportion importante d'individus âgés et la surreprésentation des hommes dans l'échantillon auront eu pour effet de limiter la différenciation des expériences rapportées. En effet, nous avons exclu la perspective du genre de notre 
cadre d'analyse. La recherche produite est donc aveugle face aux spécificités du devenir expert des femmes et des minorités visibles ou sexuelles. Nous nous permettons cependant de souligner l'importance dans d'éventuelles recherches futures de s'attarder à ces aspects de la problématique que nous n'avons pas abordés pour mieux comprendre la structure et la construction des habitus dans un champ où la question de genre occupe une place importante dans les connaissances valorisées.

D'un autre côté, la présence importante de personnes plus expérimentées ou récemment retraitées aura permis de solidifier l'aspect historique de notre recherche et de valider la continuité dans le temps jusqu'à la période observée de la valorisation d'un certain type d'habitus expert et de la stabilité du prix d'entrée dans le champ. Par contre, cela ne nous permet pas de nous prononcer avec conviction sur la stabilité future de ces derniers suite à l'ampleur des perturbations subies par le champ. En effet, faire une enquête sur le présent de l'expertise est problématique parce que le passé continue de se réfléchir sur le présent dans la manière dont les gens abordent les défis rencontrés.

Pour terminer, la richesse des discours produits nous aura permis d'identifier les éléments principaux de la structure du champ de l'expertise en développement international et de répondre adéquatement à notre question de recherche. De plus, cette recherche aura été riche en expérience pour le chercheur et la suite de ses projets.

\section{Retour sur les expériences personnelles du chercheur}

Sur le plan personnel, cette recherche nous aura amené beaucoup. D'une part, elle nous aura permis de préparer notre première expérience en termes de solidarité internationale. Dans le cadre de cette recherche, nous avons rencontré des personnes extraordinaires, riches en expériences diverses, 
ouvertes, excessivement généreuses de leur temps et de leurs enseignements. Cela nous a grandement préparé à apprivoiser le «terrain » de la solidarité internationale. En effet, d'avril 2014 à juin 2014, nous avons effectué un séjour de dix semaines dans la petite communauté de Tandahota au Bénin. Ces rencontres nous ont permis de nous trouver des repères plus facilement dans un environnement qui nous était totalement étranger et d'appréhender les défis que nous aurions à relever. Elles nous ont aussi permis de comprendre l'importance d'être à proximité des communautés avec qui l'on doit travailler, d'apprendre à douter de ses premières impressions et surtout d'être patient pour récolter le fruit de la rencontre de l'autre.

Sur le plan professionnel, nous éprouvions, au début, un intérêt fort à travailler dans ce milieu, à devenir nous-même « expert en développement international ». Malgré le lot de désillusion qu'elle nous a apporté, cette recherche nous a permis de mieux saisir les enjeux qui nous dépassent en tant que personne et d'observer les différentes stratégies et attitudes mises en œuvre par les experts pour naviguer dans un champ agité. Cette recherche aura renforcé notre conviction que nous sommes à même de participer avec ces gens à l'avènement d'un monde meilleur. Par ailleurs, elle aura su nous outiller et nous donner des informations importantes qui seront plus qu'utiles dans le cadre de nos propres recherches d'emploi en développement. En effet, mieux comprendre le contexte et la structure dans laquelle naviguent ces personnes remarquables nous amène à souligner leur courage face à l'adversité. La patience qu'ont ces derniers pour attendre les moments opportuns où saisir les occasions de défendre leurs convictions les plus profondes ou encore pour les provoquer mérite qu'on souligne leurs compétences et qu'on en tire les enseignements qui s'imposent.

Sur le plan de l'expérience scientifique, cette recherche nous pousse à vouloir continuer plus loin dans l'examen de la manière dont on devient expert en développement international. En effet, étant au 
tout début d'une carrière dans le milieu, nous croyons qu'il serait des plus intéressants de poursuivre ce questionnement et de procéder à une autoethnographie du processus dans le cadre d'une future recherche doctorale. La singularité des expériences rencontrées nous pousse à mieux vouloir comprendre notre propre expérience individuelle du développement international et de notre transformation en expert. 


\section{BIBLIOGRAPHIE}

Agence canadienne de développement international. 2002. Canada making a difference in the world: a policy statement on strengthening aid effectiveness. Hull, Quebec: Canadian International Development Agency.

Akrich, Madeleine, Michel Callon et Bruno Latour. 2006. Sociologie de la traduction: textes fondateurs. Presses des MINES.

Andersen, Regine. 2000. «How multilateral development assistance triggered the conflict in Rwanda ». Third World Quarterly, vol. 21, no 3, p. 441-456.

Anderson, Mary B, Dayna Brown et Jean Isabella. 2012. Time to listen: hearing people on the receiving end of international aid. Cambridge, MA : CDA Collaborative Learning Projects. En ligne. $<\underline{\text { http://www.cdacollaborative.org/media/60478/Time-to-Listen-Book.pdf }>\text {. }}$

Association québécoise des organismes de coopération internationale. 2016. « Budget 2016-2017 : Les libéraux ont-ils respecté leurs engagements pour l'aide internationale ? - Association québécoise des organismes de coopération internationale (AQOCI) ». En ligne. <http://www.aqoci.qc.ca/? Budget-2016-2017-Les-liberaux-ont>. Consulté le 26 juin 2016.

Atlani-Duault, Laëtitia. 2006. « Entre Mémoire et oubli: la construction institutionnelle de la souffrance sociale. Un essaie d'anthropologie politique de l'aide au développement ». Dans Paroles d'experts: études sur la pensée institutionnelle du développement., sous la dir. de Jean-François Baré, p. 239-288. Paris : Karthala-L'Harmattan.

Banerjee, Abhijit V. et Esther Duflo. 2011. Poor economics: a radical rethinking of the way to fight global poverty. 1 st ed. New York : PublicAffairs.

Barbier, Marc, Lionel Cauchard, Pierre-Benoít Joly, Catherine Paradeise et Dominique Vinck. 2013. «Pour une Approche pragmatique, écologique et politique de l'expertise ». Revue d'anthropologie des connaissances, vol. 7, no 1, p. 1-23.

Barnett, Michael N. et Martha Finnemore. 2003. « The Politics, Power, and Pathologies of International Organizations ». International Organization, vol. 53, no 04, p. 699-732.

Barthes, Roland. 1957. «Le mythe aujourd'hui ». Dans Mythologies, p. 191-247. Paris, France : Éditions du Seuil.

Beaudet, Pierre. 2013. "La fin de l'ACDI, prévue et prévisible ». Le Devoir, 23 mars 2013, sect. Politique. En ligne. $<\underline{\text { http://www.ledevoir.com/politique/canada/373964/la-fin-de-l-acdi-prevue- }}$ et-previsible>.

Becker, Gary S et Kevin M Murphy. 1988. « A theory of rational addiction ». The journal of political economy, p. 675-700. 
Becker, H.S. 1956. «Interviewing medical students». American Journal of Sociology, no 62, p. 199-201.

Berque, Jacques. 1965. « Pour une sociologie de l'assistance technique ». Dans De l'impérialisme à la décolonisation, par Gabriel Ardant, Jacques Berque, et Jean-Paul Charnay, p. 433-444. Paris : Les Éditions de Minuit.

Berrebi-Hoffmann, Isabelle et Michel Lallement. 2009. « À quoi servent les experts ? Cahiers internationaux de sociologie, no 126, p. 5-12.

Bhabha, Homi K. 1994. The location of culture. Psychology Press.

Bhushan, Aniket. 2013. Beyond Aid: Trade, Investment, and Remittances between Canada and Developing Countries. Coll. «Research Report». The North South-Institute. En ligne. $<$ http://cidpnsi.ca/wp-content/uploads/2012/11/Beyond-Aid-Trade-Investment-andRemittances-between-Canada-and-Developing-Countries-Bhushan2013.pdf $>$.

Black, David. 2009. « Out of Africa? The Harper government's new "tilt" in the developing world ». Canadian Foreign Policy Journal, vol. 15, no 2, p. 41-56.

Blanchet, Alain. 1982. «Épistémologie critique de l'entretien d'enquête de style non directif ; ses éventuelles distorsions dans le champ des sciences humaines ». Bulletin de Psychologie, no 358, p. 187-195.

—. 2005. «Interviewer». Dans Les Techniques d'enquête en sciences sociales: observer, interviewer, questionner, p. 81-126. Paris : Dunod.

Blanc, Maurice. 2009. «Le renouvellement démocratique des pratiques sociales: Enjeux politiques et défis pratiques pour le travail social dans la ville et le quartier ». Nouvelles pratiques sociales, vol. 22, no 1, p. 13.

Bøås, Morten et Desmond McNeill. 2003. « Introduction: Power and Ideas in Multilateral Institutions: Towards an Interpretative Framework ». Dans Global institutions and development: framing the world?, sous la dir. de Morten Bøås et Desmond McNeill. Routledge.

Boas, Taylor C. et Jordan Gans-Morse. 2009. « Neoliberalism: From New Liberal Philosophy to AntiLiberal Slogan ». Studies in Comparative International Development, vol. 44, no 2, p. 137-161.

Boltanski, Luc. 2005. «Usages faibles, usages forts de l'habitus ». Dans Travailler avec Bourdieu, sous la dir. de Pierre Encrevé et Rose-Marie Lagrave, p. 153-161. Paris, France : Flammarion.

—. 2009. De la critique: précis de sociologie de l'émancipation. Coll. « NRF Essais ». Paris: Gallimard.

Boltanski, Luc et Eve Chiapello. 1999. Le nouvel esprit du capitalisme. Gallimard.

Bourdieu, Pierre. 1958. Sociologie de l'Algérie. $1^{\text {re }}$ éd. Paris : PUF. 
. 1979a. «Les trois états du capital culturel». Actes de la recherche en sciences sociales, vol. 30, no 1, p. 3-6.

1979b. La distinction: critique sociale du jugement. Coll. « Le Sens commun». Paris : Éditions de Minuit.

—. 1980a. Le sens pratique. Coll. « Le Sens commun ». Paris : Éditions de Minuit.

—. 1980b. « Le capital social ». Actes de la recherche en sciences sociales, p. 2-3.

—. 1981. «La représentation politique: Éléments pour une théorie du champ politique ». Actes de la recherche en sciences sociales, vol. 36, no 1, p. 3-24.

—. 1984a. Homo academicus. Coll. «Collection «Le Sens commun»». Paris : Editions de Minuit.

- 1984b. «La délégation et le fétichisme politique ». Actes de la recherche en sciences sociales, vol. 52, no 1, p. 49-55.

—. 1986. «La force du droit: Eléments pour une sociologie du champ juridique ». Actes de la recherche en sciences sociales, vol. 64, no 1, p. 3-19.

—_. 1991. « Le champ littéraire ». Actes de la recherche en sciences sociales, vol. 89, no 1, p. 3-46.

. 1992. Les règles de l'art: genèse et structure du champ littéraire. Coll. «Libre examen. Politique ». Paris : Editions du Seuil.

—. 1993a. « Esprits d'Etat: Genèse et structure du champ bureaucratique ». Actes de la recherche en sciences sociales, vol. 96, no 1, p. 49-62.

_. 1993b. La misère du monde. [Paris] : Éd. du Seuil.

—. 1994. Raisons pratiques: sur la théorie de l'action. Paris : Seuil.

. 1997a. «Le champ économique ». Actes de la recherche en sciences sociales, vol. 119, no 1, p. 48-66.

—. 1997b. Méditations pascaliennes. Coll. « Collection Liber ». Paris : Seuil.

—. 2000. Propos sur le champ politique. Lyon : Presses universitaires de Lyon.

-2002. Questions de sociologie. Paris : Ed. de Minuit.

- 2011. «Champ du pouvoir et division du travail de domination: Texte manuscrit inédit ayant servi de support de cours au Collège de France, 1985-1986 ». Actes de la recherche en sciences sociales, vol. 190, no 5, p. 126. 
- 2013. «Séminaires sur le concept de champ, 1972-1975: Introduction de Patrick Champagne ». Actes de la recherche en sciences sociales, vol. 200, no 5, p. 4.

Bourdieu, Pierre, Jean-Claude Chamboredon et Jean-Claude Passeron. 1968. Le métier de sociologue: préalables épistémologiques. Sous la dir. de. Beate Krais. Coll. "Textes sociologiques». Berlin : Mouton de Gruyter.

Bourdieu, Pierre et Yvette Delsaut. 1975. « Le couturier et sa griffe : contribution à une théorie de la magie ». Actes de la recherche en sciences sociales, vol. 1, no 1, p. 7-36.

Bourdieu, Pierre et Monique de Saint Martin. 1978. « Le patronat ». Actes de la recherche en sciences sociales, vol. 20, no 1, p. 3-82.

Bourdieu, Pierre et Jean-Claude Passeron. 1964. Les héritiers: les étudiants et la culture. Coll. «Le sens commun ». Paris : Les Éd. de Minuit.

1970. La reproduction: éléments pour une théorie du système d'enseignement. Coll. « Le Sens commun ». Paris : Éd. de Minuit.

Bourdieu, Pierre et Abdelmalek Sayad. 1964. Le déracinement: la crise de l'agriculture traditionnelle en Algérie. $2^{\mathrm{e}}$ éd. Paris : Les Éd. de Minuit.

Bourdieu, Pierre et Loíc J. D. Wacquant. 1992. Réponses: pour une anthropologie réflexive. Coll. « Libre examen Politique ». Paris : Ed. du Seuil.

Bourdieu, Pierre et Loïc J. D. Wacquant. 1998. « Sur les ruses de la raison impérialiste ». Actes de la recherche en sciences sociales, vol. 121, no 1, p. 109-118.

Bourguignon, François. 2012. «Le pouvoir des organisations internationales sur le développement: illusion ou réalité? » Tracés, no 3, p. 247-265.

Brender, Natalie. 2012. «CSR Funding is the Least of CIDA's Woes». CIPS. En ligne.

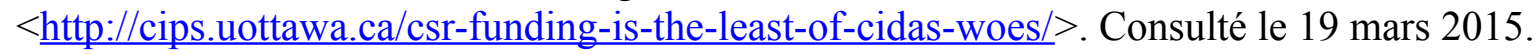

Brenner, Neil, Jamie Peck et Nik Theodore. 2010. «Variegated neoliberalization: geographies, modalities, pathways ». Global Networks, vol. 10, no 2, p. 182-222.

Brenner, Neil et Nik Theodore. 2002. "Cities and the geographies of "actually existing neoliberalism" ». Antipode, vol. 34, no 3, p. 349-379.

Brint, Steven. 1996. In an age of experts: The changing role of professionals in politics and public life. Princeton University Press.

Broad, Robin. 2006. « Research, knowledge, and the art of 'paradigm maintenance': the World Bank's Development Economics Vice-Presidency (DEC) ». Review of International Political Economy, vol. 13, no 3, p. 387-419. 
- 2007a. «'Knowledge management': a case study of the World Bank's research department ». Development in Practice, vol. 17, no 4-5, p. 700-708.

Brown, Stephen. 2012. «Putting the Corporate Back Into CSR: A Rejoinder to Natalie Brender ». CIPS. En ligne. <http://cips.uottawa.ca/putting-the-corporate-back-into-csr-a-rejoinder-to-

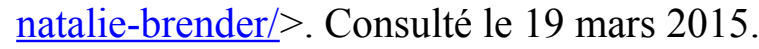

- 2013. «Canadian Aid to Africa ». Dans Canada-Africa relations: looking back, looking ahead, sous la dir. de Norman Paterson School of International Affairs et Centre for International Governance Innovation, par Rohinton Phiroze Medhora et Yiagadeesen Samy, p. 181-194. Coll. «Canada Among Nations ». Waterloo, ON; [Ottawa] : Centre for International Governance Innovation; Norman Paterson School of International Affairs.

Canada. 2013. Réponse du gouvernement au sixième rapport du comité permanent des affaires étrangères et du développement international. En ligne .

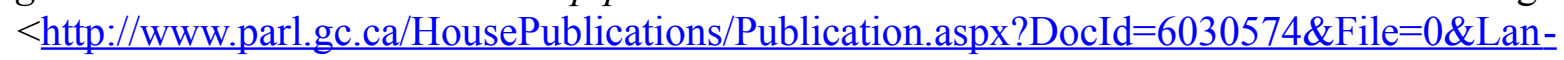
guage $=\mathrm{F}>$.

Canada, Ministère des finances et James M Flaherty. 2007. Le budget de 2007: viser un Canada plus fort, plus sécuritaire et meilleur : le nouveau Gouvernement du Canada. Ottawa : Ministère des finances. En ligne. <http://www.budget.gc.ca/2007/pdf/bp2007f.pdf>.

Carapico, Sheila. 2002. " Foreign aid for promoting democracy in the Arab world ». The Middle East Journal, p. 379-395.

Cassen, Robert. 1994. Does aid work?: report to an intergovernmental task force. Oxford; New York : Clarendon Press; Oxford University Press.

Césaire, Aimé. 2004. Discours sur le colonialisme. Paris : Présence Africaine.

Coleman, James S. 1988. «Social capital in the creation of human capital ». American journal of sociology, p. S95-S120.

Collier, Paul. 2006. «Is Aid Oil? An Analysis Of Whether Africa Can Absorb More Aid». World Development, vol. 34, no 9, p. 1482-1497.

Collier, Stephen J. 2012. « Neoliberalism as big Leviathan, or ... ? A response to Wacquant and Hilgers » Social Anthropology, vol. 20, no 2, p. 186-195.

Collins, Harry. M. et Robert Evans. 2002. «The Third Wave of Science Studies: Studies of Expertise and Experience ». Social Studies of Science, vol. 32, no 2, p. 235-296.

2008. Rethinking Expertise. Chicago : The University of Chicago Press. 
Conseil canadien pour la coopération internationale. 2001. «Propositions pour délier l'aide canadienne Note d'information politique du CCCI». Ressources. En ligne .

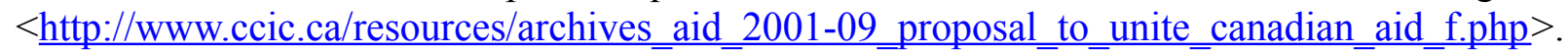
Consulté le 3 septembre 2014.

. 2014. Utiliser le secteur privé comme levier? Comprendre et analyser la collaboration des organisation canadiennes de développement internationl avec le secteur privé par le biais du plaidoyer, $d u$ dialogue de la promotion et des partenariats. En ligne. $<$ http://www.ccic.ca/ files/fr/what we do/2014 03 26 PSE Executive_Summary.pdf $>$. Consulté le 3 septembre 2014.

Cornwall, Andrea et Karen Brock. 2005. «What do buzzwords do for development policy? a critical look at 'participation', 'empowerment' and 'poverty reduction'». Third World Quarterly, vol. 26, no 7, p. 1043-1060.

Crozier, Michel. 1971. Le phénomène bureaucratique: essai sur les tendances bureaucratiques des systèmes d'organisation modernes et sur leurs relations en France avec le systèmes social et culturel. Paris : Ed. du Seuil.

Crozier, Michel et Friedberg, Erhard. 1977) L'acteur et le systéme: les contraintes de l'action collective. Paris : Éd. du Seuil.

Debord, Guy. 2006. La société du spectacle. Chicoutimi : J.-M. Tremblay. En ligne.

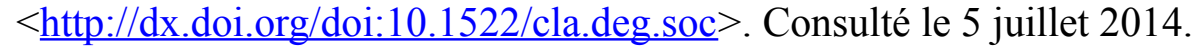

Della Faille, Dimitri. 2012. "Les études postcoloniales et le "sous-développement" . Revue québécoise de droit international, vol. Hors-série novembre 2012, p. 11-31.

Della Faille, Dimitri, Valérie La France-Moreau et Laurent Paradis-Charette. 2016. « Expertise et processus de prise de décision en développement international : proposition d'une typologie ». Politique et sociétés, vol. 35, no 2-3, p. 215-238.

Djankov, Simeon, Jose G. Montalvo et Marta Reynal-Querol. 2008. « The curse of aid ». Journal of Economic Growth, vol. 13, no 3, p. 169-194.

Doucouliagos, Hristos et Martin Paldam. 2009. « The Aid Effectiveness Literature: The Sad Results of 40 Years of Research ». Journal of Economic Surveys, vol. 23, no 3, p. 433-461.

Duménil, Gérard et Dominique Lévy. 2011. The crisis of neoliberalism. Harvard University Press.

Dumoulin, Laurence, Stéphane Labranche, Cécile Robert et Philippe Warin. 2005. Le recours aux experts. Raisons et usages politiques. Presses Universitaires de Grenoble. En ligne. $<\underline{\text { http://halshs.archives-ouvertes.fr/halshs-00290250/en/>. }}$.

Easterly, Professor William. 2013. The Tyranny of Experts. Washington : BASIC CIVITAS BOOKS. 
Easterly, William. 2006. The white man's burden: why the West's efforts to aid the rest have done so much ill and so little good. New York : Penguin Press.

Eisenberg, Anne F. 2007. « habitus/field». Sous la dir. de. George Ritzer. The Blackwell encyclopedia of sociology. Malden, MA : Blackwell Pub.

Elster, Jon. 1979. Ulysses and the sirens: Studies in rationality and irrationality. Vol. 1. Cambridge University Press Cambridge.

Engels, Friedrich et Karl Marx. 1974. L’idéologie allemande. Trad. par Joseph Weydemeyer, Guillaume Fondu, Jean Quétier, et Jean-Pierre Lefebvre. Ed. sociales.

Enticott, G., A. Donaldson, P. Lowe, M. Power, A. Proctor et K. Wilkinson. 2011. " The changing role of veterinary expertise in the food chain ». Philosophical Transactions of the Royal Society B: Biological Sciences, vol. 366, no 1573, p. 1955-1965.

Escobar, Arturo. 1995. Encountering development: The making and unmaking of the Third World. Princeton Univiversity Press.

Essex, Jamey. 2012. « The politics of effectiveness in Canada's international development assistance ». Canadian Journal of Development Studies/Revue canadienne d'études du développement, vol. 33, no 3, p. 338-355.

Evans, Robert. 2008. «The Sociology of Expertise : The Distribution of Social Fluency ». Sociology The Journal Of The British Sociological Association, vol. 1, no 2, p. 281-298.

Fanon, Frantz. 2002. Les damnés de la terre. 2e éd. Coll. «La découverte 134 ». Paris : La Découverte/poche.

Ferguson, James. 1994. The anti-politics machine: " development 》, depoliticization, and bureaucratic power in Lesotho. Minneapolis : University of Minnesota Press.

2006. Global shadows: Africa in the neoliberal world order. Duke University Press.

—. 2009. « The Uses of Neoliberalism ». Antipode, vol. 41, p. 166-184.

Foucault, Michel. 2004. Naissance de la Biopolitique Cours au Collège de France, 1978-1979. Seuil/Gallimard. Paris, France.

Freyss, Jean. 2004. « La solidarité internationale, une profession? » Revue Tiers Monde, vol. 4, no 180, p. 735-772.

Friedman, Georges. 1946. Problèmes humains du machinisme industriel. Coll. « Machine et humanisme ». Paris, France : Gallimard. 
Gaboury, Paul. 2015. « Abolition de postes dans la fonction publique: une facture de 4,45 milliards ». Le Droit, 23 janvier 2015. En ligne. <http:/www.lapresse.ca/le-droit/politique/fonctionpublique/201501/22/01-4837698-abolition-de-postes-dans-la-fonction-publique-une-facture-de445-milliards.php $>$. Consulté le 2 août 2016.

George, Susan et Fabrizio Sabelli. 1994. Crédit sans frontières; La religion séculière de la banque mondiale. Trad. par Christophe Delprat. La découverte. Paris.

Gilmore, Scott. 2014. "The Big Idea: Canada is dangerously disengaged with the world: expert» Entretien réalisé par Tom Clark.Global News. En ligne. $<\underline{\text { http://globalnews.ca/news/1351574/canada-is-dangerously-disengaged-with-the-world- }}$

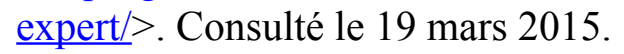

Gilroy, Paul. 1991. "There Ain't no Black in the Union Jack': the Cultural Politics of Race and Nation . Coll. « Black literature and culture ». Chicago, Ill : University of Chicago Press.

Gonthier, Frédéric. 2014 . «Habitus». Encyclopadia Universalis. E n $1 \mathrm{ig} \mathrm{ne}$.

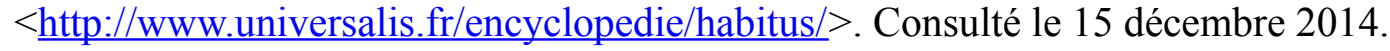

Gore, Charles. 2000. « The Rise and Fall of the Washington Consensus as a Paradigm for Developing Countries ». World development, vol. 28, no 5, p. 789-804.

Goulet, Denis. 1980. « Development Experts: The One-eyed Giants ». World Development, vol. 8, no $7-8$, p. $481-489$.

Grawitz, Madeleine. 2001. Méthodes des sciences sociales. Paris : Dalloz.

Green, Maia. 2012. « Framing and Escaping; Contrasting Aspects of Knowedge Work in International Development and Anthropology ». Dans Differentiating Development; beyond an Anthropology of Critique, sous la dir. de Soumhya Venkatesan et Thomas Yarrow, p. 42-57. Berghahn Books. Oxford.

Griffith, Peter. 2003. The Economist's Tale: A Consultant Encounters Hunger and the World Bank. Londres : Zed Books.

de Haan, Arjan. 2009. How the Aid Industry Works: An Introduction to International Development. Sterling, VA : Kumarian Press.

Hall, Stuart. 1993. «What is this“"Black” in Black Popular Culture? » Social Justice, p. 104-114.

Hancock, Graham. 1992. Lords of Poverty: The Power, Prestige, and Corruption of the International Aid Business. Atlantic Monthly Press.

Harman, Sophie et David Williams. 2014. « International Development in Transition ». International Affairs, vol. 90, no 4, p. 925-941. 
Hilgers, Mathieu. 2012. «The historicity of the neoliberal state ». Social Anthropology, vol. 20, no 1, p. 80-94.

Hilgers, Mathieu et Éric Mangez. 2014. «Introduction to Pierre Bourdieu's Theory of Social Fields" ». Dans Bourdieu's theory of social fields: concepts and applications, par Mathieu Hilgers et Éric Mangez, p. 1-36. Coll. « Routledge advances in sociology ». New York, NY : Routledge, Taylor \& Francis Group.

Hoffman, Lisa, Monica DeHart et Stephen J Collier. 2006. " Notes on the Anthropology of Neoliberalism ». Anthropology News, vol. 47, no 6, p. 9-10.

Hursh, David. 2000. « Neoliberalism and the Control of Teachers, Students, and Learning: The Rise of Standards, Standardization, and Accountability ». Cultural Logic, vol. 4, no 1.

ICI.Radio-Canada.ca, Zone Politique-. 2012. «Des scientifiques canadiens se disent muselés par Ottawa $) \quad$ Radio-Canada.ca. $\quad$ E n $\quad$ i g n e . canada.ca/nouvelles/Politique/2012/02/17/003-scientifiques-critiques-harper.shtml $>$. Consulté le 2 août 2016.

Jessop, Bob. 2002. «Liberalism, Neo-Liberalism and Urban Governance: A State Theoretical Perspective. » Antipode, vol. 34, no 3, p. 452-472.

Kahneman, Daniel. 2003. « Maps of Bounded Rationality: Psychology for Behavioral Economics ${ }^{\dagger} »$. American Economic Review, vol. 93, no 5, p. 1449-1475.

Kennedy, David. 2005. « Challenging Expert Rule: The Politics of Global Governance ». Sydney Law Review, vol. 27, p. 1-24.

Kindornay, Shannon, L'Institut Nord-Sud (INS), Fraser Reilly-King et Conseil canadien pour la coopération internationale. 2013. Investir dans les affaires du développement: Les approches des bailleurs de fonds bilatéraux dans le travail avec le secteur privé. Conseil canadien pour la coopération internationale ( C C C I ). En ligne.

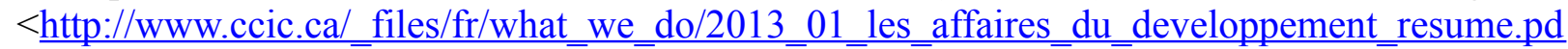
f>.

Kingfisher, C. et J. Maskovsky. 2008. «Introduction: The Limits of Neoliberalism » . Critique of Anthropology, vol. 28, no 2, p. 115-126.

Kothari, Uma. 2005. « Authority and Expertise: The Professionalisation of International Development and the Ordering of Dissent ». Antipode, vol. 37, no 3, p. 425-446.

Lane, Charles R. 1994. « Pastures lost: alienation of Barabaig land in the context of land policy and legislation in Tanzania ». Nomadic Peoples, p. 81-94.

Lane, Charles R et Jules N Pretty. 1990. Displaced pastoralists and transferred wheat technology in Tanzania. Coll. «Gatekeeper series ». Londres : International Institute for Environnement and

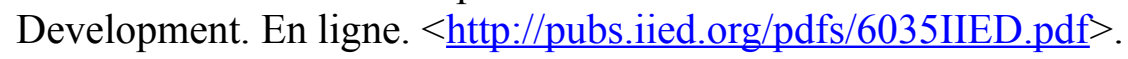


Larner, Wendy. 2000. «Neo-liberalism: Policy, ideology, governmentality ». Studies in political economy, vol. 63.

Latouche, Serge. 1986. Faut-il réfuser le développement?: essai sur l'anti-économique du tiers monde. Presses universitaires de France.

Laval, Christian et Pierre Dardot. 2009. La nouvelle raison du monde. Essai sur la société néolibérale. Paris, France : La Découverte.

Lave, Rebecca. 2012. «Neoliberalism and the Production of Environmental Knowledge ». Environment and Society, vol. 3, no 1. En ligne. <http://berghahnjournals.com/view/journals/environment-and-society/3/1/air-es030103.xml>. Consulté le 31 juillet 2016.

Leclerc, Jean-Claude. 2013. «Feu l'ACDI - Ces pauvres qu'Ottawa veut sortir de la misère ». Le Devoir, 25 mars 2013, sect. Société, Éthique et religion. En ligne. < $\underline{\text { http://www.ledevoir.com/so- }}$ ciete/ethique-et-religion/374065/ces-pauvres-qu-ottawa-veut-sortir-de-la-misere $>$.

Legouté, Jean-Ronald. 2001. «Définir le développement: historique et dimension d'un concept plurivoque ». Économie politique internationale, vol. 1, no 1. Coll. « Groupe de recherche sur l'intégration continentale de l'Université du Québec à Montréal », p. 1-43.

Le Naëlou, Anne. 1994. «L'expertise à l'œuvre : le cas de la coopération européenne en Bolivie ». Bulletin de l'APAD, vol. 8, p. 1-12.

Li, Tania Murray. 2007. The Will to Improve: Governmentality, Development, and the Practice of Politics. Duke University Press.

Li, Tanya Murray. 2005. "Beyond "the State" and Failed Schemes ». American Anthropologist, vol. 107, no 3, p. 383-394.

Lukács, Georg. 1960. Histoire et conscience de classe: essais de dialectique marxiste. Traduction par K. Axelos et J. Bois, Paris : Éditions de minuit.

Mackrael, Kim. 2012. « Ottawa signals shift in foreign-aid policy toward private sector ». The Globe and Mail, 23 novembre 2012. En ligne. $<\underline{\text { http://www.theglobeandmail.com/news/politics/otta- }}$ wa-signals-shift-in-foreign-aid-policy-toward-private-sector/article5582948/>. Consulté le 19 mars 2015.

Malouin, Eryck. 2002. Éthique de la recherche social; Consentement libre et éclairé, confidentialité et vie privée. Orientations. Fonds québécois de recherche sur la société et la culture. En ligne.

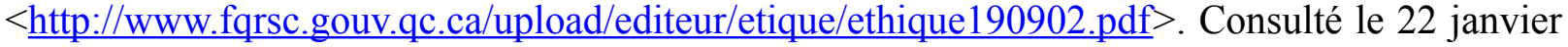
2014.

Marx, Karl. 1976. Le capital: critique de l'économie politique. Livre I. Trad. par Joseph Roy. Paris : Ed. Sociales. 
Mehta, Lyla. 2001. « The World Bank and its emerging knowledge empire ». Human Organization, vol. 60 , no 2 , p. 189-196.

MiningWatch Canada. 2013. « Mining and development - how much will it cost us to clothe the naked emperor? » En ligne. < http://www.miningwatch.ca/fr/node/7203 >. Consulté le 19 mars 2015.

Ministère des Affaires étrangères, du commerce et du développement. 2009. " Une nouvelle approche efficace pour l'aide canadienne: Notes pour une allocution prononcée par l'honorable Beverley J. Oda ministre de la coopération internationale au Centre Munk d'études internationales ». En

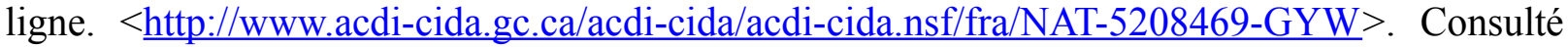
le 31 octobre 2015.

2015. «Aide et foire aux questions (FAQ)». Banque de projet de développement international. En ligne. < http://www.acdi-cida.gc.ca/cidaweb/cpo.nsf/vLUWebHelpFr/AideRecherche> . Consulté le 2 septembre 2015.

Mitchell, Timothy. 2002. Rule of experts: Egypt, techno-politics, modernity. Berkeley, CA : Univ of California Press.

Montane, M.-A. 2002. «Paroles de leaders: L'entretien semi-directif de recherche est-il adaptable à de nouvelles situations d'enquête? » Dans Méthodes et outils des sciences sociales. Innovation et renouvellement, sous la dir. de P. Blanchard et T. Ribémont, p. 19-33. Paris : L'Harmattan.

Morin, David et Stéphane Roussel. 2014. «Autopsie de la politique étrangère de Stephen Harper: un examen préliminaire ». Canadian Foreign Policy Journal, vol. 20, no 1, p. 1-8.

Mosse, David. 2005. Cultivating development an ethnography of aid policy and practice. Coll. «Anthropology, culture and society ». London; Ann Arbor, MI : Pluto Press.

Niane, Boubacar. 2011. «Experts vs chercheurs dans la fabrication et la circulation des savoirs et sens sociopolitiques au Sénégal ». Cahiers d'études africaines, vol. 2, no 202-203, p. 549-562.

North, Douglas. 2005. Le processus du développement économique. Paris, France: Éditions d'Organisations.

OECD. 2014. «Canada». Dans Development Co-operation Report 2014, par OECD, p. 280-283. OECD Publishing. En ligne. < $\underline{\text { http://www.oecd-ilibrary.org/development/development-co- }}$ operation-report-2014/canada_dcr-2014-31-en>. Consulté le 25 mars 2015.

2015. Development Co-operation Report 2015. Coll. « Development Co-operation Report ».

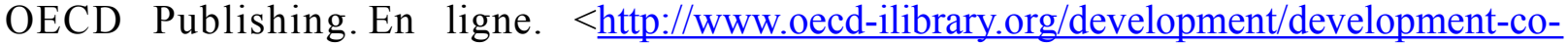
operation-report-2015 dcr-2015-en>. Consulté le 22 septembre 2016.

Ong, Aihwa. 2007. « Neoliberalism as a mobile technology ». Transactions of the Institute of British Geographers, vol. 32, no 1, p. 3-8. 
Organisation de coopération et de développement économique (OCDE). 2001. Le déliement de l'aide au pays les moins avancés. Synthèse. Coll. «L'Observateur - OCDE ». Paris, France : Organisation de coopération et de développement économique (OCDE). En ligne. $<$ http://www.oecd.org/fr/finances/35919775.pdf $>$.

Ovaska, Tomi. 2003. « The Failure of Development Aid. » Cato Journal, vol. 23, no 2, p. 175-188.

Owakah, Francis E. et Robert D. Aswani. 2009. « Technocracy and Democracy : The Challenges to Development in Africa ». Thought and Practice, vol. 1, no 1, p. 87-99.

Paquette, Francis. 2014. « La confessionnalisation de l'aide canadienne au développement ». Mémoire, Montréal, Québec : Université de Montréal.

Parpart, Jane L. 2003. "Deconstructing the development expert: Gender, development and the "vulnerable groups" ». Dans Feminism/postmodernism/development, p. 221-243. London; New York: Routledge. E n lign e. <http://search.ebscohost.com/login.aspx? direct $=$ true $\&$ scope $=$ site $\& d b=$ nlebk $\& d b=$ nlabk\&AN=94873 $>$. Consulté le 17 décembre 2014.

Parti Libéral du Canada. 2015. « Aider les plus pauvres ». En ligne. $<\underline{\text { https://www.liberal.ca/fr/change- }}$

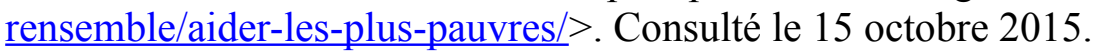

Peck, Jamie et Adam Tickell. 2002. « Neoliberalizing Space ». Antipode, vol. 34, no 3, p. 380-404.

Prevost, Benoît. 2005. «Les fondements philosophiques et idéologiques du nouveau discours sur le développement ». Economies et Sociétés, no 3, p. 477-496.

Rayner, Steve. 2003a. "Who's in Charge? Worldwide Displacement of Democratic Judgment by Expert Assessments ». Economic and Political Weekly, vol. 38, no 48, p. 5113-5119.

. 2003b. «Democracy in the age of assessment: reflections on the roles of expertise and democracy in public-sector decision making ». Science and Public Policy, vol. 30, no 3, p. $163-170$.

Reynolds, Martin. 2008. «Reframing expert support for development management ». Journal of International Development, vol. 20, no 6, p. 768-782.

Richland, Justin B. 2009. «On neoliberalism and other social diseases: The 2008 sociocultural anthropology year in review ». American Anthropologist, vol. 111, no 2, p. 170-176.

Rip, Arie. 2003. « Constructing expertise in a third wave of science studies? » Social studies of science, vol. 33, no 3, p. 419-434.

Rist, Gilbert. 2007. Le développement: histoire d'une croyance occidentale. Paris : Presses de Sciences po.

Roediger, David R. 2002. Colored white: Transcending the racial past. Vol. 10. Univ of California Press. 
Roethlisberger, F.J. et William J. Dickson. 1943. Management and the worker. Cambridge, Massachussetts : Harvard University Press.

Sachs, Jeffrey. 2005. The End of Poverty: Economic Possibilities for Our Time. New York : Penguin Press.

Said, Edward W. 1979. Orientalism. 1st Vintage Books ed. New York : Vintage Books.

Samantha Nutt. 2012. "Should NGOs take the corporate bait? » The new humanitarians. Globe and Mail. En ligne. <http://www.theglobeandmail.com/globe-debate/should-ngos-take-thecorporate-bait/article1359759/>. Consulté le 27 janvier 2012.

Sandbrook, Richard. 2007. « Alternatives to neo-liberalism in the third world ». RENEWAL-LONDON-, vol. 15 , no $2 / 3$, p. 46.

Savoie-Zajc, Lorraine. 2007. «Comment peut-on construire un échantillonnage scientifiquement valide? » Recherches qualitatives, no 5. Coll. « Hors-Série », p. 99-111.

2009. «L'entrevue semi-dirigée ». Dans Recherche sociale: de la problématique à la collecte des données, sous la dir. de Benoît Gauthier, p. 337-360. Québec : Presses de l’Université du Québec.

Scott, James C. 1998. Seeing like a state: how certain schemes to improve the human condition have failed. New Haven, Conn. [u.a.] : Yale Univ. Press.

Simon, Herbert A. 1991. « Bounded Rationality and Organizational Learning ». Organization Science, vol. 2, no 1, p. 125-134.

Smith, Andy. 2000. «Comment le néolibéralisme gagne sur le territoire. À propos de certaines transformations récentes du rugby ». Politix, vol. 13, no 50, p. 73-92.

Sogge, David. 2003. Les mirages de l'aide internationale: quand le calcul l'emporte sur la solidarité. Trad. par Danielle Collignon et Marie-Claude Rochon. Paris; Tunis; Lausanne : Editions de l'Atelier; Cérès éditions ; Editions d'En bas [etc.].

Stackhouse, John. 2001. Out of poverty: and into something more comfortable. Toronto : Vintage Canada.

St. Clair, Asuncion Lera. 2006. « The World Bank as a Transnational Expertised Institution ». Global Governance, vol. 12, no 1, p. 77-95.

Stirrat, R. L. 2000. « Cultures of Consultancy ». Critique of Anthropology, vol. 20, no 1, p. 31-46.

Subramaniam, Mangala. 2007. «NGOs and Resources in the Construction of Intellectual Realms: Cases from India ». Critical Sociology, vol. 33, no 3, p. 551-573. 
Swidler, Ann et Susan Cotts Watkins. 2009. " "Teach a Man to Fish": The Sustainability Doctrine and Its Social Consequences ». World Development, vol. 37, no 7, p. 1182-1196.

Teichman, Judith. 1997. «Economic Reform and Technocratic Decision Making ». Studies in Comparative international Development, vol. 32, no 1, p. 31-55.

Van Rooy, Alison. 1998. Civil society and the aid industry. London, Angleterre : Earthscan.

Wacquant, Loïc. 2010. «L'habitus comme objet et méthode d'investigation: Retour sur la fabrique du boxeur ». Actes de la recherche en sciences sociales, vol. 184, no 4, p. 108.

- 2012. «Three steps to a historical anthropology of actually existing neoliberalism ». Social Anthropology, vol. 20, no 1, p. 66-79.

Wade, Robert. 1996. "'Japan, the World Bank, and the Art of Paradigm Maintenance: The East Asian Miracle in Political Perspective' ». New Left Review, vol. 217, p. 3-38.

Watkins, Susan Cotts et Ann Swidler. 2013. "Working Misunderstandings: Donors, Brokers, and Villagers in Africa's AIDS Industry ».Population and Development Review, vol. 38, p. 197-218.

Wilks, Alex et Fabien Lefrançois. 2002. Blinding with Science or Encouraging Debate? How World Bank Analysis Determines PRSP Policies. Bretton Woods Projects.

Williamson, Claudia R. 2009. "Exploring the failure of foreign aid: The role of incentives and information ». The Review of Austrian Economics, vol. 23, no 1, p. 17-33.

Williamson, John. 1990. "What Washington means by policy reform ». Dans Latin American adjustment: How much has happened, sous la dir. de John Williamson, p. 7-20. Institute for International Economics.

Wilson, Gordon. 2006. « Beyond the Technocrat? The Professional Expert in Development Practice ». Development and Change, vol. 37, no 3, p. 501-523.

Ziai, Aram. 2013. "The discourse of "development" and why the concept should be abandoned ». Developpment in Practice, vol. 23, no 1, p. 123-136. 
ANNEXE 1 - COPIE DU TEXTE DU PREMIER CONTACT TÉLÉPHONIQUE 
Bonjour, mon nom est Laurent Paradis-Charette. Je suis étudiant à la maîtrise en sciences sociales du développement territorial et je mène actuellement une recherche sur le thème de l'utilisation de l'expertise dans les agences d'aide en développement international dans le cadre de l'écriture de mon mémoire de maîtrise nécessaire en vue d'obtenir le diplôme. Accepteriez-vous de participer à une entrevue de 30 minutes à une heure à ce sujet, à un lieu et à un moment de votre choix? 
ANNEXE 2 - COPIE DU TEXTE DU PREMIER CONTACT POSTAL OU PAR COURRIER ÉLECTRONIQUE 
Bonjour,

Je vous contacte, car je mène actuellement des recherches sur l'utilisation de l'expertise pour le développement international dans les agences d'aide au développement dans le cadre de mon projet de mémoire de maitrise en sciences sociales du développement à l'Université du Québec en Outaouais.

J'aimerais en apprendre plus sur les problèmes et les succès rencontrés par les économistes dans la production d'avis pour les agences de développements.

Je crois que votre expérience pourrait contribuer à mieux comprendre certains enjeux de la production des avis dans les agences de développement international. À cet effet, je souhaite vous rencontrer pour discuter de ces aspects et recueillir votre opinion dans le cadre d'un entretien de recherche sur l'utilisation de l'expertise économique pour le développement dans les agences d'aide au développement.

Si vous êtes disponible, je pourrais me déplacer à un lieu et à un moment de votre convenance. La rencontre pourrait aussi avoir lieu à l'Université du Québec en Outaouais située à Gatineau.

Je vous remercie à l'avance de votre intérêt pour mes recherches.

Sincèrement,

Laurent Paradis-Charette

Étudiant à la maîtrise en sciences sociales du développement territorial

Département des sciences sociales

Université du Québec en Outaouais

Gatineau, Québec

parl08(a)uqo.ca

819 595-3900 (XXXX) $)^{23}$

23 Nous avons supprimé les numéros de poste de l'annexe, car ils peuvent être utilisés par un autre employé de l'UQO sans lien avec cette recherche. 
ANNEXE 3 - GUIDE D'ENTRETIEN 
Quelles études avez-vous?

\section{Quelques questions sur vous;}

Votre parcours professionnel?

De quoi êtes-vous spécialiste?

Quelles sont vos compétences particulières?

\section{Parlons des experts;}

1. Pouvez-vous nous dire selon vous, ce qu'est un expert?

2. Où trouve-t-on des experts?

3. Que pensez-vous de l'utilisation du terme expert?

1. par les médias?

2. par le gouvernement?

3. par les ONG?

4. par le secteur privé?

\section{Parlons de votre travail pour l'agence;}

1. Pouvez-vous nous en dire plus à propos de votre rôle et de vos fonctions pour l'agence/l'organisme?

1. Pour quelles raisons on fait appel à vous?

2. Avec quels types d'expert vous interagissez?

1. Comment se passent les interactions?

3. Est-ce que vous pourriez me décrire une situation où vous vous êtes senti « expert »?

1. Comment ce que vous faites se traduit sur le terrain?

4. Dans le cadre de votre travail si vous estimez devoir faire des recommandations, comment procédez-vous?

5. Que pensez-vous de l'utilisation de vos recommandations?

\section{Pour terminer, parlons de l'aide au développement}

1. Selon vous, comment un expert économique peut-il contribuer à améliorer la situation de pauvreté dans le monde?

1. Certains pourraient dire que les experts peuvent nuire au développement, qu'en pensezvous? 
ANNEXE 4 - Formulaire DE CONSENTEMENT 
Case postale 1250, succursale B, Gatineau (Québec), Canada J8X 3X7 Téléphone (819) 595-3900

www.uqo.ca

\section{Formulaire de consentement}

Titre du projet :

L'utilisation de l'expertise en développement international dans les agences d'aide au développement au Canada : le point de vue de experts.

Chercheur principal : Laurent Paradis-Charette

Directeur de recherche: Dimitri della Faille

Département : $\quad$ Sciences sociales, Université du Québec en Outaouais

Par la présente, nous sollicitons votre participation à la recherche en titre qui vise à mieux comprendre les défis contemporains de l'expertise économique pour le développement. Dans cet entretien, il sera question de recueillir votre opinion, vos impressions ainsi que votre expérience à propos de l'utilisation de l'expertise économique pour le développement international dans les agences d'aide au développement.

Votre seule participation à ce projet de recherche consiste à être questionné pendant environ une heure sur le thème indiqué ci-dessus. Au début de cet entretien, le chercheur vous présentera les objectifs de la recherche et les thèmes des questions. Au cours de l'entretien, le chercheur vous posera des questions générales en fonction de ses objectifs. Au besoin, il posera des questions plus spécifiques de précision. Les données recueillies sont anonymes, c'est-à-dire que nous n'identifierons pas dans les rapports de recherche, articles scientifiques, autres publications et communications scientifiques le nom de personnes ayant fourni les informations. De plus, le cas échéant, nous n'indiquerons qu'en terme générique (très larges) le type d'organisation à laquelle vous appartenez et votre région du monde. Cela dit, nous ne sommes pas en mesure de garantir totalement l'anonymat. Il serait en effet possible que certains lecteurs de ces publications puissent vous reconnaître au travers des propos qui seraient rapportés de manière anonyme dans les résultats de recherche.

Les données recueillies dans ce projet seront conservées dans l'ordinateur du chercheur ainsi que dans un disque dur de sauvegarde qui sont protégés par un mot de passe dans un bureau fermé à clé auquel seul le chercheur a accès. Elles seront détruites cinq ans après la fin de la recherche. Elles ne seront pas utilisées à d'autres fins que celle décrite dans le présent document, à moins que vous n'y consentiez.

Votre participation à cette étude se fait sur une base volontaire. Nous sollicitons votre participation, car sans elle, nous ne pourrons pas obtenir les informations indispensables pour la réalisation de cette recherche. Vous êtes entièrement libre de participer ou non, et de vous retirer en tout temps de la recherche sans préjudice. Dans ce cas, les données recueillies seront détruites immédiatement. Vous pourrez choisir de ne pas répondre à certaines questions sans que cela ait une incidence sur votre participation. Vous pouvez aussi, une fois l'entrevue terminée, demander à ce que les réponses fournies à l'une ou plusieurs des questions soient retirées des notes du chercheur.

Les risques associés à votre participation sont nuls. En tant que chercheur, nous nous engageons à mettre en œuvre tous les moyens nécessaires pour nulifier les risques en rendant toutes les données anonymes lors de l'analyse et la présentation des résultats. Jamais votre nom, ni l'intitulé de votre poste, ni votre pays d'origine, ni votre organisme ne figureront dans les documents d'analyse et dans les résultats. Soyez assurés que toutes les opinions exprimées durant notre rencontre restent confidentielles et anonymes. Aucune compensation d'ordre monétaire n'est accordée. 
Si vous avez des questions concernant les aspects éthiques de ce projet de recherche, veuillez communiquer avec $M$. André Durivage au 1-819-595-3900, poste 1781, (andre.durivage@,uqo.ca) président du comité d'éthique de la recherche de l'Université du Québec en Outaouais.

Votre signature atteste que vous avez clairement compris les renseignements concernant votre participation au projet de recherche et indique que vous acceptez d'y participer. Elle ne signifie pas que vous acceptez d'aliéner vos droits et de libérer le chercheur ou les responsables de leurs responsabilités juridiques ou professionnelles. Votre participation devant être aussi éclairée que votre décision initiale de participer au projet, vous devez en connaître tous les tenants et aboutissants au cours du déroulement de la recherche. En conséquence, vous ne devez jamais hésiter à demander des éclaircissements ou de nouveaux renseignements au cours du projet.

Veuillez encerclez le choix approprié :

J'accepte que le chercheur prenne des notes

Je préfère que le chercheur ne prenne pas de notes

Avec votre permission, nous aimerions pouvoir conserver les données recueillies à la fin du présent projet pour d'autres activités de recherche dans le même domaine pour lequel vous êtes aujourd'hui invités à participer. Afin de préserver vos données personnelles et votre identité, les données seront anonymisées, c'est-à-dire qu'il ne sera plus possible à quiconque de pouvoir les relier à votre identité. Nous nous engageons à respecter les mêmes règles d'éthique que pour le présent projet.

Il n'est pas nécessaire de consentir à ce volet pour participer à la présente recherche. Si vous refusez, vos données seront détruites à la fin du présent projet. Si vous acceptez, vos données seront conservées pour une période de 5 ans après la fin du présent projet et ensuite détruites.

\section{Veuillez encerclez le choix approprié :}

$\checkmark$ J'accepte une utilisation secondaire des données que je vais fournir

$\checkmark \quad$ Je refuse une utilisation secondaire des données que je vais fournir

Après avoir pris connaissance des renseignements concernant ma participation à ce projet de recherche, j'appose ma signature signifiant que j'accepte librement d'y participer dans les conditions précisées ci-dessus. Le formulaire est signé en deux exemplaires et j'en conserve une copie.

Nom du participant :

Signature du participant :

Date :

Nom du chercheur :

Signature du chercheur :

Date :

Voici les coordonnées du chercheur et du directeur de la recherche pour le/les rejoindre au besoin :

Laurent Paradis-Charette

Étudiant à la maîtrise Département des Sciences sociales

Université du Québec en Outaouais succursale B

Gatineau (Québec), Canada J8X 3X7

Téléphone : (819) 595-3900 poste XXXX

Courriel : parl08(a)uqo.ca
Dimitri della Faille

Professeur

Département des sciences sociales

Université du Québec en Outaouais succursale B

Gatineau (Québec), Canada J8X 3X7

Téléphone : (819) 595-3900 poste XXXX

Courriel : dimitri.dellafaille(a)uqo.ca 